\title{
LiU-ITN-TEK-A--13/047--SE
}

\section{Improving information perception from digital images for users with dichromatic color vision \\ Omid Shayeghpour}

2013-09-23

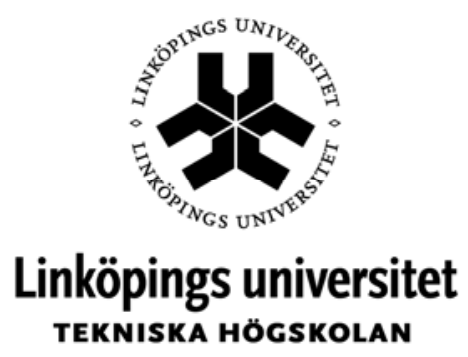




$$
\text { LiU-ITN-TEK-A--13/047--SE }
$$

\title{
Improving information perception from digital images for users with dichromatic color vision
}

Examensarbete utfört i Medieteknik vid Tekniska högskolan vid Linköpings universitet

\section{Omid Shayeghpour}

\author{
Handledare Sasan Gooran
}

Examinator Daniel Nyström

Norrköping 2013-09-23 


\section{Upphovsrätt}

Detta dokument hålls tillgängligt på Internet - eller dess framtida ersättare under en längre tid från publiceringsdatum under förutsättning att inga extraordinära omständigheter uppstår.

Tillgång till dokumentet innebär tillstånd för var och en att läsa, ladda ner, skriva ut enstaka kopior för enskilt bruk och att använda det oförändrat för ickekommersiell forskning och för undervisning. Överföring av upphovsrätten vid en senare tidpunkt kan inte upphäva detta tillstånd. All annan användning av dokumentet kräver upphovsmannens medgivande. För att garantera äktheten, säkerheten och tillgängligheten finns det lösningar av teknisk och administrativ art.

Upphovsmannens ideella rätt innefattar rätt att bli nämnd som upphovsman i den omfattning som god sed kräver vid användning av dokumentet på ovan beskrivna sätt samt skydd mot att dokumentet ändras eller presenteras i sådan form eller i sådant sammanhang som är kränkande för upphovsmannens litterära eller konstnärliga anseende eller egenart.

För ytterligare information om Linköping University Electronic Press se förlagets hemsida http://www.ep.liu.se/

\section{Copyright}

The publishers will keep this document online on the Internet - or its possible replacement - for a considerable time from the date of publication barring exceptional circumstances.

The online availability of the document implies a permanent permission for anyone to read, to download, to print out single copies for your own use and to use it unchanged for any non-commercial research and educational purpose. Subsequent transfers of copyright cannot revoke this permission. All other uses of the document are conditional on the consent of the copyright owner. The publisher has taken technical and administrative measures to assure authenticity, security and accessibility.

According to intellectual property law the author has the right to be mentioned when his/her work is accessed as described above and to be protected against infringement.

For additional information about the Linköping University Electronic Press and its procedures for publication and for assurance of document integrity, please refer to its WWW home page: http://www.ep.liu.se/ 


\section{Abstract}

Color vision deficiency (CVD) is the inability or limited ability to recognize colors and discriminate between them. A person with this condition perceives a narrower range of colors compared to a person with a normal color vision. A growing number of researchers are striving to improve the quality of life for CVD patients. Finding cure, making rectification equipment, providing simulation tools and applying color transformation methods are among the efforts being made by researchers in this field.

In this study we concentrate on recoloring digital images in such a way that users with CVD, especially dichromats, perceive more details from the recolored images compared to the original image. The main focus is to give the CVD user a chance to find information within the picture which they could not perceive before. However, this transformed image might look strange or unnatural to users with normal color vision.

During this color transformation process, the goal is to keep the overall contrast of the image constant while adjusting the colors that might cause confusion for the CVD user. First, each pixel in the RGB-image is converted to HSV color space in order to be able to control hue, saturation and intensity for each pixel and then safe and problematic hue ranges need to be found. The method for recognizing these ranges was inspired by a condition called "unilateral dichromacy" in which the patient has normal color vision in one eye and dichromacy in another. A special grid-like color card is designed, having constant saturation and intensity over the entire image, while the hue smoothly changes from one block to another to cover the entire hue range.

The next step is to simulate the way this color card is perceived by a dichromatic user and finally to find the colors that are perceived identically from two images and the ones that differ too much. This part makes our method highly customizable and we can apply it to other types of CVD, even personalize it for the color vision of a specific observer. The resulting problematic colors need to be dealt with by shifting the hue or saturation based on some pre-defined rules.

The results for the method have been evaluated both objectively and subjectively. First, we simulated a set of images as they would be perceived by a dichromat and compared them with simulated view of our transformed images. The results clearly show that our recolored images can eliminate a lot of confusion from user and convey more details. Moreover, an online questionnaire was created and 39 users with CVD confirmed that the transformed images allow them to perceive more information compared to the original images. 


\section{Introduction}

Color vision deficient users have problem discriminating different colors. In chapter 1, we will introduce CVD and discuss its causes and types and how to diagnose such conditions. The project objectives as well as our goals for this thesis will be presented in this chapter as well.

Chapter 2 will be dedicated to the basic information about digital color imaging. This chapter begins with the introduction to the different color spaces that will be required in the rest of this thesis and concludes by explaining how a typical simulation tool, simulates the vision of a color vision deficient user.

In Chapter 3, our main method will be explained in details. First, a brief background of different efforts done in the field of CVD will be presented. Throughout the rest of this chapter we will explain in detail our goals and how we try to achieve them in this thesis. Various test images will be utilized in this chapter which somewhat cover some part of our results as well.

In Chapter 4, we will discuss a few other distinct approaches that other researchers introduced in order to tackle problems of similar manner. We will also try to show some parts of their result sets to generate an understanding about possible differences between these approaches and our proposed method.

We have tested our method in two ways: first, we compared the dichromatic simulation of the original image with the simulation of our transformed image and evaluated if our method improved the information perception or not. This type of evaluation was done throughout Chapters 3 and 4 while the basics of how such a simulation takes place was presented in Chapter 2.

Second, we tried to validate our work by testing the results through actual color vision deficient patients. Several pictures were transformed by our method and through a questionnaire we asked different questions about those pictures before and after transformation. Those results will be presented in Chapter 5. We will present a suggestion for the future work in this field by combining our method with the personalized simulation that will also be explained in Chapter 5 . A short summary of our work will conclude this chapter. 


\section{Table of Contents}

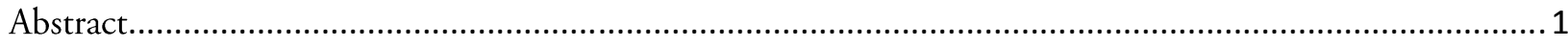

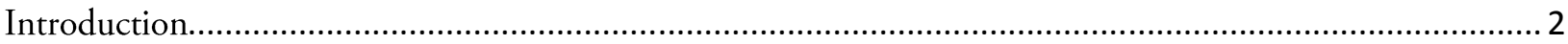

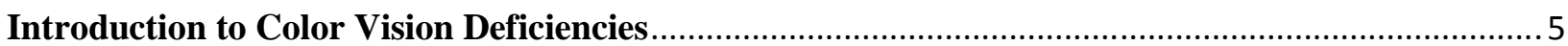

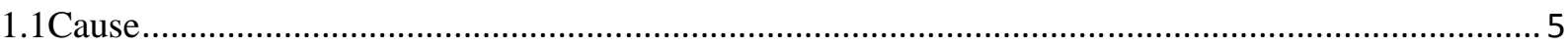

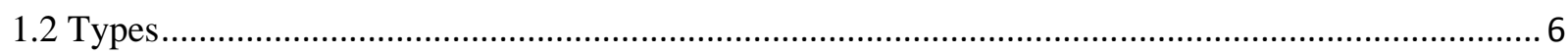

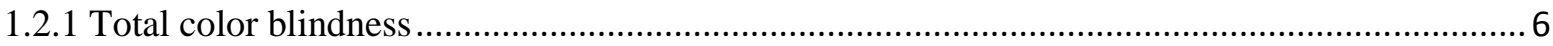

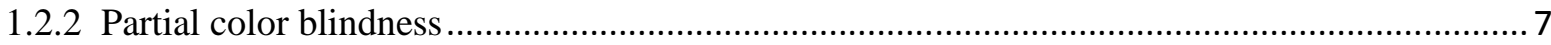

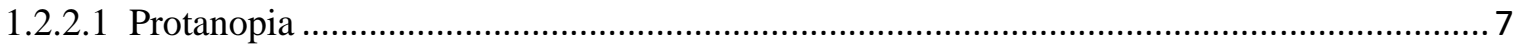

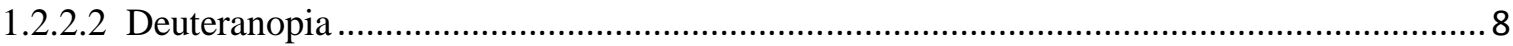

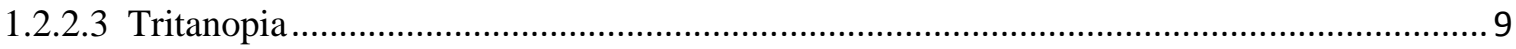

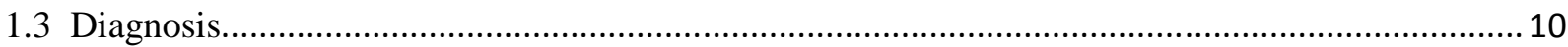

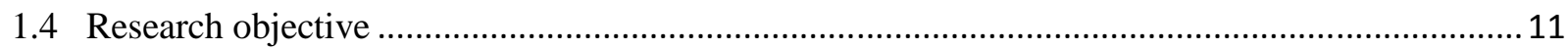

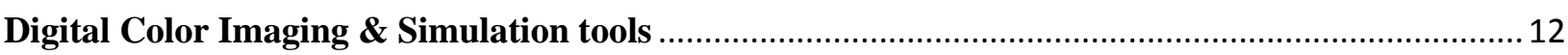

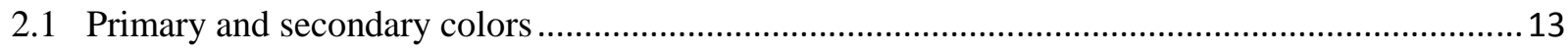

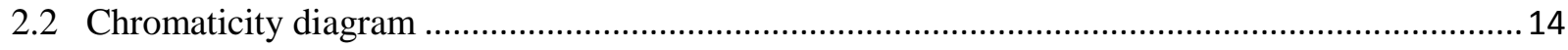

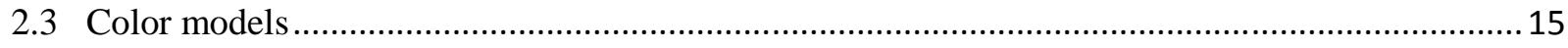

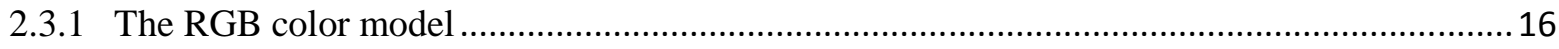

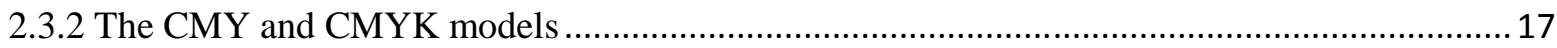

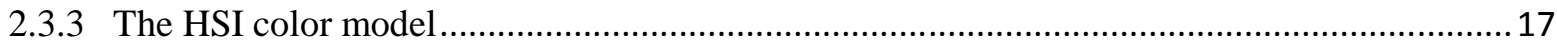

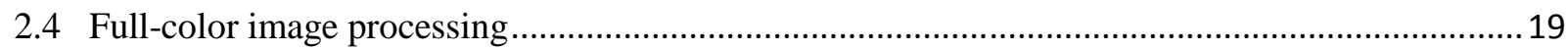

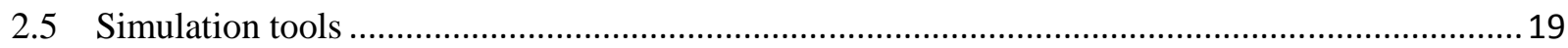

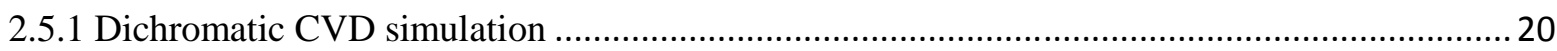

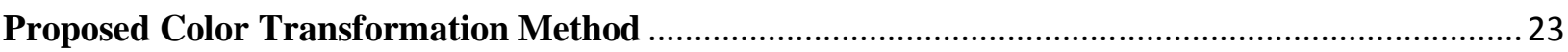

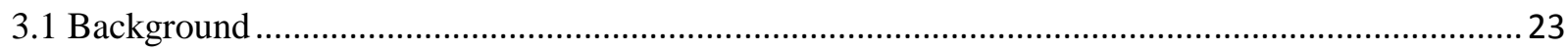

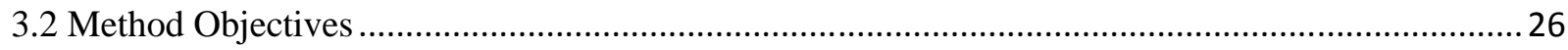

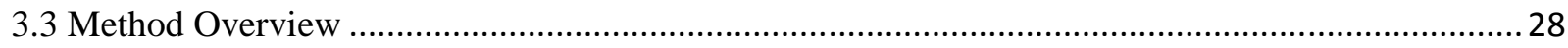

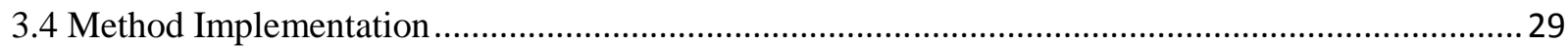

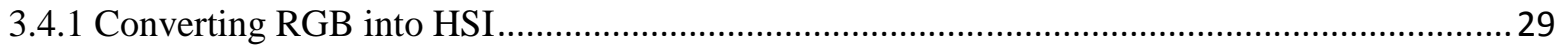




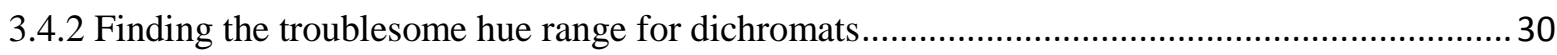

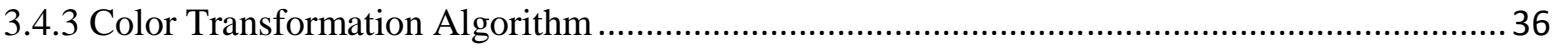

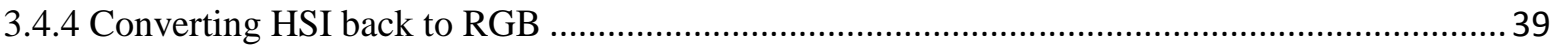

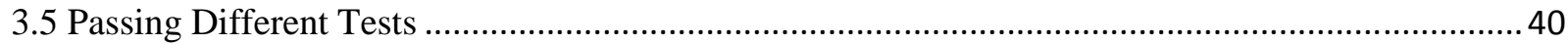

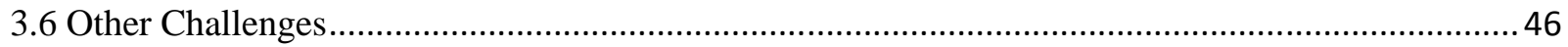

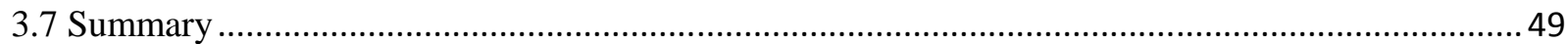

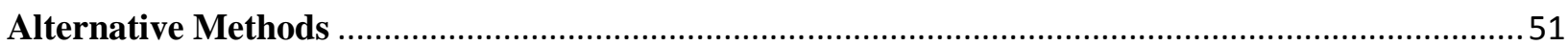

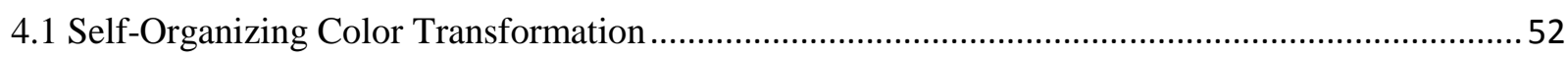

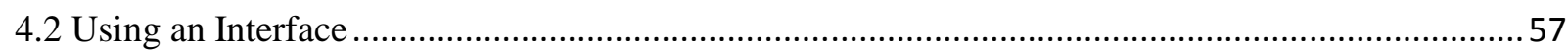

4.3 Recoloring Images using Gray-scale Conversion ....................................................................... 58

4.4 Information Preserving Color Transformation............................................................................... 62

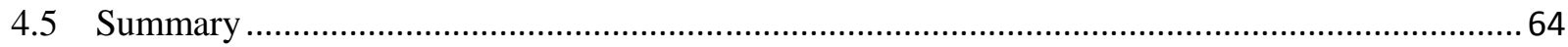

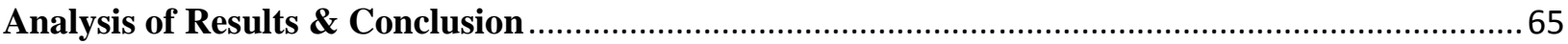

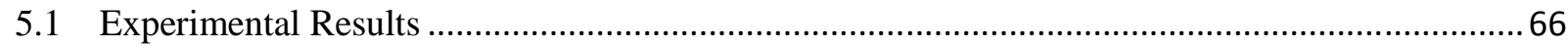

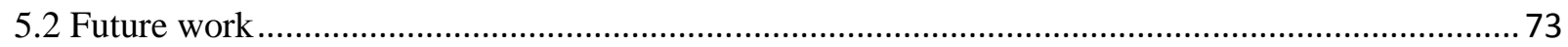

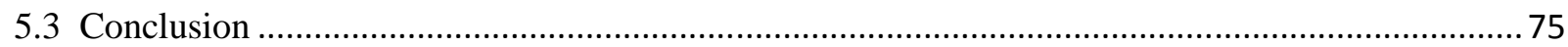

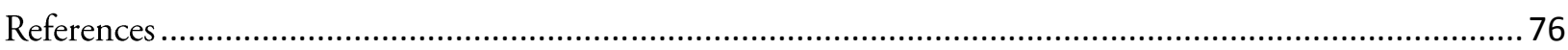




\section{Chapter 1}

\section{Introduction to Color Vision Deficiencies}

Contrary to what most people think, being color blind does not necessarily mean they cannot see any color. The more accurate term to use would be "Color Vision Deficiencies" to eliminate this confusion. Color vision deficiency (CVD) is the inability or decreased ability to perceive colors or discriminate between different colors that can easily be distinguished by most other people under normal lighting situations. Simply put, a person suffering from CVD perceives a narrower color spectrum compared to an individual with normal color vision $[1,2]$.

\subsection{Cause}

The retina of the human eye contains about 7 million cone cells ${ }^{1}$ and more than 100 million rod cells $^{2}$ that together enable normal vision. For observers with normal color vision, there are three distinct kinds of cones; some with peak sensitivity lying in the short wavelengths regions of the spectrum (S-cone often accounted for blue), some in the medium wavelengths (M-cone for green) and some in the longer wavelengths (L-cone for red) ${ }^{3}$. Different colors are perceived when the different types of cones are stimulated to different degrees [3]. Pigments have the ability to absorb some colors and reflect others. For instance, if an object appears green to us, it means that all other color wavelengths except green are absorbed. The most usual cause of having CVD is absence or abnormality of one or more sets of these cone types [1,4-7].

Color vision deficiency can be acquired as a result of physical or chemical damage to the eye, the optic nerve or parts of the brain ${ }^{4}$. This, however, is not the reason in most cases as CVD can also

\footnotetext{
${ }^{1}$ Cones are the receptors highly sensitive to color. Cone vision is called photopic or bright-light vision [3].

${ }^{2}$ Rods give a general, overall picture of the field of view. They are not involved in color vision and are sensitive to low levels of illumination. Rod vision is known as scotopic or dim-light vision [3].

${ }^{3}$ This means a normal or correct color vision should be trichromatic, meaning that colors can be represented in terms of quantities of three color wavelengths. Color theory is discussed in more detail in Chapter 2.

${ }^{4}$ CVD can also be acquired as a result of accidents or diseases like Diabetes, Cataract, etc. [2, 4].
} 
be inherited as a genetic disorder. The genes that produce photo pigments are carried on the Xchromosome $^{1}$. If some of these genes are missing or damaged it can lead to some sort of color vision deficiency. Males only have one X-chromosome; hence males are more susceptible to CVD than females in case of defect in genes. On the other hand, since females possess two Xchromosome, a functional gene on either one of them would suffice to give them the normal color vision $[4,8]$. Although some types of color vision deficiencies are not gender-related, this difference in male and female genetic system which is the reason for the most common CVD types, result in larger population of males struggling with CVD in comparison to females. Researches show that about $8 \%$ of males and $0.5 \%$ of females worldwide suffer from color vision deficiency among which a quarter are affected by dichromacy- one of the most common color vision defects that has a genetic origin [5].

\subsection{Types}

We can categorize color vision deficiencies into two major types, both of which have a direct relation to the unavailable or faulty photoreceptors in the eye:

\subsubsection{Total color blindness}

This type of color vision deficiencies is called Monochromacy. The people with this type of deficiency lack the ability to distinguish colors altogether. Hence, such people view everything as if they are watching a black and white television set. Monochromacy occurs when two or all three of the cone pigments are missing or defective [8]. Figure 1.1 shows an example of how a monochromatic person sees a red apple. This type of CVD is extremely rare and will not be a part of the research done in this thesis, simply because there is nothing to be done to improve color discrimination when no color can be perceived in the first place.
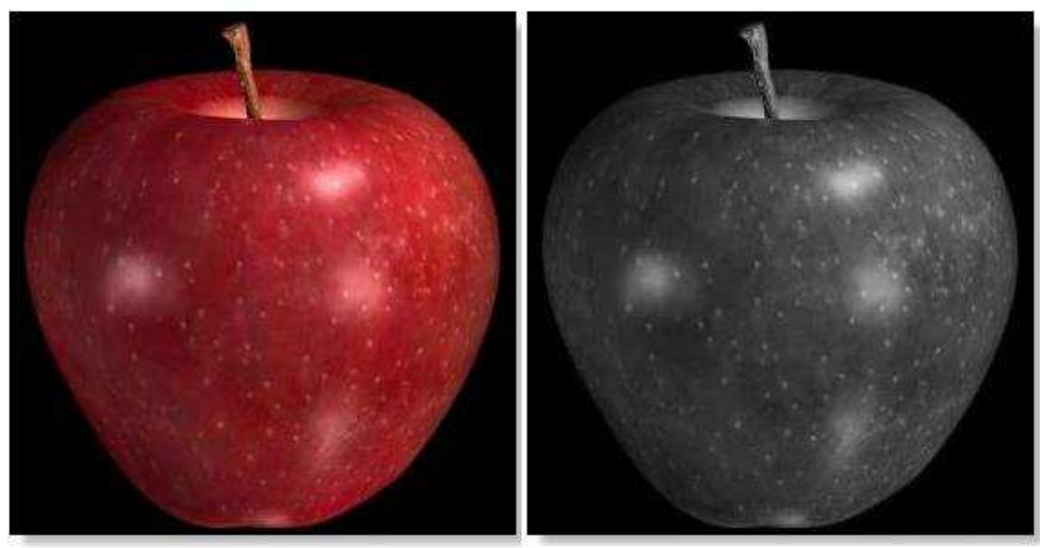

Figure 1.1: (left) The original picture (right) The simulated monochromatic vision

\footnotetext{
${ }^{1}$ One of the 46 structures in the human cell nucleus which carries genes that contain the hereditary material controlling the growth and characteristics of the body [4].
} 


\subsubsection{Partial color blindness}

This type of CVD is categorized into two main groups itself. First group is called Dichromacy. It is a severe case of CVD that results when one of the three cone types is missing [9]. A person who is dichromatic has difficulty discriminating between many spectral hues [4]. The other group is called Anomalous Trichromacy. People with this type of deficiency have the ability to visualize most colors, but abnormally. All three cone types are available but the peak of sensitivity is shifted for one of them, which results in a smaller color spectrum and also perceiving some colors abnormally $[1,4]$.

Each one of these two groups is categorized into three subcategories based on the missing or malfunctioning cone type. As the main focus of this thesis is on dichromatic patients, we will only discuss different types of this group in detail. However, corresponding subcategories of anomalous trichromacy share similar traits with the difference that instead of lacking the ability to perceive a range of hues altogether, they have poor hue discrimination in that range which may cause confusion. The most common types of dichromacy are protanopia and deuteranopia, whose symptoms are so similar that they are both often referred to as red-green dichromats [5].

\subsubsection{Protanopia}

It is the case of complete absence of the "red" photoreceptors in the eye. Because of lacking the long-wavelength sensitive portion of retinal cones, people with such condition are unable to distinguish between colors in green-yellow-red section of spectrum. For people with such conditions, colors such as violet, lavender and purple all appear as various shades of blue because their reddish components are so dimmed as to be invisible [10]. Figure 1.2 displays another simulation of the same red apple as a person with protanopia would see it. As observed, the red portion of color in this apple is completely vanished leaving a dark green/gray color behind.
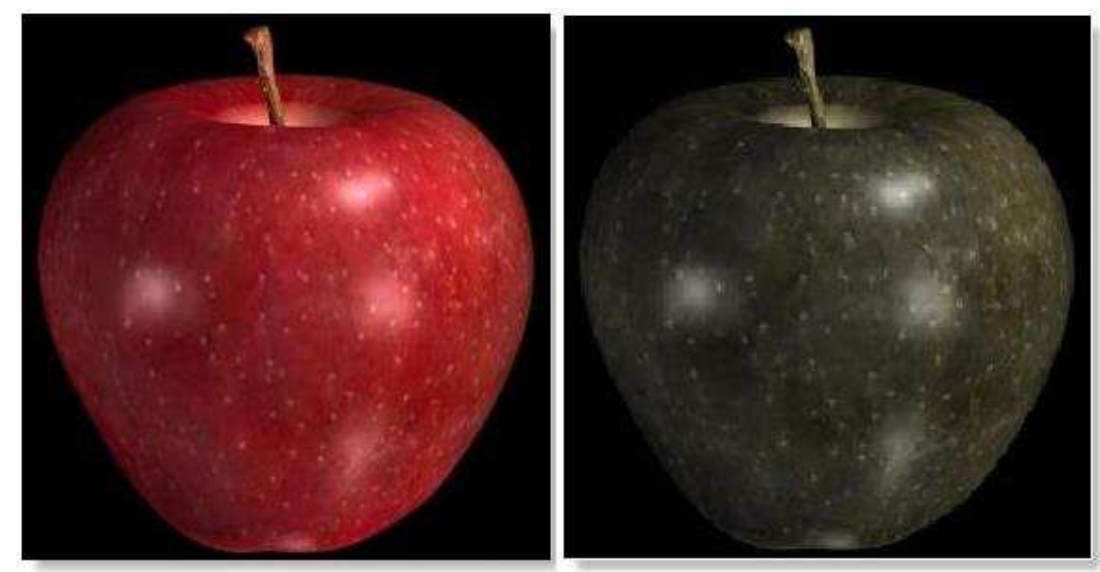

Figure 1.2: (left) The original picture (right) The simulated Protanope vision 


\subsubsection{Deuteranopia}

These types of dichromats lack the "green" retinal photoreceptors which moderately affect their discrimination of red-green hue, but without the abnormal dimming happening in protanopia [10]. Deuteranopia reduces discrimination between red, orange and yellow compared to normal. As we can see in Figure 1.3, deuteranopia has almost the same effect on the red apple as protanopia. The only noticeable difference is the lack of extreme dimming that happened in case of protanopia. Protanopia, Deuteranopia, Protanomaly and Deuteranomaly together form the group of Red-Green color deficiencies.
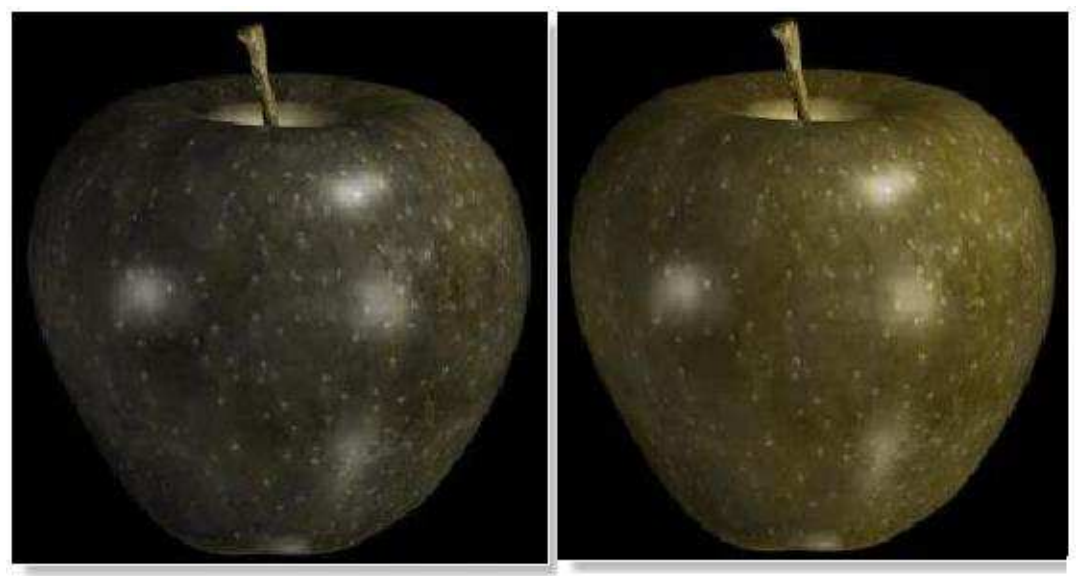

Figure 1.3: (left) The simulated protanope vision of a red apple shown in Figures 1.1 and 1.2 (right) The simulated deuteranope vision

It is worth mentioning that in case of red-green color blindness, not only red and green colors are affected but the whole color spectrum is perceived differently [1]. Figure 1.4 shows a group of colors that might cause confusion for a person with red-green color vision deficiency. As you can see it is not only the primary colors ${ }^{1}$ that might be a problem for such patients.
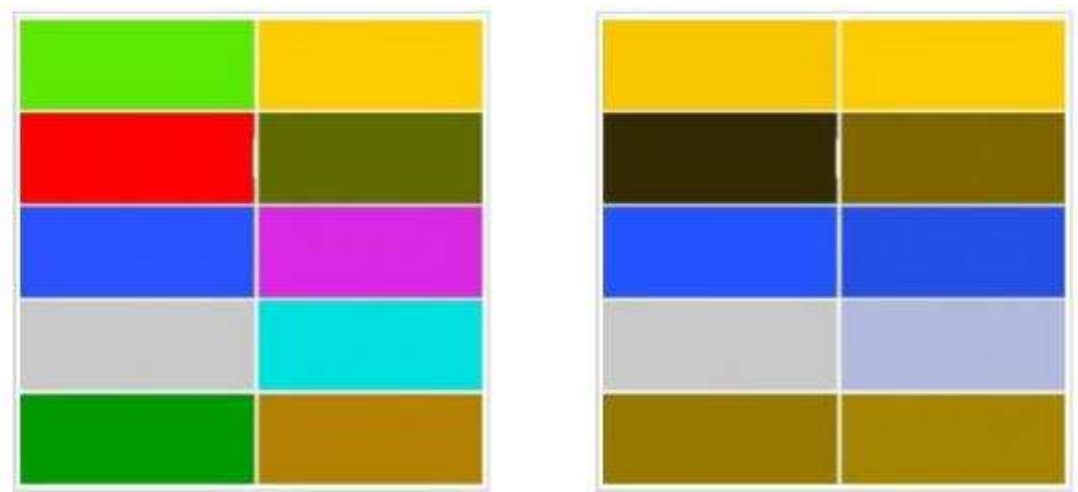

Figure 1.4: (left) The original picture (right) The way a red-green color blind perceives it

\footnotetext{
${ }^{1}$ Primary colors consist of red, green and blue. More details will be provided in Section 2.1.
} 


\subsubsection{Tritanopia}

The last sub-category of dichromacy is called tritanopia. This group is referred to as BlueYellow color deficiency. As figure 1.5 demonstrates, a general confusion between blue and green colors is expected for a person with tritanopia ${ }^{1}$. Tritanopia is non-sex-related and very rare [2].
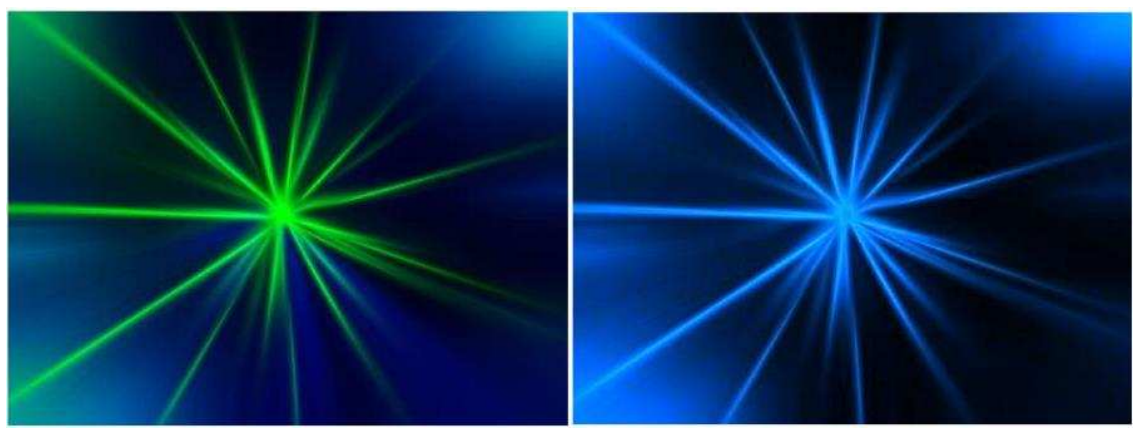

Figure 1.5: (left) The original picture (right) The simulated tritanope vision

For a tritanope, yellow color will either disappear or appear as light shades of red as shown in Figure 1.6. Patients suffering from this type of CVD see the red apple in Figures 1.1 and 1.2 exactly as a person with normal color vision would see ${ }^{2}$.
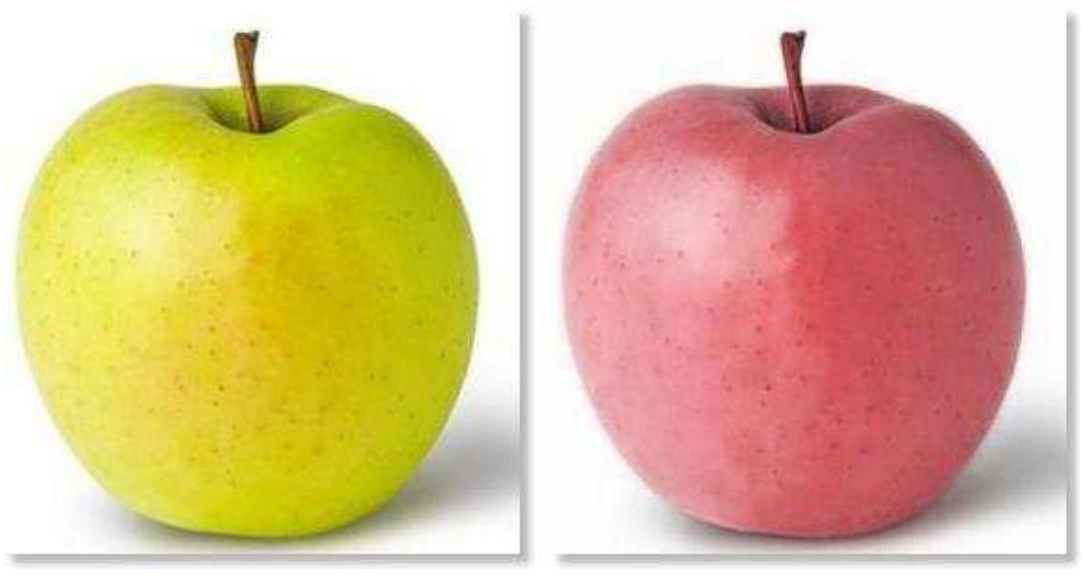

Figure 1.6: (left) The original picture (right) The simulated tritanope vision

\footnotetext{
${ }^{1}$ There are different simulation tools that try to give users with normal color vision a taste of how the world can be seen through the eyes of a person with CVD. This is especially interesting for webpage developers to know what colors might be confusing in case a color blind user tries to surf their website. The basics of how these simulation tools work, is presented in Chapter 2. However, the simulations presented so far and throughout the rest of this thesis are mainly done using the existing online simulators etre (http://www.etre.com/tools/colourblindsimulator/), coblis (http://www.colblindor.com/coblis-color-blindness-simulator/) and ASP.NET RESOURCES Color Blind Simulator (http://aspnetresources.com/tools/colorBlindness) .

${ }^{2}$ The discussions presented in this section were collectively gathered from reference presented, but the Wikipedia page on "color blind" was also used to create a comprehensive understanding of the basics of CVD which is necessary for our main goal in this thesis. http://en.wikipedia.org/wiki/Color_blind
} 


\subsection{Diagnosis}

Typically, the public usually don't see color vision deficiency as a serious problem. That is because most people do not understand the concept entirely. Moreover, most people usually believe that CVD is a scarce condition and not many people are engaged with it. That, however, is completely untrue. If you consider the statistic given in the last section, The United States alone could have over 25 million males with color vision deficiencies. You can also expect one out of every 12 man to have some sort of color vision deficiency

From the inability to perceive the beautiful world around you the way it is to not being able to distinguish the red traffic light, these are all small setbacks a color vision deficiency can bring into your life. Richmond Products in [4] lists 100 different occupations where employees may require a verified normal color vision. These jobs ranging from "Air traffic controller" to "Weather analysts" all have one thing in common: they are all in utmost need of correct color perception. That is why many of these jobs require the applicants to somehow verify their intact color vision. If color vision proves to be defective, further testing might be required to determine the type of color vision deficiency and how severe the case is.

There are various ways to test one's color vision. The "Anomaloscope" is one instrument scarcely used to diagnose color vision defects. The reason it is not pervasive is the fact that it is expensive, not commonly available for clinical use, and complex to use compared to other types of tests. "Pseudoisochromatic Plates" are the most popular method, mainly because they are very easy to use and have proven to be highly successful. The principle is based on patient's ability to recognize figures (mostly numbers, letters or shapes) within colored dots of varying sizes. While spotting these hidden figures in the middle of the colored dots proves to be intuitively easy for a normal color vision, those with CVD cannot recognize some or all shapes, based on their type or severity of CVD. Figure 1.7 displays an example of frequently used Ishihara pseudoisochromatic plate test [4].

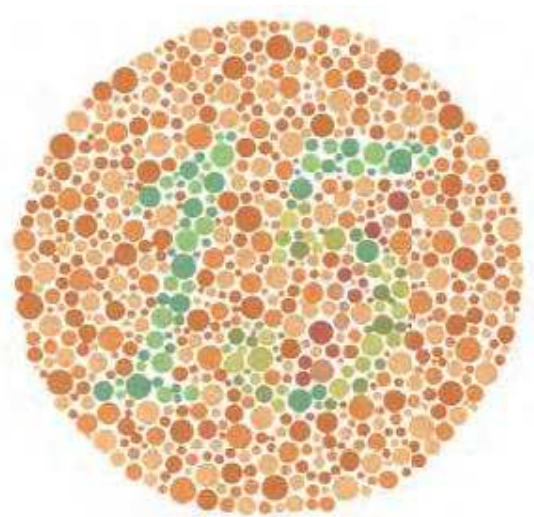

Figure 1.7: A sample of Ishihara test. The number 15, which is easily perceivable for a person with normal color vision, is nearly impossible to spot for a red-green color blind 
Although most people relate the term color blindness test directly to the dotted pictures similar to Figure 1.7 or even to the name Ishihara, this is not the only one, not the best one and certainly not the most current test available. Nevertheless, it is still frequently used all around the world and seems to continue giving satisfactory results $[1]^{1}$.

\subsection{Research objective}

A person with severe color vision deficiency might have problem in many daily routines that we intuitively do every day. For example, they might not be able to tell the difference between cooked and raw meat by its color. Sometimes it is difficult for them to distinguish between raw and ripe fruits, say bananas [2]. It might be hard for them to spot their destination on a metro map or any colored map for that matter. These and many more could be the drawbacks of being born with such condition. Specially, in the recent decades using computers and mobile phones seem to be inevitable for everyone, which brings a whole new generation of problems for such patients.

It seems that a research team led by Dr. Jay Neitz may have found a cure for congenital CVD through their research on gene therapy. The procedure has been tested on monkeys and is undergoing efficiency and safety tests [1, 4]. Although that would solve the problem for generations to come - should it reach the practical states - for the time being improving the quality of life for those suffering from this condition seems to be an interesting field of research. The approach in few other ongoing research projects is to somehow shift the color hue from the confusing range for each type of CVD into another, less confusing hue range. This is also the case for us, but we only focus on the practical side of the problem rather than aesthetics.

The main focus for this thesis is to maximize the information perception of a red-green color blind from a picture shown on a display, e.g. a monitor. To delve more into our aim, we do not care if the resulting picture does not look pretty or even has weird unrealistic colors for some objects in it. Our goal is to make a practical tool for the people with this kind of color deficiency so that they can make sure if for instance, they face a colored authentication picture online they won't have to ask someone to read it for them. That is just an example; in general we want to verify the effectiveness of our method by checking if they can pass different color vision tests after we apply our recoloring algorithm on them.

As for the reason why we are concentrating on red-green type, it suffices to say that it is by far the most common type of inherited CVD so finding an answer for it means finding a solution for a large percentage of color blinds. Moreover, if we find a technique for one type, it will be quite easy to apply the same technique to the other types just by doing a small configuration.

\footnotetext{
${ }^{1}$ There are other types of color vision deficiency tests which are outside the scope of this thesis. Further information on these tests is available in "Color Blind Essentials [1]" which can be downloaded from http://www.colblindor.com/color-blind-essentials/
} 


\section{Chapter 2}

\section{Digital Color Imaging \& Simulation tools}

The color that humans and some other animals perceive from an object is determined by the nature of the light ${ }^{1}$ reflected from that object. An object that favors reflectance in a certain range of the visible spectrum is perceived as some shades of that color ${ }^{2}$. For instance, green objects reflect light with wavelengths primarily in the range of 500 to $570 \mathrm{~nm}$, while they absorb most of the energy at other wavelengths [3]. In air or a vacuum, the visible range of the spectrum is typically specified by the wavelengths in the range of 360 to $830 \mathrm{~nm}$ [11].

If the light is "Achromatic" the only attribute related to it would be its intensity. This is exactly what viewers used to see on a black-and-white television set and what a severe case of monochromatic color vision deficient would experience. But for a chromatic light there are other attributes that play a critical role on describing the quality of a light source: Radiance is the total amount of energy that flows from the light source. Luminance gives a measure of the energy an observer perceives from a light source and finally Brightness which is a subjective descriptor and cannot be entirely measured. For instance, light emitted from a source operating in the infrared region of the spectrum can have a significant radiance but since it is not visible to the observer its luminance would be close to zero [3].

During the rest of this thesis we will extensively utilize different color models to reach the best possible result for preserving maximum information inside a picture as seen by a color vision deficient user. Therefore, it is only suitable to delve into some color theory and explain how these models work in order to have a better understanding of the actual method presented in the next chapter.

\footnotetext{
${ }^{1}$ The physical stimulus for color is electromagnetic radiation in the visible region of the spectrum, which is generally referred to as "light" [11]

${ }^{2}$ There is no color without an observer; hence attributing a color to an object is not entirely accurate. This attribution is referred to as "the stimulus error" [11]

${ }^{3}$ Void of color.
} 


\subsection{Primary and secondary colors}

As mentioned in the last chapter, cones are the sensors in the eye responsible for the color vision. We also explained how these cones are divided into three groups each sensitive to different ranges of wavelengths which are perceived as red, green or blue. Approximately $65 \%$ of all cones are sensitive to red light, $33 \%$ are sensitive to green light, and only about $2 \%$ are sensitive to blue. However, it is important to know that despite lower quantity of cones sensitive to blue, they are most sensitive which makes up for the quantity shortage [3].

The reason these three colors are always mentioned and have such significance is the fact that they are colorimetrically independent meaning they are from a collection of spectra such that the color of any one cannot be visually matched by any linear combination of the others [11]. That is why red, green and blue (RGB) are usually referred to as Primary Colors. This however does not mean that every possible color in the world can be composed directly from a linear combination of these three and only these three. For the purpose of standardization, the $\mathrm{CIE}^{1}$ designated three specific wavelength values to them: Blue $=435.8 \mathrm{~nm}$, Green $=546.1 \mathrm{~nm}$ and Red $=700 \mathrm{~nm}^{2}[3]$.

Figure 2.1 demonstrates how primary colors can mix and compose secondary colors. A secondary color is defined as a color that subtracts or absorbs a primary color and reflects or transmits the other two [3]. For example, an object perceived as yellow subtracts all the wavelengths in the blue region of spectrum and passes red and green.

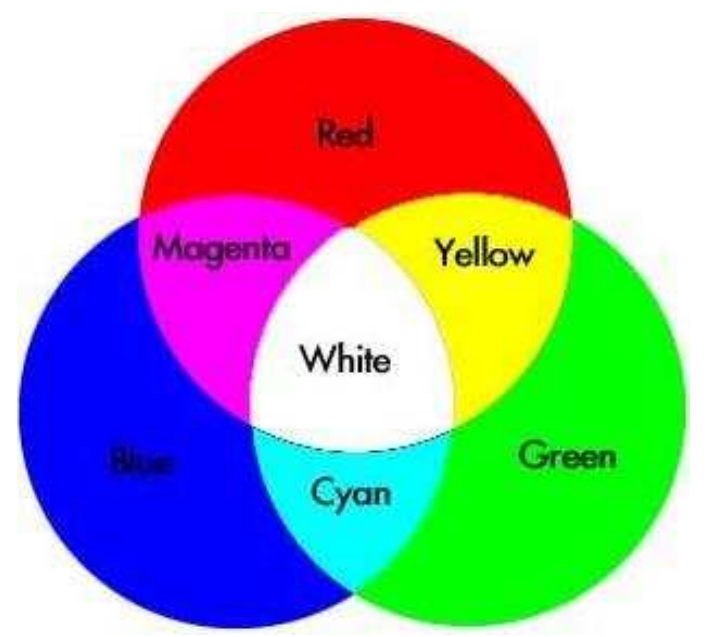

Figure 2.1: Primary and secondary colors of light

\footnotetext{
${ }^{1}$ Commission Internationale de l'Eclairage (the International Commission on Illumination)

${ }^{2}$ Although these values are set for red, green and blue for the sake of standardization, no single color can be called red, green or blue. Instead a range of wavelengths would appear red, green or blue to the observer.
} 


\subsection{Chromaticity diagram}

The characteristics usually used to distinguish one color from another are hue, saturation and brightness. Brightness is the achromatic notion of intensity. The color portion of a light source is called Chromaticity which is made of hue and saturation.

Hue is an attribute associated with the dominant wavelength in a mixture of light waves. In other words, the dominant color perceived by an observer is the hue. For example, when we call an object orange, we are referring to its hue. Saturation is concerned with the amount of white light mixed with a certain hue. As a matter of fact, saturation refers to the relative purity of a color. The pure spectrum colors are fully saturated. Colors like pink (red+white) and lavender (violet+white) are less saturated. The more white light added to them, the less saturated they become [3].

The amounts of red, green and blue needed to form any particular color are called the tristimulus values ${ }^{1}$. These values denoted as $\mathrm{X}, \mathrm{Y}$, and $\mathrm{Z}$ for red, green and blue are calculated as follows:

$$
\text { Eq2.1: } \quad x=\frac{\mathrm{X}}{\mathrm{X}+\mathrm{Y}+\mathrm{Z}}(\text { red }), \quad y=\frac{\mathrm{Y}}{\mathrm{X}+\mathrm{Y}+\mathrm{Z}} \text { (green) } \quad, \quad Z=\frac{\mathrm{Z}}{\mathrm{X}+\mathrm{Y}+\mathrm{Z}} \text { (blue) }
$$

Which leads to:

Eq2.2:

$$
x+y+z=1
$$

One approach for specifying colors is to use CIE chromaticity diagram (Figure 2.2) which shows color composition as a function of $\mathrm{x}$ and $\mathrm{y}$. By putting the tristimulus values in Eq2.1 we will reach the composition of the desired color. For example, for the specific color marked as "GREEN" in the Figure 2.2, by putting the corresponding values of $\mathrm{x}$ and $\mathrm{y}$ into Eq2.2 and then Eq2.1 and solving it, we reach the composition. It is composed of $62 \%$ green, $25 \%$ red and $13 \%$ blue.

Pure spectrum colors are indicated at the boundary of this diagram; from violet at 380nm to red at $780 \mathrm{~nm}$. All the other points inside the diagram but not necessarily on the boundary represent some mixture of spectrum colors. An interesting fact about this diagram is the fact that it is very useful for color mixing. A straight line between two points in this diagram defines all the different color variations that can be obtained by combining those two colors additively [3].

\footnotetext{
${ }^{1}$ These tristimulus values obtained from CIE XYZ color matching functions are called CIE XYZ tristimulus values. There is also tristimulus values obtained from CIE RGB CMFs but they are rarely used in most color imaging applications [11].
} 


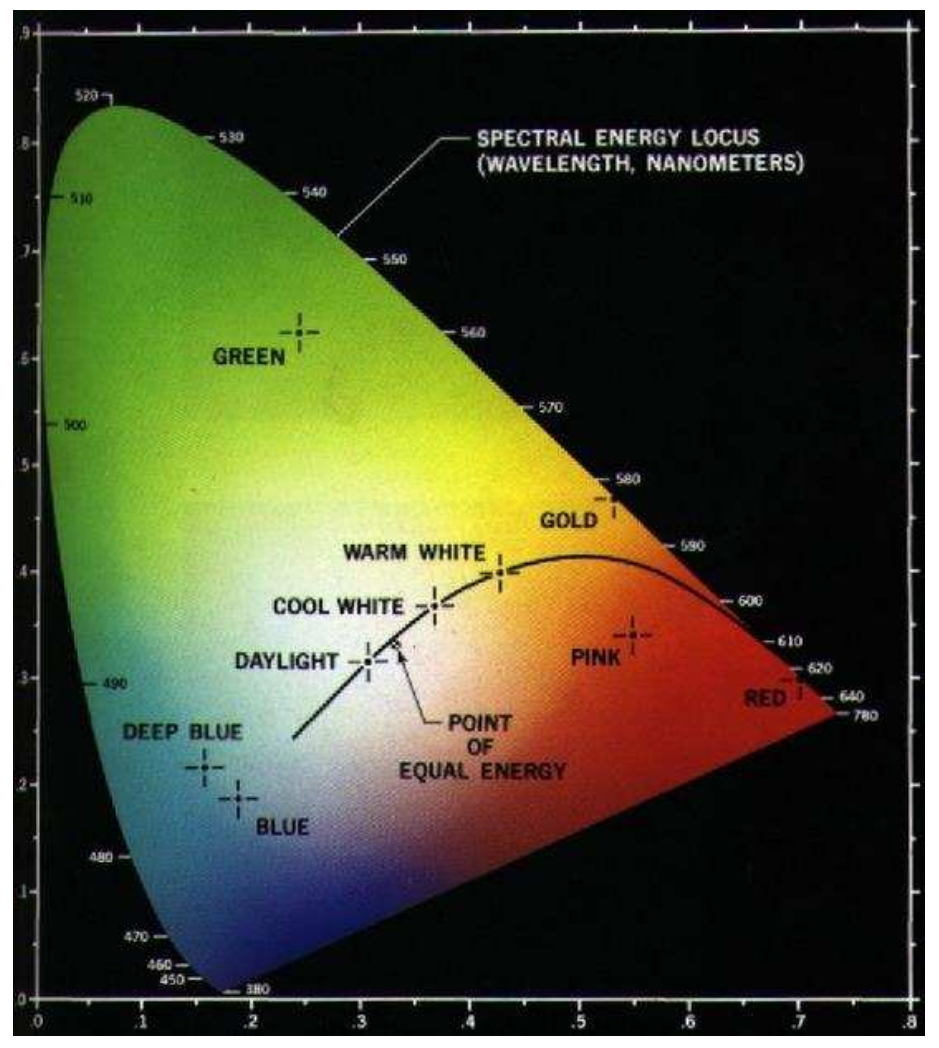

Figure 2.2: Chromaticity diagram [3]

\subsection{Color models}

A color model is a specification of a coordinate system and a subspace within that system where each color is represented by a single point. Among the various color models we decided to concentrate on RGB, HSI ${ }^{1}$, CMY/CMYK and LMS. The RGB model is widely used for color monitors and a broad class of color video cameras. The CMY model works better for output devices like color printers. The HSI model, which in fact is very similar to the way humans describe and interpret colors, has the advantage that it decouples the color (chromaticity) and gray-scale (intensity) information in an image. Therefore, many traditional image processing techniques are applicable to this model with some modifications [3].

In this section we briefly introduce these color models ${ }^{2}$ as we will need them in our method in order to change the color in an image in a way that improves the perception of a color vision

\footnotetext{
${ }^{1}$ In some literatures this color model is referred to as HSV (hue, saturation and value). These two abbreviations can be used interchangeably.

${ }^{2}$ We will explain the LMS color model during the discussion about the simulation tools later on in this chapter.
} 
deficient user from that image. However, more details on how we convert these models from one to another will be presented in Chapter 3 while we explain our method.

\subsubsection{The RGB color model}

This color model is based on the Cartesian coordinate system. As we can see in Figure 2.3, the primary RGB values are at three corners while black is at the origin and white is at the corner farthest from the origin. If all three values are equal, the resulting color will be a shade of gray lying on the Grayscale line in the image below [12]. Note that in this picture the color values have been normalized.

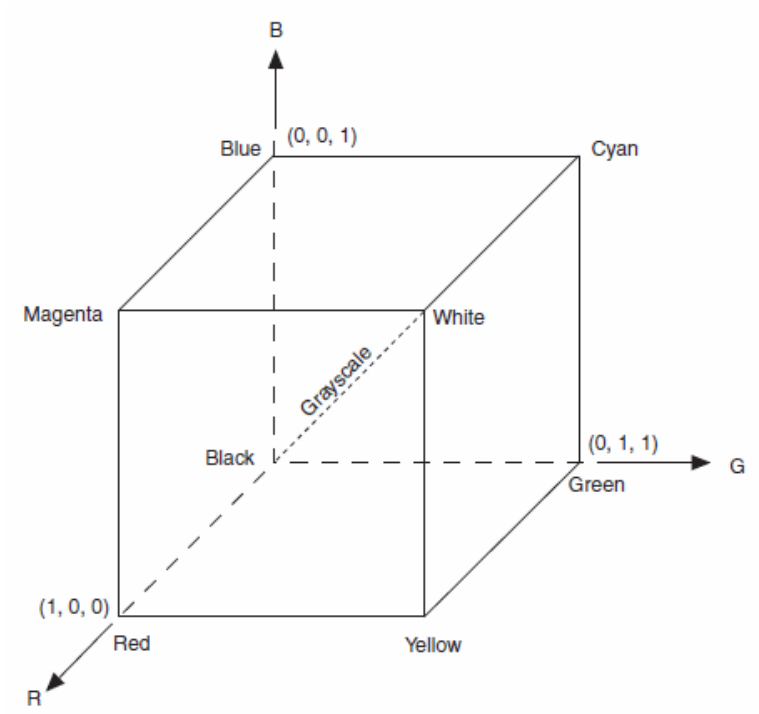

Figure 2.3: Schematic of the RGB cube [3]

Images represented in this color model consist of three component images or in other words three different image planes, one for each primary color. When we display them on an RGB monitor, these three images combine on the screen to produce a composite color image.

The number of bits used to represent each pixel in this model is called pixel depth. For an RGB image in which each of the red, green and blue images is an 8-bit image, RGB color pixel will have a pixel depth of 24 bits ( 3 image planes times the number of bits per plane). Such an image is also referred to as 24-bit RGB color image. In a typical 24-bit RGB image ${ }^{1}$, there can be $\left(2^{8}\right)^{3}$ different colors, though the number of colors that can actually be represented on a device, say monitor, is limited by its gamut ${ }^{2}$.

\footnotetext{
${ }^{1}$ Such 24-bit RGB color image is usually referred to as "full-color image"

2 A color Gamut consists of all the colors which are realizable with a particular color model or medium.
} 
To generate color planes, we can keep one of the three primary colors constant and allow the other two to vary. For example, we can have a plane parallel to GB-plane passing through the center of the cube by having a fixed coordinate of 127 for red and changing $\mathrm{G}$ and $\mathrm{B}$ components as $(127, \mathrm{G}, \mathrm{B})[3]$.

\subsubsection{The CMY and CMYK models}

Color output devices can be classified into two main types: additive and subtractive. Additive color systems combine different primary colors on a dark background to produce the desired color. The idea is that the final spectrum is the sum (or average) of the spectra of the individual lights coming together. That is exactly how the RGB model works. On the other hand color in a subtractive system is produced through the process of removing (subtracting) unwanted spectral components from "white" light.

Typically subtractive systems are based on cyan, magenta and yellow (CMY) colorants that absorb light in the red, green and blue respectively ${ }^{1}$. Each colorant absorbs its complementary color and transmits the rest of the visible range of the spectrum [11].

As mentioned earlier this color model is used mostly for color printing. For instance, when a surface coated with cyan pigment is illuminated with white light, what happens is that cyan subtracts red light from the reflected white light. In a perfect world converting RGB to CMY values should be a very easy task, simply subtracting red from 1 to reach $\mathrm{C}$ value, green from 1 to reach $\mathrm{M}$ value and blue from 1 to reach $\mathrm{Y}$ value [3]. However, in reality the printed colors of CMY are far from ideal and this conversion can actually be device dependant.

It is also common to use a fourth colorant (black color abbreviated $\mathrm{K}$ ) that absorbs light almost uniformly over the visible spectrum. This improves the gamut by allowing the production of darker colors and also better reproduction of achromatic (gray-scale) colors. This addition also reduces the costs through the replacement of the more expensive CMY colorants with the black. This new color model is referred to as CMYK [11].

\subsubsection{The HSI color model}

The RGB color model matches nicely with the fact that human eye is very good at perceiving red, green and blue primaries. However, models like RGB or CMY are not well suited for describing colors in the ways that humans usually interpret them. For example, when we see a car in the street we do not say that car has $60 \%$ blue, $30 \%$ red and $10 \%$ green! We simply call that car violet which in fact is the hue of that car.

\footnotetext{
${ }^{1}$ The red, green and blue spectral regions are roughly defined as the intervals $600-700,500-600$ and $400-500 \mathrm{~nm}$, respectively [11].
} 
The HSI color model (hue, saturation and intensity), as mentioned before, decouples the intensity component from the color-carrying component (hue and saturation) in a color image. We will discuss the conversion between RGB and HSI color models in detail in the next chapter but just as an introduction, we can determine the hue of a color by calculation the angle from that color to the red axis $\left(0^{\circ}\right.$ angle) in Figure 2.4. The length of the vector from the center of this circle to the point of our desired color gives us the saturation of that color [3]. A saturation value of zero (in the center of the circle) means that the color is achromatic (gray). If the specific color we are interested in lies on the outer boundary of this color wheel, it will have the maximum saturation for that specific hue and intensity. Finally, intensity is the overall lightness or brightness of the color.

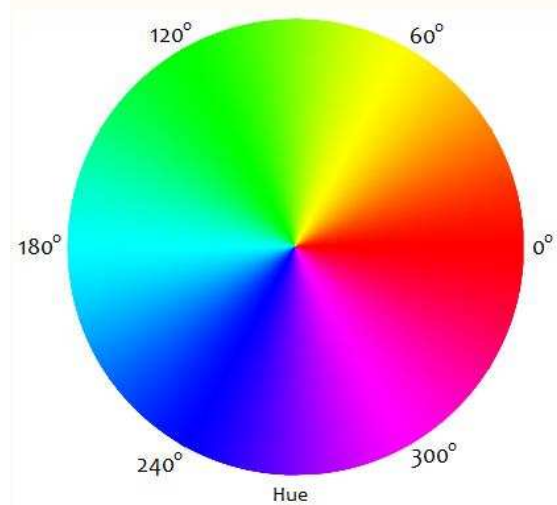

Figure 2.4: A color wheel for determining the hue and saturation of a specific color

Figure 2.5 demonstrates the overall view of the HSI color model. In this model, for each intensity level, we will have a color plane with different hues and saturations.

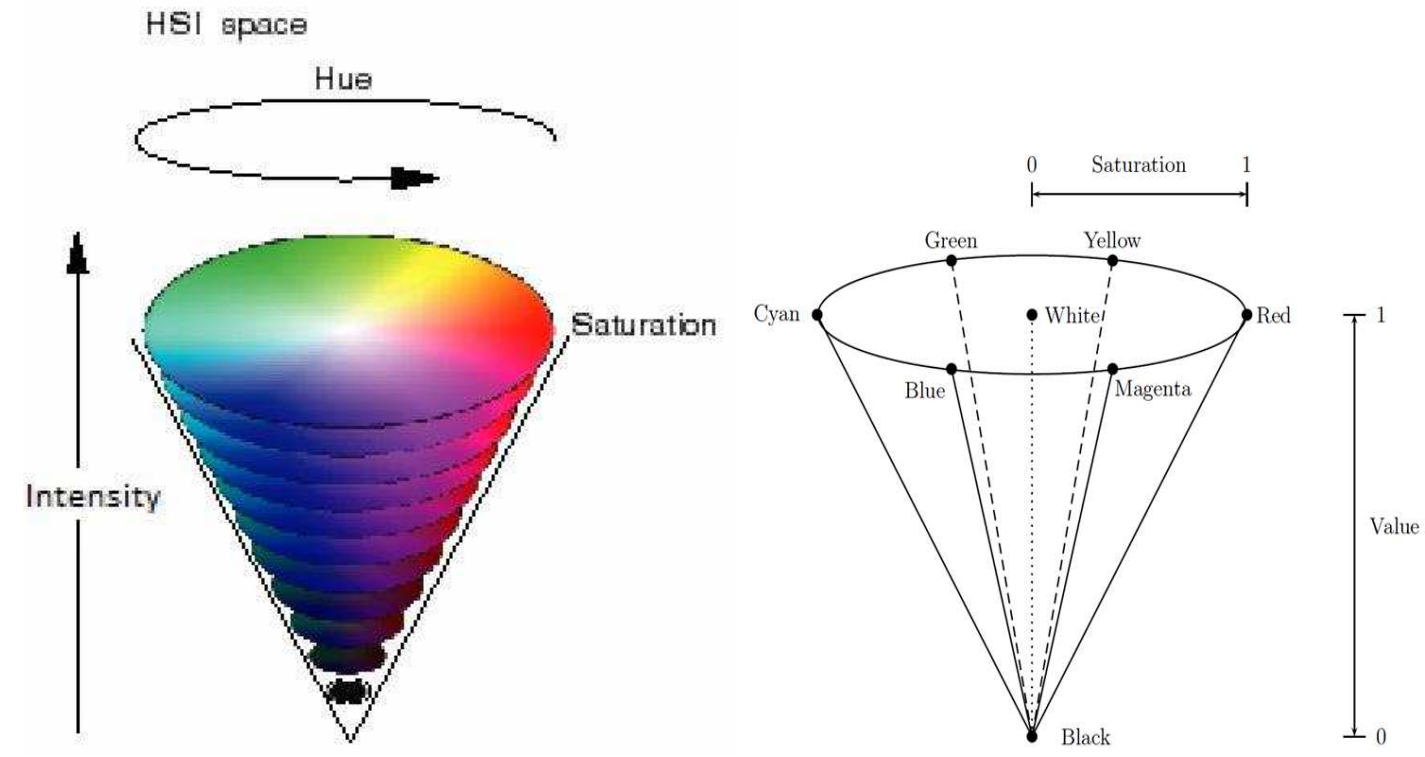

Figure 2.5: The HSI ( HSV) color model 


\subsection{Full-color image processing}

In this thesis we will be challenged with a range of image processing tasks in order to improve the perception of a color vision deficient from a full-color image. It only seems suitable to discuss the basics of how typical image processing techniques can be applied to a 24-bit color image. Full-color image processing approaches fall into two major categories. In the first one, each component image is processed individually and then these processed image components come together to form a composite final image. In the other approach, we work with color pixels directly. Color pixels can be treated as vectors since they have at least three components. For instance, in case of a pixel in RGB color system we can show it as:

Eq2.3:

$$
\mathrm{c}(\mathrm{x}, \mathrm{y})=\left[\begin{array}{l}
R(x, y) \\
G(x, y) \\
B(x, y)
\end{array}\right]
$$

For an image of size $\mathrm{M}^{*} \mathrm{~N}$, there are $\mathrm{MN}$ such vectors, where (x,y) is the pixel coordinates. A great advantage of having color pixels is the fact that it allows us to process a color image by processing each of its component images separately, using standard gray-scale image processing methods without even being concerned about the color. However, the results of these two approaches are not necessarily always the same [3].

\subsection{Simulation tools}

As we mentioned before, daily lives of a large number of people is affected by color vision deficiencies but at the same time it is difficult for others to understand the experience of having CVD. One technique that can help to improve this understanding is using simulation tools. Moreover, we needed to somehow verify if the changes that our color transformation method brings to the images, is going in the right direction. That is why we needed to simulate the vision of a color vision deficient user.

We could either implement our own simulation tool or use the existing tools. Since the main focus of this thesis is to improve the perception and not study the perception itself, we decided to use an existing online color blind simulation. The only important fact to note is that the simulation tool needs to be unanimous. If we test one picture with one simulation tool, in order to reach a valid conclusion, same simulation tool needs to be applied to all other test images as 
well. That is exactly what we did throughout this thesis. However, we felt like it is beneficial to know the basics of such simulation tools.

There are various approaches to simulate defective vision for normal observers, as mentioned in [13-17]. The earliest approach amongst them was introduced by Meyer and Greenburg in 1988 [17]. This approach translates colors within a color space in order to model dichromatic color vision. Color science researchers extended this approach to provide a better algorithm for simulating dichromacy $[13,14,18]$. These simulations show how images would look like for people with any of the three types of dichromacy.

In some other efforts researchers worked on simulation of anomalous trichromacy [19, 20]. These types of simulations require both the type of anomalous trichromacy and additional information about the severity of the condition. This additional information is typically expressed in the amount of peak sensitivity shift of the anomalous cone. The problem is that this type of information is not readily available from the existing color-vision tests [2].

\subsubsection{Dichromatic CVD simulation}

The process of simulating the appearance of a digital image as it would look like for a dichromatic user consists of replacing the color of each pixel in the image by the color perceived by the person with CVD. This is a compressive process as people with CVD perceive fewer colors than people with normal color vision, i.e. different original colors will map to the same replacement colors. For example, people with protanopia perceive particular shades of gray, pink and turquoise all as a single gray [2]. In this process, both the set of colors that are perceived identically and the color that they should be mapped to need to be identified.

A common approach for simulation of a color-deficient viewer is the procedure introduced by Meyer and Greenberg [17]. They engineered their approach based on empirical studies on people with unilateral dichromacy ${ }^{1}$ which suggest that the $2 \mathrm{D}$ hue values of normal vision are reduced to a single dimension that is linear in terms of the CIE Uniform Chromaticity Scale coordinates:

$$
\text { Eq 2.4: } \quad u=\frac{4 X}{X+15 Y+3 Z} v=\frac{9 Y}{X+15 Y+3 Z}
$$

For each type of deficiency, a pair of line segments in uv space is identified, where each pair connects a reference white value to two specific spectral energies, as shown in Table 2.1. To determine where, on such line segments, a color-deficient viewer will perceive any specific

\footnotetext{
${ }^{1}$ It's a condition in which the patient has normal view in one eye and dichromatic vision in the other. More details will be available in Chapter 3 .
} 
color, another color space called LMS (sometimes referred to as SML) is used. This color space is named after the three components corresponding to cone sensitivities in the visible spectrum's long, medium, and short wavelength regions.

\begin{tabular}{lcc}
\multicolumn{2}{c}{ Spectral energies } \\
\hline Deficiency & $\begin{array}{c}\text { Energy 1 } \\
\text { (nanometers) }\end{array}$ & $\begin{array}{c}\text { Energy 2 } \\
\text { (nanometers) }\end{array}$ \\
\hline Protanopic & 473 & 574 \\
\hline Deuteranopic & 477 & 578 \\
\hline Tritanopic & 490 & 610
\end{tabular}

Table 2.1: Spectral energies

Meyer and Greenberg [17] provide a linear transformation between CIE XYZ ${ }^{1}$ space and LMS space, given by Eq 2.5:

$$
\text { Eq 2.5: } \quad\left[\begin{array}{c}
S \\
M \\
L
\end{array}\right]=\left[\begin{array}{ccc}
0.0000 & 0.0000 & 0.5609 \\
-0.4227 & 1.1723 & 0.0911 \\
0.1150 & 0.9364 & -0.0203
\end{array}\right]\left[\begin{array}{l}
X \\
Y \\
Z
\end{array}\right]
$$

At this point any color deficiency can be expressed as an inability to recognize the variations in one of these components. For instance, a user with protanopic deficiency would be unable to distinguish the differences in the L component. Therefore, the confusion line in LMS color space for such a user would be a line with fixed values of $S$ and $M$ and varying values of $L$ component.

In order to recognize the perceived color corresponding to any original color in RGB color space, RGB color needs to be converted to CIE XYZ space and then mapped to LMS space as explained before. Based on this transformation, for each deficiency a specific confusion line in LMS space can be determined. By applying the inverse of transformation presented in Eq. 2.5, this confusion line can be projected onto uv space (see Eq. 2.4). Next step is to find the intersection of that line with one of the line segments previously mentioned, based on the specific deficiency at hand. The resulting uv coordinates of this intersection, together with the luminance of the original color, generate the perceived color.

\footnotetext{
${ }^{1}$ This color space was originally defined to avoid the negative values that sometimes occurred in CIE RGB color matching functions. Basically CIE XYZ is a linear transformation of CIE RGB [11].
} 
To summarize, we can say that most simulation approaches represent color stimuli as vectors in a three dimensional LMS space and then simulate the dichromatic vision by collapsing one of these three dimensions into a constant value. The LMS values of any given color stimulus can be discovered from the measured spectral power distribution corresponding to the stimulus. The simulation for dichromatic vision can be approximately regarded as projecting the threedimensional color space onto a two-dimensional plane, or two intersecting planes with similar normal orientations. Figure 2.6 depicts this dimension reduction for a dichromatic user.

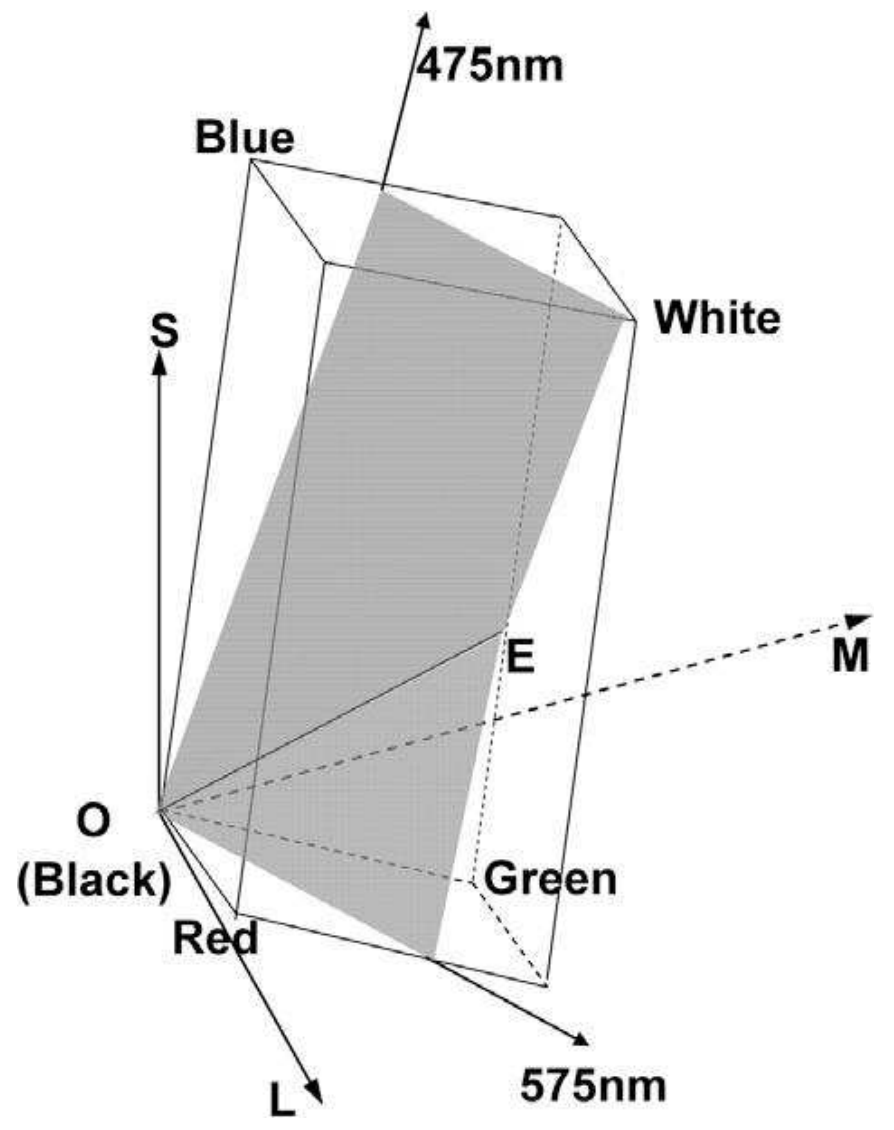

Figure 2.6: A simple sketch of the color dimension reduction representing the reduced color space of dichromatic users [13] 


\title{
Chapter 3
}

\section{Proposed Color Transformation Method}

\begin{abstract}
Although in the near future researchers might finally introduce a cure for color vision deficiencies, for the time being individuals with such problems need to somehow deal with these problems in their day to day life. Because of this, a growing number of researchers are striving to improve the quality of life for CVD patients. In the next section, we will present a general overview of such attempts. However, our main focus in this thesis will be on the color transformation methods.
\end{abstract}

\subsection{Background}

Since color vision deficiency was discovered by John Dalton ${ }^{1}$ in the $18^{\text {th }}$ century, different rectification methods have been proposed. Adjusting or redesigning the colors of the real world around us could be a way to help color vision deficient individuals avoid parts of their color confusion, but this method cannot be applied everywhere [5]. Moreover, changing everything to help roughly 8-10 percent of the population would seem inconvenient for the remaining part of the society who have normal color vision and are used to certain types of colors in their daily lives. Therefore, there have been ongoing researches in different fields to help color blind people while respecting people with normal color vision. We can categorize these activities into four main groups:

Cure: First group of scientists are trying to find a cure for the whole thing. Researchers such as $\mathrm{K}$. Mancuso et al. [21] explore the possibility of curing color vision deficiencies by using gene therapy. Experiments on adult monkeys have been carried out and testing effectiveness and side

\footnotetext{
${ }^{1}$ The English chemist who published the first scientific paper on color vision deficiencies in 1798 after realizing his own color blindness. More info at http://en.wikipedia.org/wiki/John_Dalton
} 
effects of this treatment is still in progress. As this branch of researches is more related to genetics and medical science, details are out of the scope of this thesis.

Equipment: Another way to improve quality of life for people with color vision deficiency would be introducing different instruments to remove some of the color confusion they face every day. Some personalized glasses with different tinted lenses have been introduced which can help CVD people to distinguish colors. The way these glasses work is very simple; the patient uses colored lens on his non-dominant eye. This allows both of his eyes to perceive different colors. Hence, both sides of the brain have to work together in order to gather the information from the side that uses colored lens.

An alternative would be using colored lenses for both eyes, just with different colors which is a crucial part of this method. It will not work if lenses on both eyes use the same color simply because it eliminates the whole idea of stimulating both sides of the brain. This technique could potentially work with every case of color vision deficiency with various degrees of severity except people with total color blindness [22]. Although these glasses could be useful for the color blinds, they also tend to mask out some useful color information and cause other color confusions [5].

On the other hand, researchers such as C. L. Lai and S. W. Chang [23] and Kim et al. [10] propose image based portable auxiliary systems to integrate multiple perceptual aids for visually impaired people. Such systems are not necessarily limited to color vision deficiencies and can be configured for various visual impairments such as poor vision.

A clear usage of such a system could be a real time traffic light recognition system based on computer vision [10]. A typical traffic light often has the same order about the placement of red, green and yellow lights in it and a CVD individual needs to simply act based on which part of the traffic light appear brighter to him, even without the knowledge of what color it actually represents. However, there are some limitations such as the weather condition, the environment lighting and time of the day.

In bright sunlight, red lights might look dim to a color blind driver and fail to stand out against the background of sky and the rest of the environment. On the other hand at nights, green lights look white to them which could sometimes be indistinguishable from the street lights [24]. Another problem could be traffic lights such as the one shown in Figure 3.1. As we can see there is no possible way for a dichromat to distinguish the color of the arrow-shaped light, even if the green light can be guessed by its position. These limitations validate the idea of promoting systems of such kind to ensure the safety of color blind drivers as well as the people around them. 

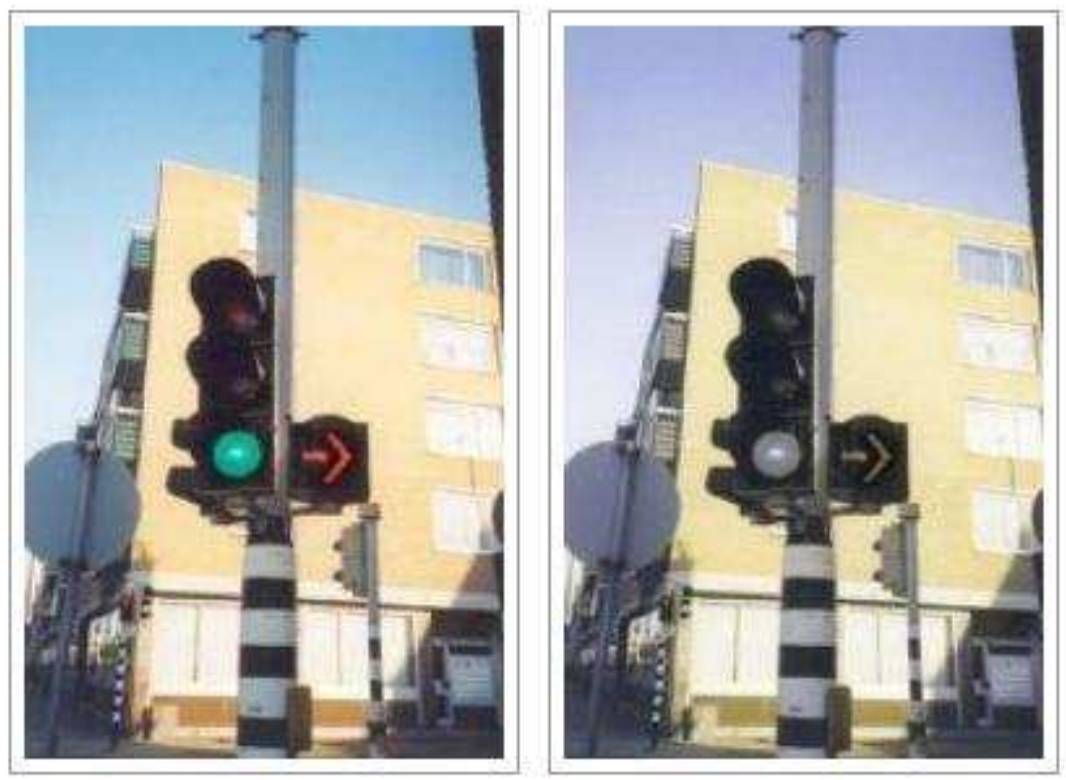

Figure 3.1: (Left) A picture of a confusing traffic light for color blinds (right) Protanope simulation of the same picture [9]

This system requires a digital video camera, a portable PC as the image processing unit that helps the system detect and track red, green and yellow traffic lights and of course a head mounted LCD to project the result on the color vision deficient eyes. By adopting such a method, personal perception details of the current user can be modeled in the beginning, then the input video stream is processed, adjusted and re-projected to the HMD to compensate the specific visual defect that user possesses [10, 23]. Kim et al. [10] also bring up the idea of using audio feeds to enhance the proposed system just as it is utilized to help the blind.

Simulation: Another interesting field in the world of color vision deficiency is simulating different types of color blindness. A lot of researches have been conducted on how to model the vision of a visually impaired individual, leading to a number of papers presenting algorithms to simulate what color blind people see [13, 14, 18, 25-27].

Methods for simulating color vision deficiencies date back to the beginning of $19^{\text {th }}$ century, when Goethe painted a water-color landscape by using colors intended to demonstrate the view as seen by a typical blue-blind observer [28]. Simulation methods are of significant importance because:

First, they are the most direct way for people with normal color vision to experience the world as a color blind sees it [5]. This is specifically important for people living with a color vision deficient. Parents of color blind children could find out how their children see the world and act accordingly. From buying toys with proper colors to choosing the cloths in a way that avoid confusion for their children, simulation tools are the only way to help such families. 
Second, having an understanding of how a particular image or webpage would look like to the population of people with color vision deficiencies helps developers choose better color setup to avoid confusion for that group of users.

Finally, simulation tools enhance the process of testing new methods in the world of color vision deficiency. Suppose a researcher trying to find a new way to improve information perception for CVD users had no idea how the resulting image would look like to an actual color blind. In such cases, every step of the way researchers had to test their results by passing it through a color blind directly. Simulation tools make it a lot easier to promote new ideas to help color vision deficient users. As we already explained the basics of common simulation tools in Section 2.5, we will not go into more details about the simulation tools here.

Color Transformation: The last group of activities in the field of color vision deficiency revolves around re-coloring and color transformation approaches. These approaches usually emphasize on the perceptual difference between various colors rather than the identification of those colors. They are often designed to adjust colors into discriminable ones for CVD users. For example in case of dichromacy, which is the topic of this thesis as well, several researchers tried to replace the confusing color contrast (e.g. the red-green contrast) into discriminable color contrast (e.g. the blue-yellow contrast) in order to improve the information perception for color vision deficient users [7, 29-31].

Although at the end of the day most of these approaches try to reach the same results, the methods on doing so differs from one to another. In the remainder of this chapter we will explain our approach in detail and present a few images as our objective result. In Chapter 4, we will introduce a few other distinct methods trying to reach similar results which will be also compared with our results.

\subsection{Method Objectives}

As we mentioned briefly in Section 1.4, our plan is to devise a method to maximize the amount of information that a color vision deficient user perceives from a picture comparing to the original, unedited image. We can reach this goal by adjusting and transforming colors in a digital image while preserving the overall contrast of the picture. We have emphasized numerous times throughout this thesis that since some colors are not entirely discriminable for cases like dichromacy, people inheriting this type of CVD might have a confusing experience finding certain details in some pictures. Therefore, if we can find a way to somehow manage to increase the amount of details they perceive from images, we can count our method as a successful approach. 
The reason we keep emphasizing on dichromacy is the fact that up until now there doesn't exist any approach to help total color blinds as they lack two or all three of photoreceptors. Moreover, although patients with anomalous trichromacy have decreased ability to perceive certain colors due to the shift in the cone sensitivity peaks, they are perfectly capable of figuring out most details in the image just like a person with normal color vision. The only difference is that they perceive slightly different colors but that does not prevent them from doing most of the daily activities everyone else does ${ }^{1}$. That leaves us with the extreme cases of dichromacy.

Dichromats fail to perceive some colors altogether and that calls for some kind of rectification. The cases of tritanopia are extremely rare so we decided to put most of our efforts on red-green colorblindness. On the other hand most protanopes and deuteranopes share similar symptoms with slightly different perceived color range [5]. Consequently, we decided to make details of our method suitable for protanopia. That being said, deducing a similar algorithm for the other cases is only a matter of some simple configurations and focusing on protanopia is solely with the aim of preventing redundancy. From now on wherever we mention dichromats we are implicitly referring to people with protanopia.

The most important point to mention is our end game. Our objective is not to transform pictures in such a way that they contain the same level of details for everyone (both groups of observers with and without CVD). Contrary to what researchers such as Huang et al. [30] try to achieve, we do not care if the resulting image seems unnatural or unpleasant for a viewer with normal color vision. For instance, we do not care if green leaves of a flower appear blue to the people without CVD in the transformed image. Our method is dedicated to help dichromats perceive some more details from certain pictures that make frustration for them.

Just like all of us, color blind users are entitled to have visual accessibility especially on media like monitors or mobile phones. We would like to think of our method as an On-Off switch for such users. Suppose a user with dichromacy is surfing through internet or shuffling through family pictures on his computer and faces a specific picture but he cannot see some part of it clearly. Our method should come in handy for those kinds of situations. He can easily transform the picture using our application and get another version of the same picture which now provides more details in terms of color discrimination. Meanwhile, if a family member with perfectly normal color vision stumbles upon that picture, it might look unnatural or weird to him/her.

Walraven and Alferdinck [32] emphasize that for many applications, the differentiation of colors is far more important than the identification of them. That is exactly what we will pursue in this thesis. We will try to keep the overall contrast of the image and mainly focus on the differences between pairs of colors rather than worrying about ending up with unnatural colors. Our objective is to first recognize the troublesome colors for our dichromatic users, then adjust those

\footnotetext{
${ }^{1}$ Anomalous trichromacy forms over $80 \%$ of the population of color blinds [28], but due to the reason described above most efforts are towards fixing more severe cases of dichromacy which have much bigger impact on patient's life.
} 
colors to different colors that are recognizable by them while maintaining the overall contrast of the picture. What we mean is that we want our CVD to perceive the same lightness in parts of the picture that are brighter and the same shadow in darker parts of the picture, only with possibly different colors.

\subsection{Method Overview}

In the previous section we explained the goals of this thesis. We will now discuss how we plan to achieve those goals. As we mentioned, we want to transform colors in the picture in such a way that while preserving the overall details and contrast of the image, the new color composition gives a confusion-free and discriminable color perception to our dichromatic patient. As we will explain in the next chapter different suggestions have been made to achieve this goal. However, the path we take is taking advantage of great facility the HSI color model presents to us.

We said that we want to keep the overall details of the picture. That is exactly what HSI color model gives us. If we can somehow convert our picture (which is typically in RGB color model originally) into HSI color model, we will have just the thing we need. Suppose this conversion has been made; in that case for every single pixel in our image, we have hue, saturation and intensity values. Therefore, by keeping the intensity constant we make sure we keep the picture exactly as it is. Now if we decide to manipulate hues of a pixel and its surroundings, we rest assured that contrast between those pairs of pixels are still preserved. Figure 3.2 shows a typical conversion of this type.
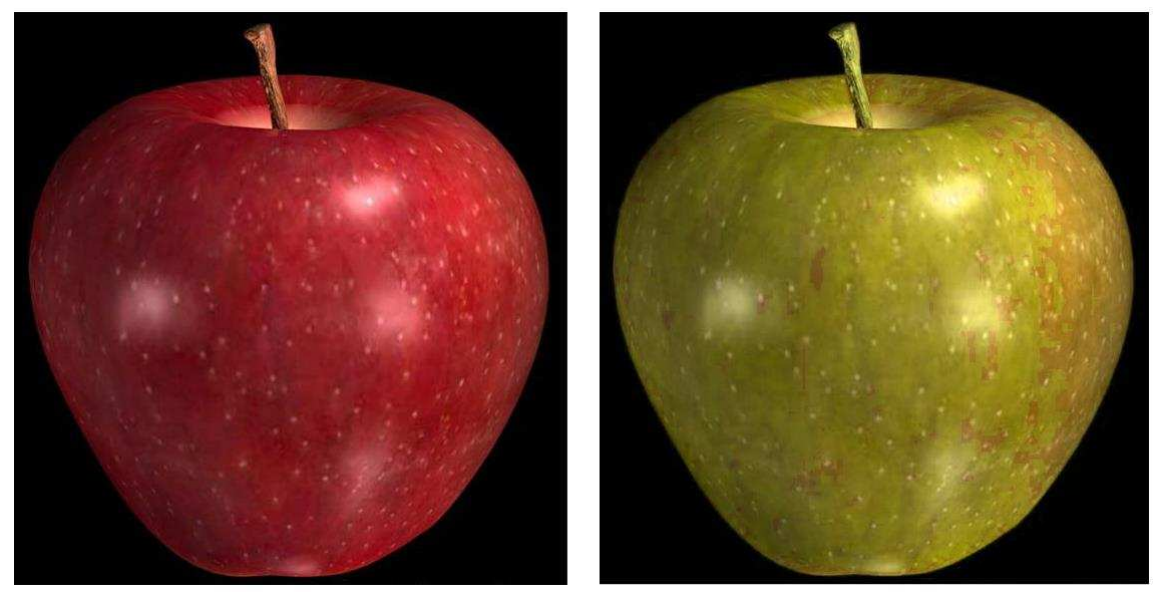

Figure 3.2: (left) Original red apple picture (right) Changing the hue from red to yellow 
It is worth mentioning that this is not the result of our actual method here. We merely picked a range of red hue and converted it into a range of yellow/green without taking a lot of details into consideration. The point here is that, as we can see, all the highlights and details in the picture are preserved but the overall color changed from red to a yellow/green shade.

It is also important to know exactly the probable color perception and color confusion of the dichromats to have a reliable result. In the next section we will discuss how we found the range of colors that might be troublesome for dichromats and the range of colors that make no confusion. Finally we need to make sure that the resulting colors don't end up generating confusion by being too close to some other unchanged colors in the original picture.

\subsection{Method Implementation}

From this point forward we start implementing our algorithm in order to reach our described goals. We picked Matlab as our implementation tool of choice, because of the powerful functions and image processing tools it offers. All the transformed images that we will present are the outputs of processing the original images through Matlab. In this section we will describe our algorithm step by step.

As we mentioned briefly in Chapter 2, a typical digital image shown on a computer monitor is presented in RGB color model. It is the same in Matlab as well. The first step is to import a test image into our code. This color image is made up of three different layers on top of each other: red, green and blue layers. Therefore, for each pixel we have 3 values, each corresponding to the value of that specific pixel in one of these layers. In other words, those values show the composition of that pixel, telling us how much red, green and blue colors have contributed to the final color of that pixel. However, RGB color model does not serve us any favor. As we mentioned in the previous chapter our ideal color model would be HSI model. Hence, in order to compensate for the missing hue range in the visual spectrum of a dichromat, we need to somehow convert our current RGB image into HSI model to be able to avoid the troublesome color range.

\subsubsection{Converting RGB into HSI}

Suppose we import an RGB image into our code. If we did not normalize pixel values while reading the image, each pixel might have values between 0 and 255, for each image plane. In that case we need to normalize all pixels so that each pixel has a value between 0 and 1 . 
We first try to find out our Hue. As we discussed briefly in Section 2.3.3, the hue is the degree between that pixel and the red axis which represents zero. The value of $\theta$ and consequently $\mathrm{H}$ are calculated from the following equations:

Eq 3.1:

$$
\begin{gathered}
\theta=\operatorname{Cos}^{-1}\left\{\frac{0.5[(R-G)+(R-B)]}{\sqrt{\left[(R-G)^{2}+(R-G)(R-B)\right]}}\right\} \\
H=\left\{\begin{array}{cc}
360-\theta, & \text { if } \mathrm{B}>\mathrm{G} \\
\theta, & \text { if } \mathrm{B} \leq \mathrm{G}
\end{array}\right.
\end{gathered}
$$

Given the normalized values of $\mathrm{R}, \mathrm{G}$ and $\mathrm{B}$, we can calculate the saturation and intensity values as follows [3]:

Eq 3.2:

Eq 3.3:

$$
\begin{gathered}
\mathrm{S}=1-\frac{3}{R+G+B}[\min (\mathrm{R}, \mathrm{G}, \mathrm{B})] \\
\mathrm{I}=1 / 3(\mathrm{R}+\mathrm{G}+\mathrm{B})
\end{gathered}
$$

So after we perform these calculations for each pixel in our image, we will end up with values in HSI color model. Again our image will have three layers: first image plane will represent hue, second one, saturation and the third image plane represents the intensity.

Although we can write a code for converting RGB pixel values into HSI, Matlab offers a function that does the same job for us. Function rgb2hsv converts every pixel from RGB space into HSV. However, since this function normalizes the RGB values itself, we need to be careful not to normalize the RGB values while reading the image file.

\subsubsection{Finding the troublesome hue range for dichromats}

At this point we have an image whose pixels are each consisting of a hue, saturation and intensity value. As we mentioned before, dichromats have problem discriminating between colors that other people easily distinguish as different. Such patients will mistake different colors for the same one. In other words, dichromats deal with reduced color gamut on a daily basis [29]. Our next step is to delve more into which colors are the origins of problem for such patients and which colors are perceived without any problem.

Special measurements from people with a condition called unilateral dichromacy gave a lot of insight about the range of colors that could make confusion for dichromats. Unilateral 
dichromacy is a condition in which the patient has dichromacy in one eye and normal color vision in the other eye. The studies show that there are specific wavelengths that are perceived identically by those with color vision deficiencies and those with ordinary vision. People with this condition identified that spectral colors of $475 \mathrm{~nm}$ (blue) and $575 \mathrm{~nm}$ (yellow) are identically perceived by people with protanopia or deuteranopia and those with normal color vision [13, 31].

Although this fact inspired us for this color transformation method, these wavelengths don't necessarily help us in any way. We have an image whose pixels are represented by hue, saturation and intensity. So what we need to do in this step is to try to find out exactly which hue range is the safe range for a dichromat and which range might make confusion.

In order to distinguish the safe range of the colors, we wrote a code to generate different hues in a square-shaped color card. We kept the saturation and intensity constant at maximum value of one throughout the whole image and just changed the hue one degree at a time. So each $40 * 40$ block of pixels in this resulting picture has a uniform hue, saturation and intensity. The next block has the same intensity and saturation but one degree higher hue. The resulting color card is shown in Figure 3.3 (top).

The next step would be simulating how this color card is seen by a protanope. We perform this simulation using an existing online simulation tool but the information about the basics of how such a simulation tool works, is explained in Section 2.5. The protanope simulation of our color card is shown in Figure 3.3 (bottom). Although we can manually distinguish where the two images have the same colors and where they are perceived differently, we decided to write another piece of code to automatically distinguish the exact hue range of the safe and troublesome colors.

This process is carried out by comparing the hue of each cell block in the original color card with that of the corresponding cell block in the simulated protanope color card. If the difference between the hue of a certain block and the hue of its corresponding block in the simulated image is less than a threshold, we count that hue as one of the safe colors for our protanope patient. That means the patient perceives colors of that hue roughly the same as everyone else.

This is one of the qualities that make our method different from similar methods presented in [6, 23 , or 33]. We can precisely pinpoint the safe range of hues for a color vision deficient user and transfer all colors that generate confusion for patient into this range without risking of entering another troublesome range. 

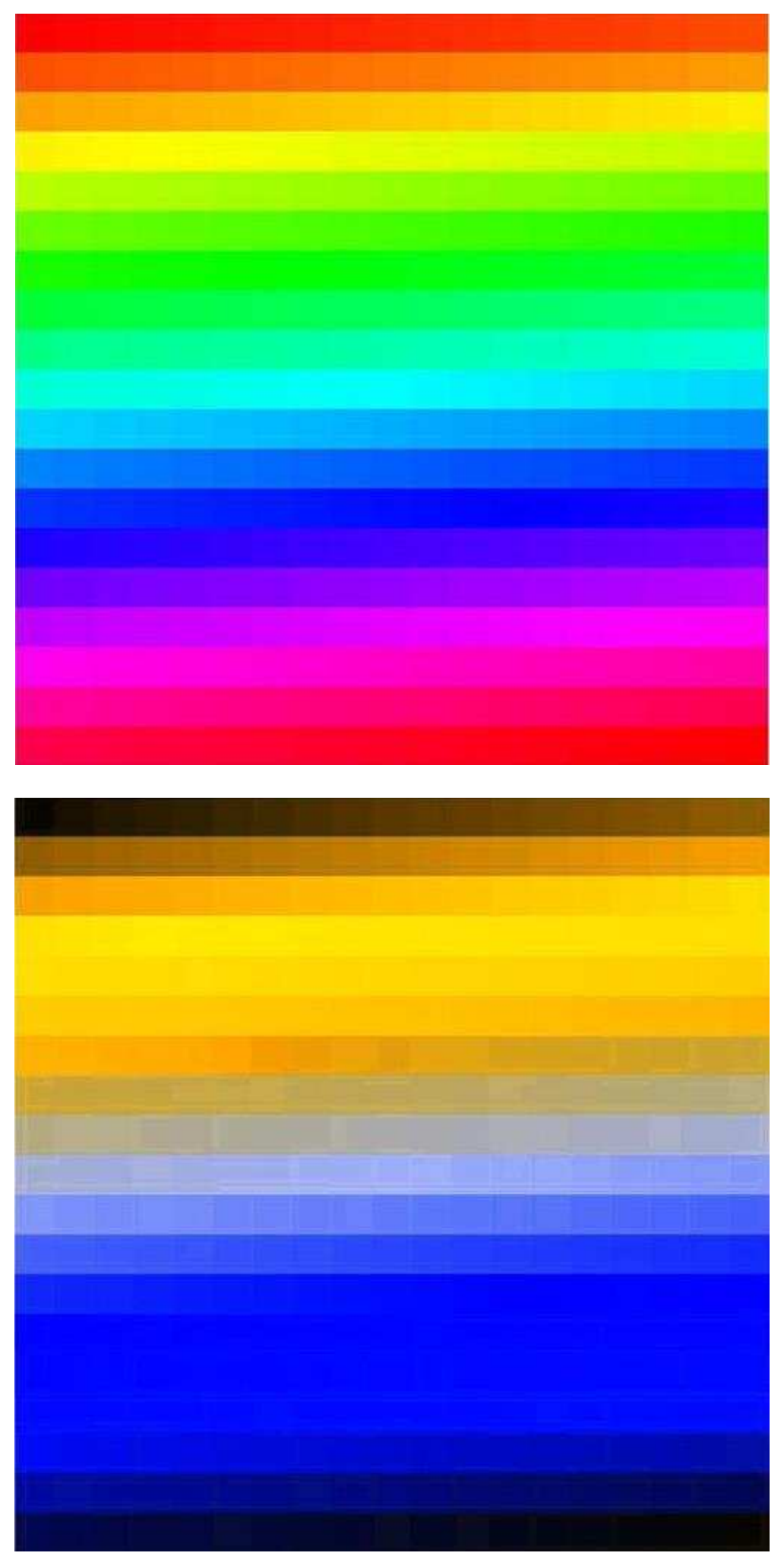

Figure 3.3: (top) Original color card. (bottom) Protanope simulation of the same color card 
This technique also makes our method highly customizable. Although we are working on a case of protanopia, exact same code can be used to recognize the safe and troublesome hue range of a deuteranope or tritranope patient. The only thing that needs to be changed is comparing the original image with the simulated image of that particular type of CVD. Another interesting advantage of this technique is the fact that we can use the method presented by D.R. Flata et al. [2] to create a personalized simulation of how a specific patient sees our created color card and calculate the exact safe range of hues for that particular patient. This method will be explained briefly as a doorway to our future work in Chapter 5.

The marked areas in the Figure 3.4 demonstrate the range of hues that are perceived almost identically by a viewer with normal color vision and a protanope patient. The threshold has been set to 6 degrees in our code which gives us almost the same range as we can detect ourselves manually. However, smaller threshold gives smaller safe range which despite making the accuracy higher, makes the transformation a lot more limited and complicated.

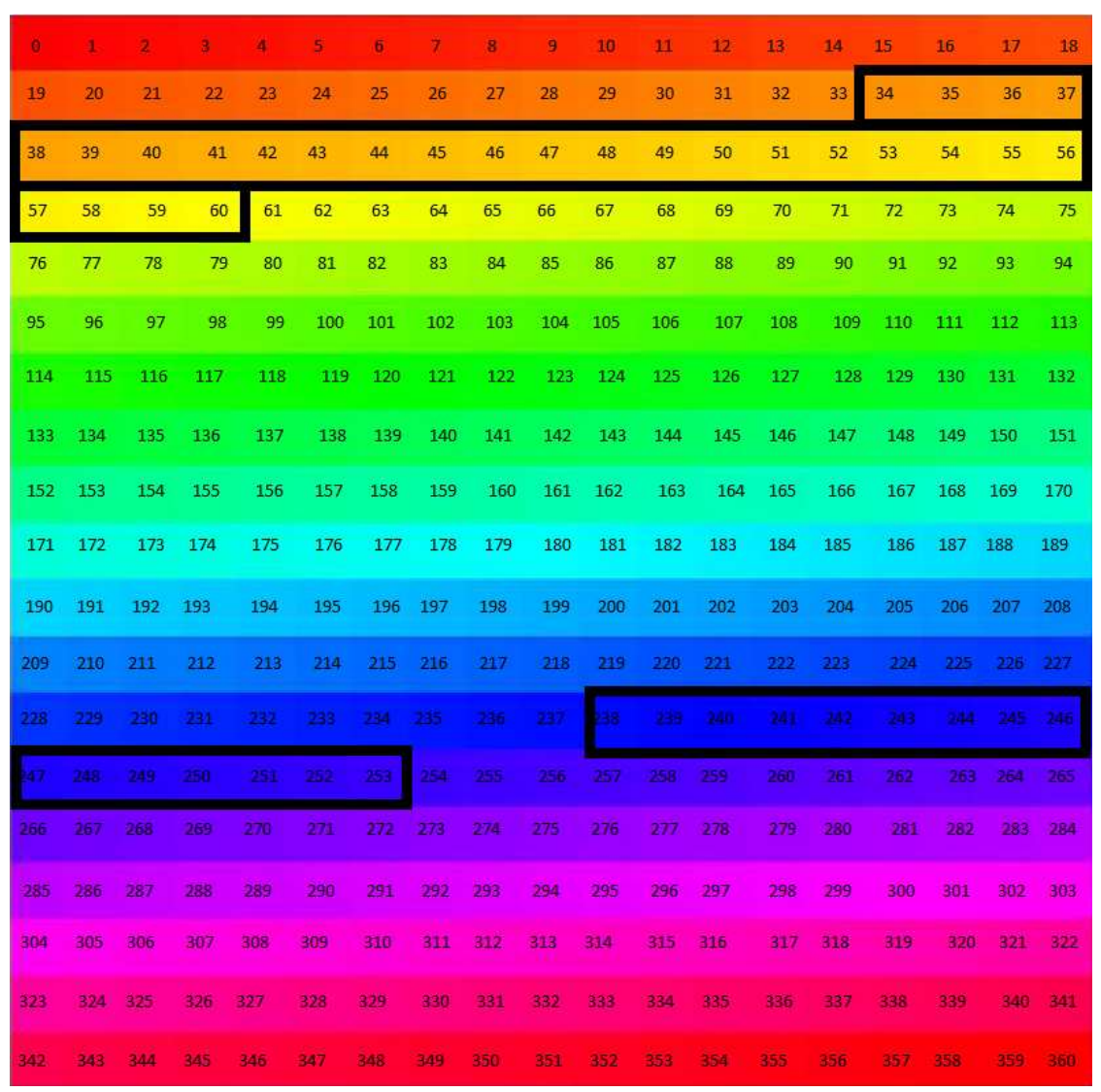

Figure 3.4: The selected hue range correspond the hue range which is perceived identically by normal and protanope visions. We call this range, safe hue range. 
Detecting these "safe hue ranges" is very important simply because it assures us that there is no information loss as long as the color of a section of our digital image falls into these ranges. If a normal vision can detect a difference between two colors in this safe zone, we can make sure a defective color vision can also recognize this difference. Therefore, we do not need to change the colors which originally lie in this range ${ }^{1}$. However, as we can see in Figure 3.4, these safe zones are covering a small range of the whole spectrum. What should we do about the rest of the hues which usually make confusion for our color blind patient?

In order to answer this question we have to go back to our initial goal. As we repeated over and over, we aim to increase color discriminability of our dichromatic patient so that if there are parts of the original picture in which some details cannot be perceived, after our color transformation, they can be perceived. Therefore, our next step is to discover which hue ranges (which are perceived as totally different colors by a normal color vision) end up being perceived roughly the same by a dichromatic patient. The significance of this step can be boldly expressed in Figure 3.5. This is one of the usual hardships color blinds deal with on a daily basis.
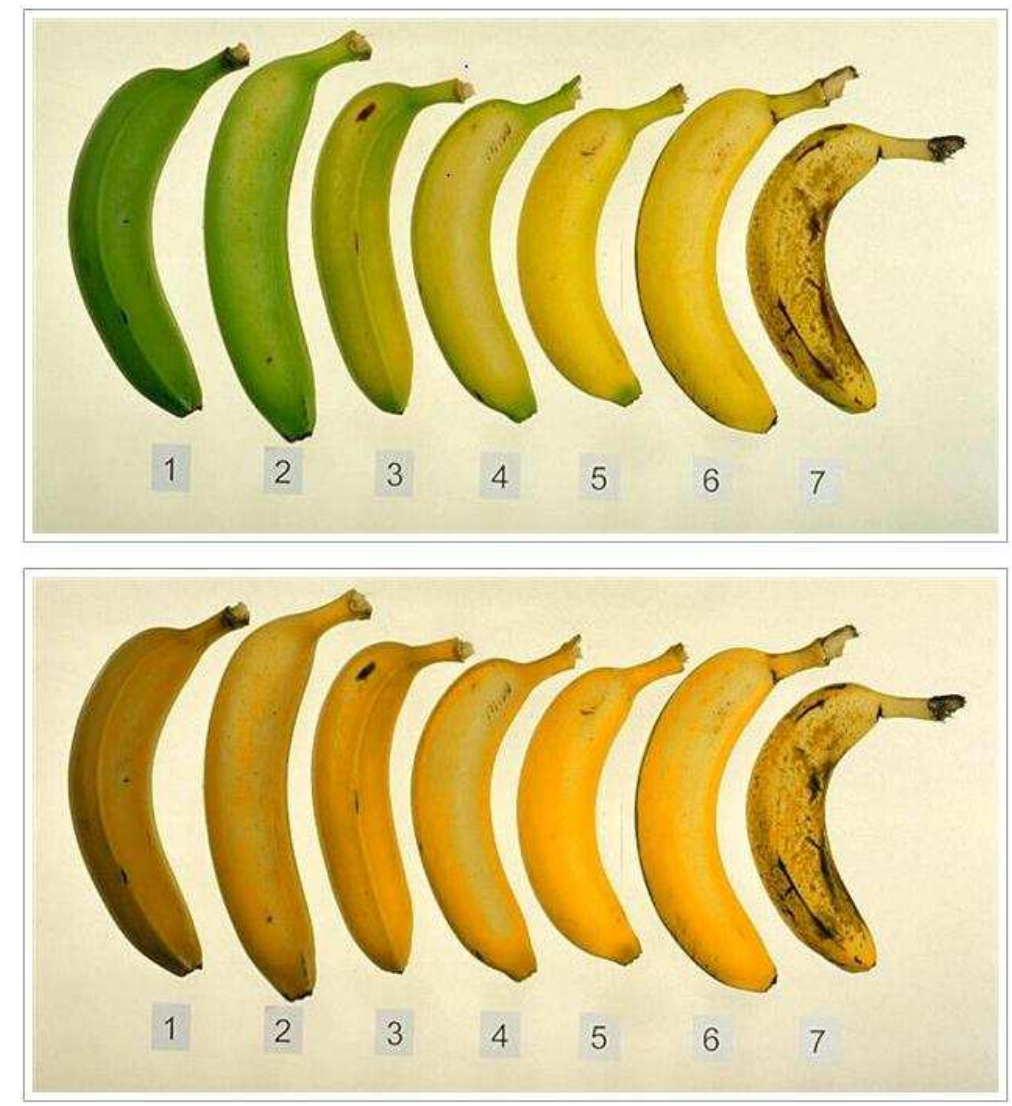

Figure 3.5: A collection of raw and ripe bananas as perceived by (top) normal vision (bottom) protanope vision

\footnotetext{
${ }^{1}$ There is a small process we need to apply on colors of this range to prevent further confusion. We will discuss this step later on in this chapter.
} 
As we can see in Figure 3.5, both ripe and raw bananas are perceived as shades of yellow so differentiating them on a digital image might prove to be a problem for a dichromat. Based on Figures 3.3 and 3.4 we can categorize the entire hue range into three different groups each of which will need special considerations during our color transformation algorithm:

First group is those hues which are perceived dramatically different by dichromatic patients. Sometimes even shades of the same color which might end up being perceived completely different can be included in this category. A clear example is the family of red colors. By looking at Figure 3.3 we can see the red hues between 0 and 28 are perceived as brown and dark brown while other shades of red hues between 342 and 360 are perceived as dark blue and black. Obviously, if there is discriminable difference between two colors in the original picture, it is only suitable if our converted picture has the same discriminable different as well. The opposite is also true. When two colors are roughly the same, they should not be perceived dramatically different. This is why we have to do some sort of color transformation method on colors belonging to this category. For more convenience in the next section we call colors belonging to this group, troublesome colors.

Second group is the group of colors which we already introduced as safe colors. In case of protanopia we can see that our safe colors include shades of yellow and blue. As we explained earlier, colors belonging to this group are perceived identically by normal and defective color vision. Therefore, we try our best to maneuver around these hue ranges. Obviously, there is no need for any hue change as any detail in the colors lying in this category is easily detectable for our dichromatic patient. However, there is a consideration to prevent confusion between transformed colors and colors that were originally in the hues that we often use as our transformation destination. Later on this chapter, we will discuss how we prevent this sort of confusion by altering saturations.

Finally our last group of colors consists of those hues which are perceived differently but are close to our safe colors. We call the colors in this range self-correcting colors. A clear example of colors in this category is hue range of 61-80. While we are trying to eliminate confusion in red section 0-28 we might end up being transformed into this range. Although this range is not among our safe colors but the way they are perceived is close enough to the colors of second group. Therefore, the patient will not have any confusion perceiving them. Again some considerations need to be taken in order to prevent post-transformation confusion for colors in this group.

In the next section we will explain our transformation algorithm in detail. Although we will show some results to clarify how the algorithm works, more results are presented while comparing our method with similar alternative methods in Chapter 4. In Chapter 5, we will exclusively present some more examples as well as the result of our tests on the actual color blinds. 


\subsubsection{Color Transformation Algorithm}

Based on everything we talked about so far, we can now implement our color transformation algorithm. Color transformation means that we transform the existing color space of an image into another color space in which colors are distinguished better by our color vision deficient user. This does not expand the visual spectrum of such users; only utilize their limited spectrum in a more suitable way. The basic rule set we followed during the implementation of our algorithm is as follows:

Rule 1: We reserve the maximum saturation (one) only for the transformed colors. This way if some part of our image has a color originally in the same hue as our transformed color, they will not be perceived the same way and possible confusion will be prevented.

Rule 2: The colors that lie in the hue range which we use as our destination for some color transformations need to be dealt with as well. We reach this goal by altering the saturation of such colors. Saturation value of 0.5 is reserved for this range of hues.

Rule 3: Some colors can be left alone to be perceived the same way that they are perceived everyday by our dichromatic patients. Such colors are exactly where we want them to be so we leave them alone.

Rule 4: Colors such as White (saturation $=0$ and intensity $=1$ ) and Black (Intensity $=0$ ) should stay unchanged. In order to reach this goal we put a condition to prevent such colors from any color transformation as they can be perceived perfectly fine by our color blind user.

By applying these set of rules, we can transform our original image into an image which can be perceived with far more details by our dichromatic patient comparing to the original image. This is sometimes achieved by shifting the hues to a safer hue range which prevents confusion for some colors and sometimes by changing the saturation for others. As we will see the transformed pictures might not look natural to a normal color vision but they will still fulfill our intended purpose. We wanted to improve the information perception from a confusing image and this algorithm will perform that for us. Once again, this algorithm needs to be looked at as a tool to eliminate confusion. Therefore, if a picture is perceived with details as it is, there is no need to perform our algorithm as color transformation might make changes which make the picture look unnatural. In the remainder of this chapter we will emphasize on the pictures that are typically making confusion for dichromats and show how our algorithm can eliminate this confusion and improve details perceived from those pictures.

Our first example is shown in Figure 3.6. The top-left image is the original image which is the flag of Wales. It is a perfect example of how having green and red colors in the same image can 
lead to confusion. The bottom-left image shows a protanope simulation of the same picture. The simulation shows that this image will be perceived as shades of black and brown which makes it very hard to perceive details that a person with normal color vision recognizes. The top-right image is the result of applying our method on the original image and the bottom-right is the simulation of transformed image. Although the transformed image might look weird to a normal viewer, it can be perceived with a lot of details by a protanope.
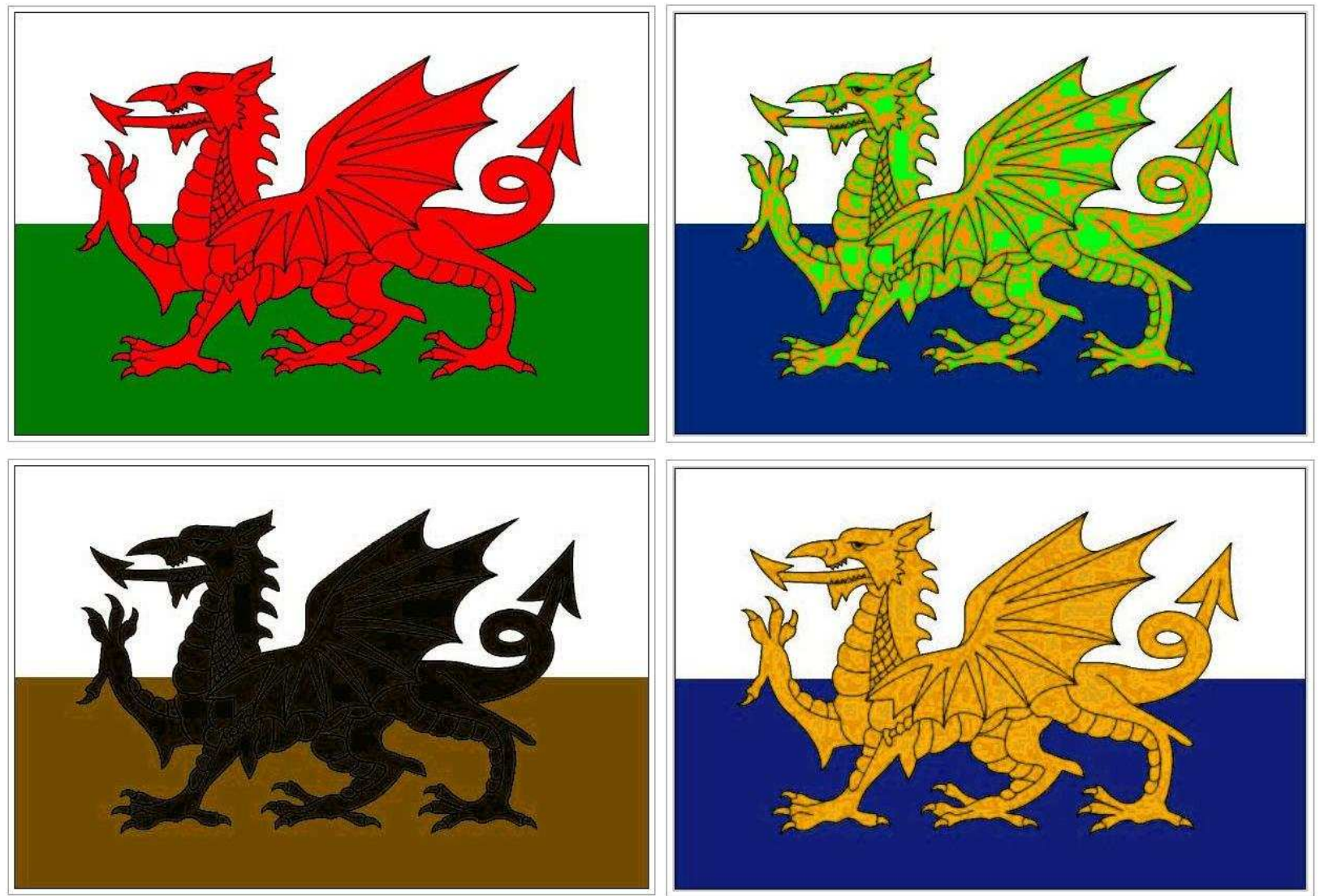

Figure 3.6: (top-left) The original image (top-right) The transformed image (bottom-left) Protanope simulation of the original image (bottom-right) Protanope simulation of transformed image

Another good example of how our method can improve the information perception is shown in Figure 3.7. We used this image earlier in this chapter. The two images on the left show the original image and the protanope simulation of it. As we mentioned earlier, the dichromatic patient might not be able to distinguish which banana is ripe and which one is raw. As a matter of fact it might seem like banana number one is so ripe that it has gone bad which is not true.

Two images on the right hand side show the result of application of our method as well as how it is perceived by a dichromat. After we apply our algorithm on this image we can see that our protanope user can distinguish between a ripe banana and a raw one. By giving a simple explanation about how our color transformation method works, the dichromatic patient can 
easily interpret the resulting pictures and perceive details that would otherwise be impossible to perceive.

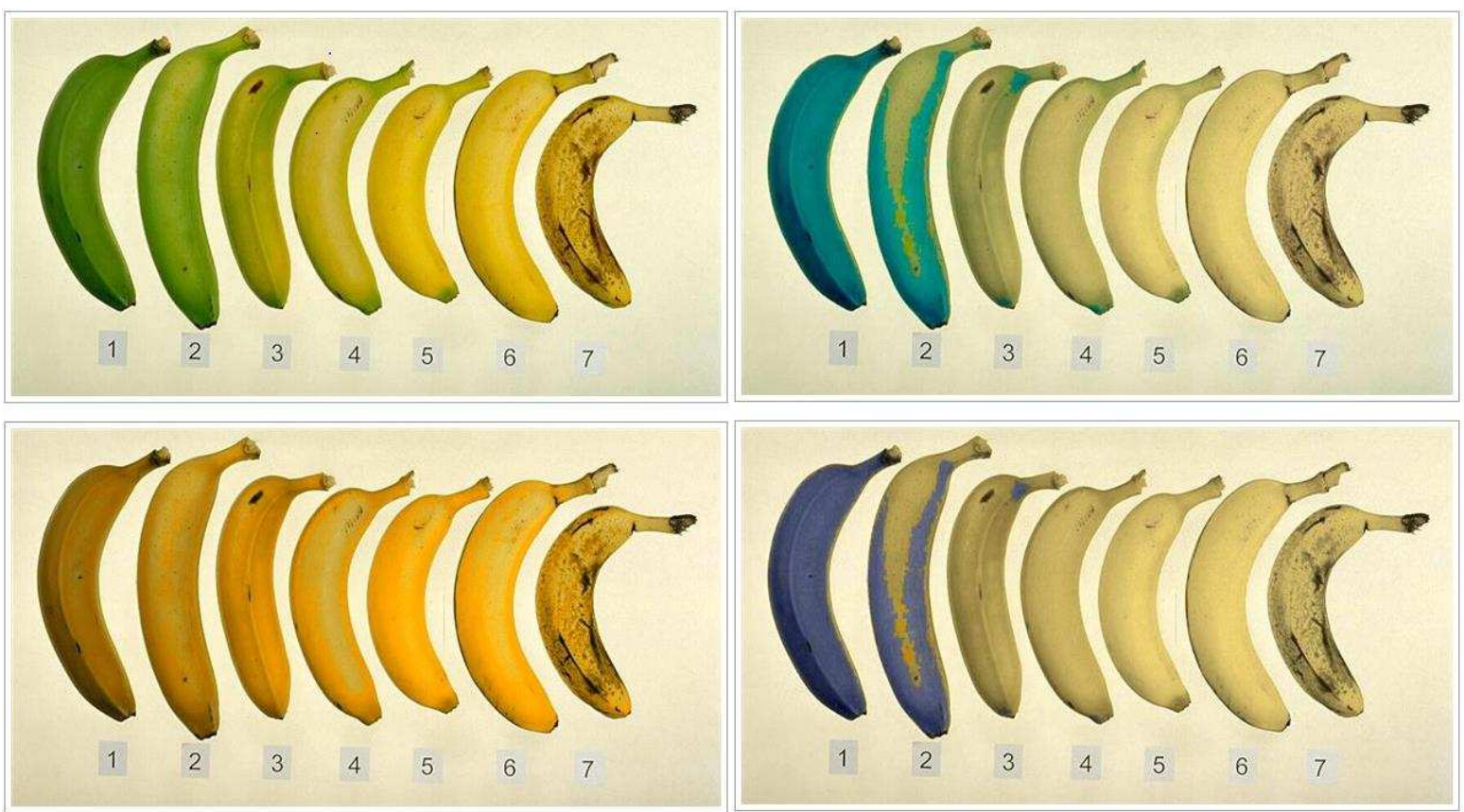

Figure 3.7: (top-left) The original image (top-right) The transformed image (bottom-left) Protanope simulation of the original image (bottom-right) Protanope simulation of transformed image

A noticeable difference between bananas in the left side of Figure 3.7 and those on the right side is that yellow bananas on the right side are a bit dimmer than pure yellow bananas on the left. This is happening because of our confusion-eliminating rule of changing the saturation. As we explained we need to make sure the viewer can distinguish between a color which used to be originally yellow and a color that has transformed from another color, say red, into yellow. This is done by changing the saturation as we can see in this image.

Another note to pick up is the fact that only bananas number 1 and 2 and partially banana number 3 have been subjected to color transformation. This brings up one of the advantages of our method which is its customizability. Our algorithm has been circling around the numbers that we deduced through our very own color card matching presented in Section 3.4.2. Although it is done for a protanope user it can be generalized to other types of dichromacy or it can be even customized to have different sensitivity for example more sensitivity towards a certain color like green. It is only a matter of how we do the hue shift and saturation alteration. Our default values are set to give the best result for the largest group of protanopia and we have tried to make all colors that typically look the same to them, be perceived totally different so that they can sense the difference between colors and can perceive more details from a certain image. 


\subsubsection{Converting HSI back to RGB}

To summarize what has been done so far, we started by reading an image which was initially in RGB color space. The RGB format does not give us the necessary facilities to perform our color transformation method freely. That is why for each pixel of the picture, we perform an RGB to HSI conversion. Once we have our image in the ideal HSI format, we start applying our customized color transformation method on each and every pixel of the picture. During this transformation, our code follows specific set of rules we set in order to prevent further confusion and also avoid changing of harmless colors of the picture.

Once all the steps above are done, we end up with a picture in HSI color format with transformed colors. In order to display the picture, it has to go back to the original RGB format once more. Suppose we have HSI values of each pixel in the interval $[0,1]$. In order to find the corresponding RGB values in the same range, we first multiply $\mathrm{H}$ by 360 . This operation returns hue to its original range of $[0,360]$ degrees. Now based on the resulting hue we will have three different cases [3]:

1) RG sector $(0 \leq \mathrm{H}<120)$ : When the hue of our pixel lies between red and green sections:

$$
\begin{aligned}
& \mathrm{B}=\mathrm{I}(1-\mathrm{S}) \\
& \mathrm{R}=\mathrm{I}\left[1+\frac{S \cos H}{\cos (60-H)}\right] \\
& \mathrm{G}=3 \mathrm{I}-(\mathrm{R}+\mathrm{B})
\end{aligned}
$$

2) GB sector $(120 \leq \mathrm{H}<240)$ : When the hue of our pixel lies between green and blue sections; In this case we first subtract 120 from our hue $(\mathrm{H}=\mathrm{H}-120)$ then:

$$
\begin{aligned}
& \mathrm{R}=\mathrm{I}(1-\mathrm{S}) \\
& \mathrm{G}=\mathrm{I}\left[1+\frac{S \cos H}{\cos (60-H)}\right] \\
& \mathrm{B}=3 \mathrm{I}-(\mathrm{R}+\mathrm{G})
\end{aligned}
$$

3) BR sector ( $240 \leq \mathrm{H} \leq 360$ ): When the hue of our pixel lies between green and blue sections; In this case we first subtract 240 from our hue $(\mathrm{H}=\mathrm{H}-240)$ then:

$$
\begin{aligned}
& \mathrm{G}=\mathrm{I}(1-\mathrm{S}) \\
& \mathrm{B}=\mathrm{I}\left[1+\frac{S \cos H}{\cos (60-H)}\right] \\
& \mathrm{R}=3 \mathrm{I}-(\mathrm{G}+\mathrm{B})
\end{aligned}
$$




\subsection{Passing Different Tests}

Now that we explained the process of our color transformation method, we start delving into more details and showing different examples where different scenarios of color composition might happen in an image. In section 1.3 we briefly mentioned color vision tests. Pseudoisochromatic screening test plates prove to be the most popular and applicable among them [34].

Pseudoisochromatic (PIC) plates are delicate examples of color camouflage. The hidden object and the background of the test design are colored spots or patches of random size and luminance [34]. The colors of the background and the actual test object are masterfully aligned along the dichromatic confusion line ${ }^{1}$, with variations of luminance and chromatic saturation [35].

In the first example in this section we chose one of the Ishihara color blindness test plates which exploits how red color is perceived by protanope patients. As we saw in Figure 3.3, shades of red color close to zero degree hue are perceived as dark brown or even black. This is exactly why this could be a good test to distinguish whether an individual is protanope or not. The left image in Figure 3.8 shows the original test image. Extracted version of the object is presented in the middle and on the right hand side we can see a simulation of how this image is perceived by a protanope.
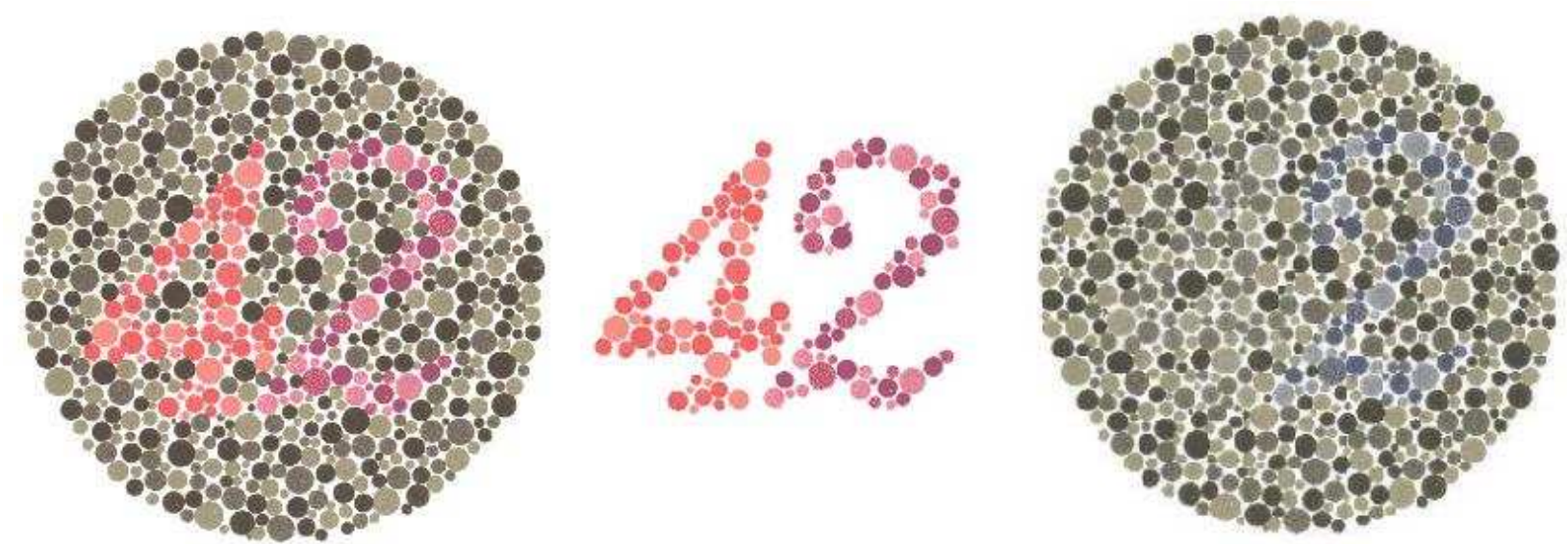

Figure 3.8: (left) Original Image (middle) Extracted object from background (right) Protanope Simulation

As we can see, number 42 is easily distinguishable by a person with normal color vision. However, an individual who has protanopia, as illustrated in Figure 3.8 (right), cannot perceive number 4 at all and barely perceives number 2 . Now let us apply our color transformation

\footnotetext{
${ }^{1}$ Confusion lines represent the series of narrow zones in the CIE $\mathrm{x}, \mathrm{y}$ in which a person with dichromatic vision perceives colors identically [35].
} 
method on this image and see the result. In Figure 3.9 we put the original image on top left, our transformed image on top right, the protanope simulation of the original image on bottom left and finally the protanope simulation of our transformed image on the bottom right of the image.
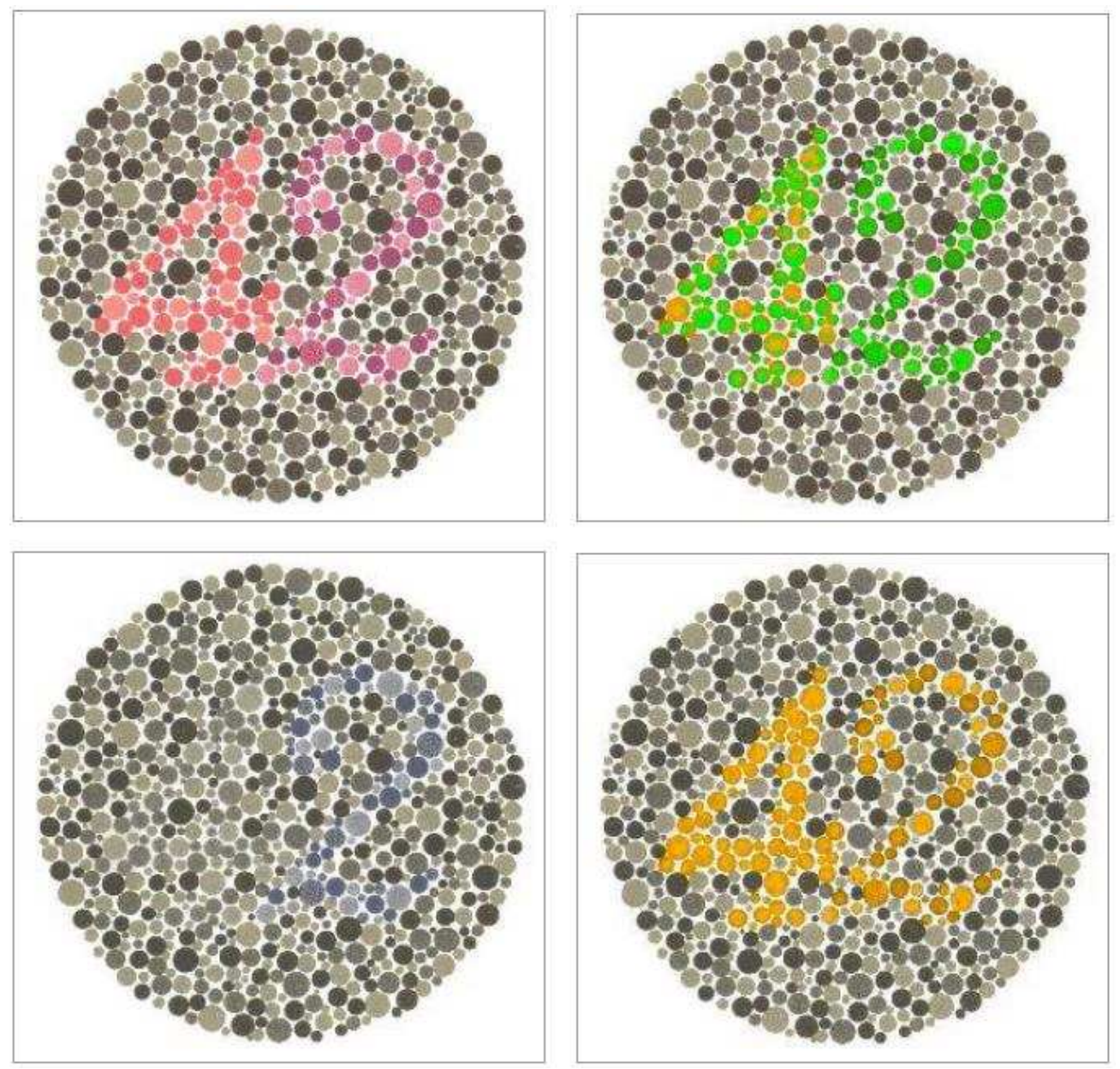

Figure 3.9: (top-left) The original image (top-right) The transformed image (bottom-left) Protanope simulation of the original image (bottom-right) Protanope simulation of transformed image

This example is another indication of how our color transformation method improves information perception for users with dichromatic color vision. Tests of such kind have been designed specifically to challenge the color blinds. If our method can help them pass these tests, we can rest assured similar cases which probably have less complexity, won't be an issue.

Another interesting fact in this image is to watch how our rules come to life. Let us check those rules one by one. In our transformed image we can see that different shades of red and purple are transformed into shades of orange and green but fully saturated (rule 1). The intensity of pixels and the distance between colors are preserved and dots that were darker in the original image still have their low intensity. We can also see that because of our forth rule, the black and white colors are preserved perfectly. Since we do not manipulate intensity in our algorithm, we can say 
very low intensity pixels are self-preserved. Figure 3.9 is a good example of such a case. In this image, even if the dark dots have a red hue for example, no problem will arise. Even if hue transformation happens, since intensity is kept constant, the dot will still appear as black.

This however, is not the case for low saturation pixels. The background of Figure 3.7 is a perfect example of such situations. Background pixels in that image have very low saturated red hue with high intensity. If we had not considered rule 4, all background pixel would be subjected to hue transformation and this time since the intensity is high, it would be completely obvious that something has gone wrong. That is why before each one of our condition tests we have to first check if the saturation of our pixel is above a certain threshold or not.

The next group of images in Ishihara test plates revolves around how a specific hue range of 3334 degrees are perceived. Figure 3.19 shows an example of this type of plates. As we can see our color transformation method do not manipulate the dots in the background as their color is not among troublesome colors.
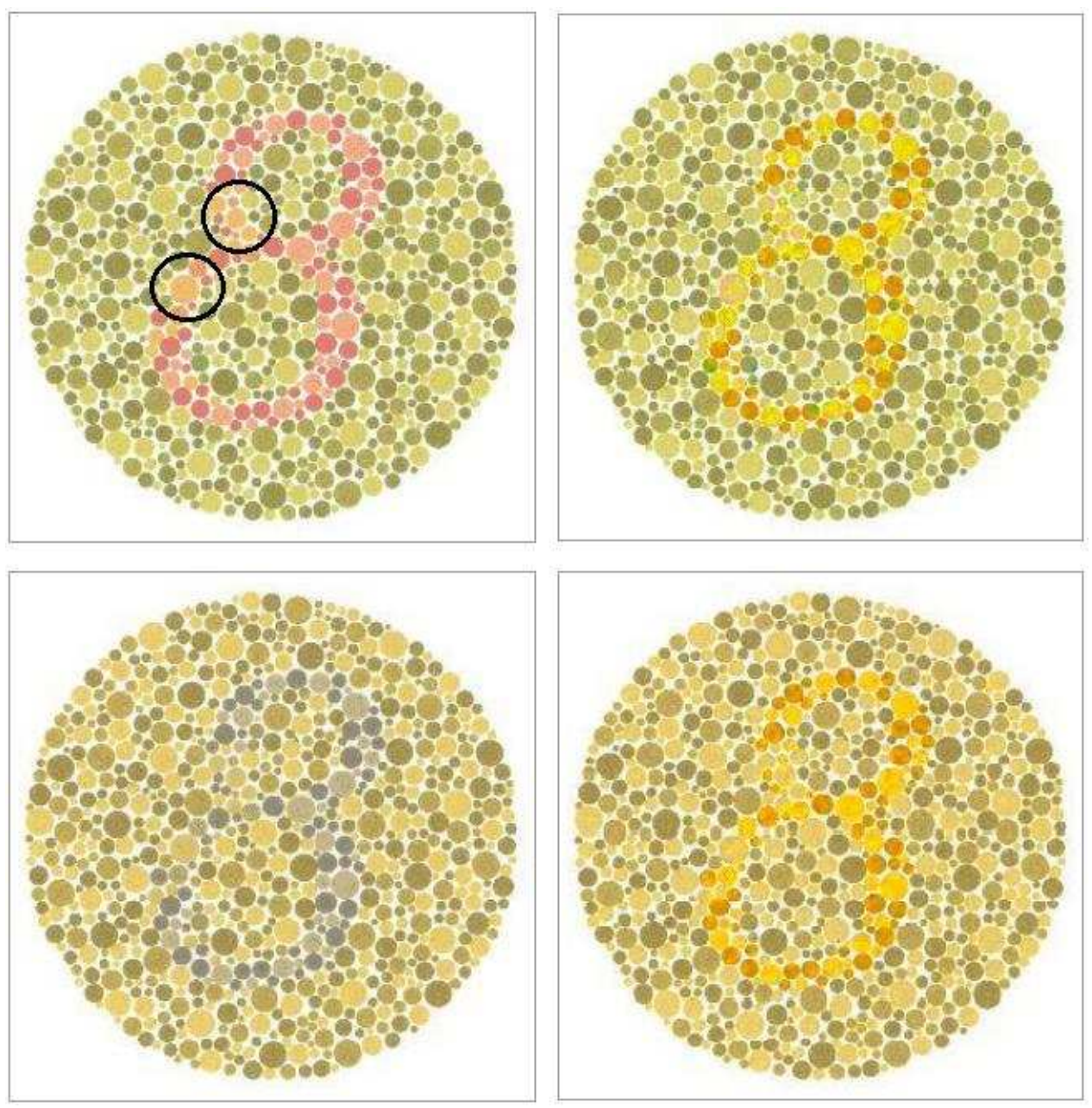

Figure 3.10: (top-left) The original image (top-right) The transformed image (bottom-left) Protanope simulation of the original image (bottom-right) Protanope simulation of transformed image 
The most important parts of Figure 3.10 are the two highlighted regions. These two regions contain dots whose hues are around 33 to 34 degrees. This range is exactly on the border of our safe zone. The test has masterfully put dots in those regions in such hues that will be perceived exactly the same as how the background would look like. That is why a protanope user will detect number 3 in that image. Fortunately, our approach changes the colors of the dots in such a way that number 8 pops up and users will most likely get the answer right.

Some of the other Ishihara test plates have color composition similar to Figure 3.11. As we can see the patient will barely see anything from the original image. In the best case, our protanope user might wrongfully detect number 2. Our color transformation method changes the appearance of the image in such a way that detecting number 5 would be an easy task after the conversion.
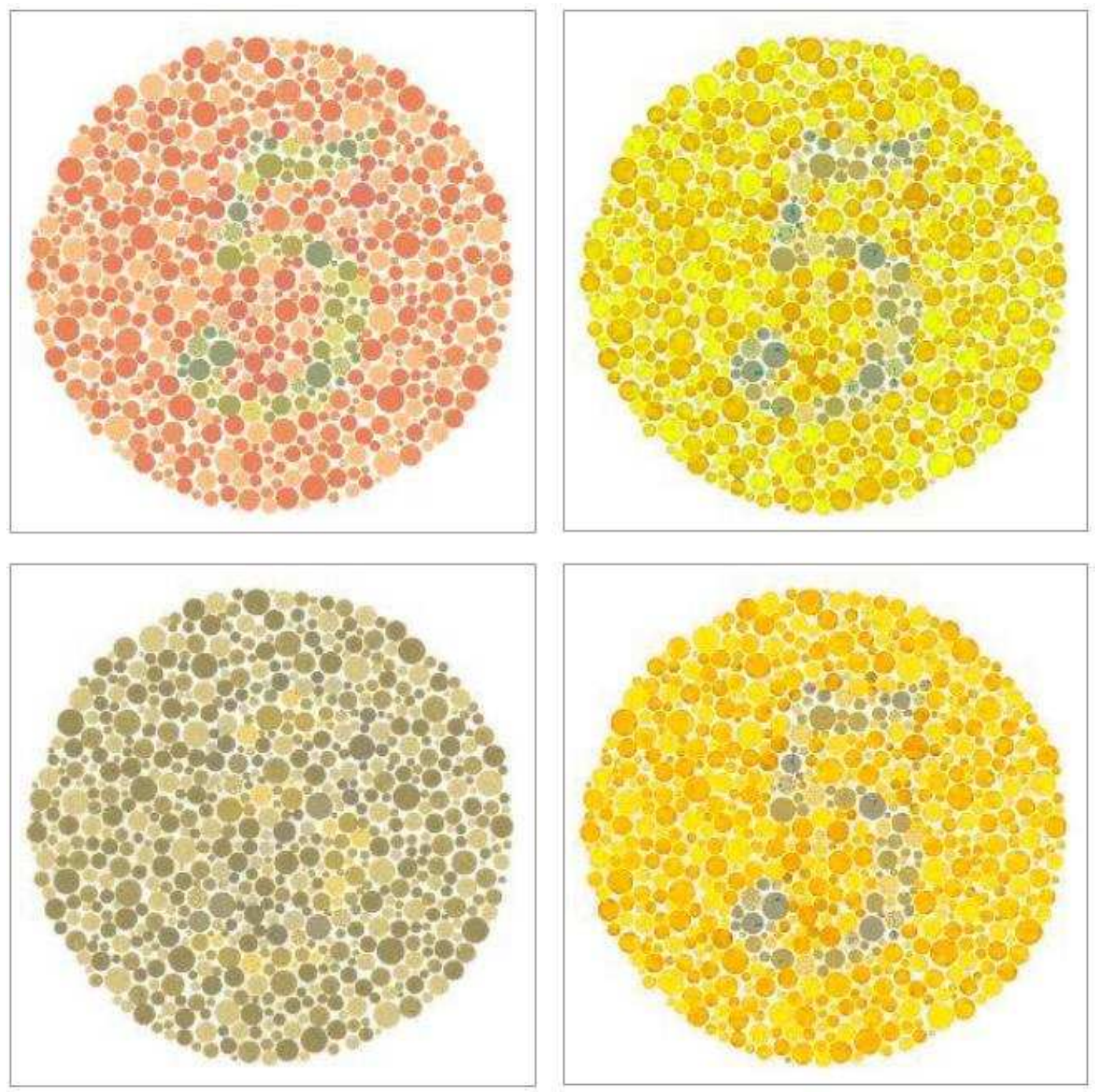

Figure 3.11: (top-left) The original image (top-right) The transformed image (bottom-left) Protanope simulation of the original image (bottom-right) Protanope simulation of transformed image 
Another clever design is the test plate with number 74 hidden in it. A protanope user will see that as number 21 or 71 . Our method can improve this perception and help them find the hidden 74 most likely. This image is shown in Figure 3.12.
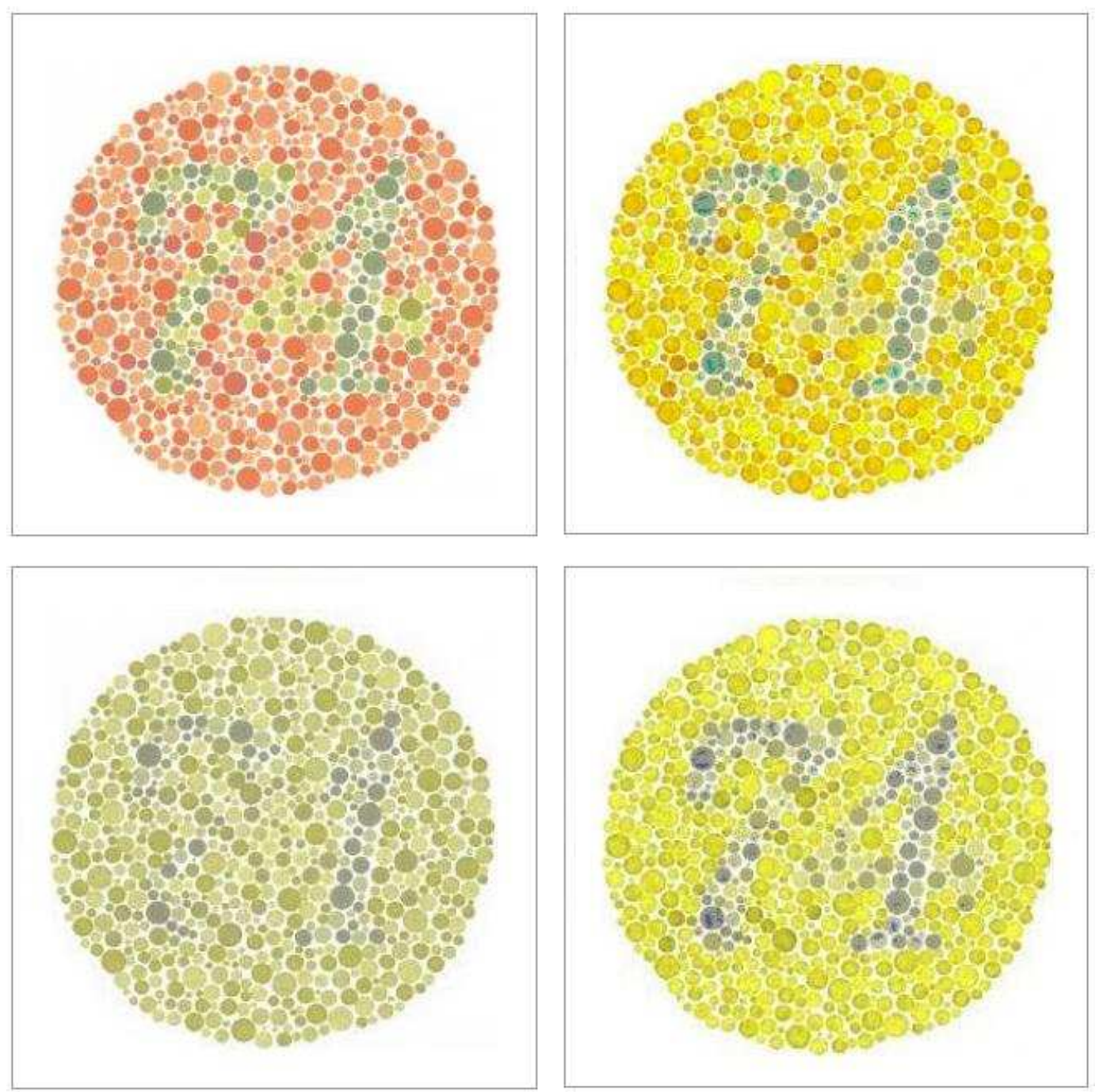

Figure 3.12: (top-left) The original image (top-right) The transformed image (bottom-left) Protanope simulation of the original image (bottom-right) Protanope simulation of transformed image

Another group of Ishihara plates are those which have been specifically designed to detect color blindness in general. Although detecting the hidden pattern in such test images seem like a trivial task for people with normal color vision, the majority of color blind population will see nothing popping up in the middle of the dot pool. Figure 3.13 is a clear example of such test images. In this specific test, number 6 will not be recognizable to our color deficient user as the simulation shows. 

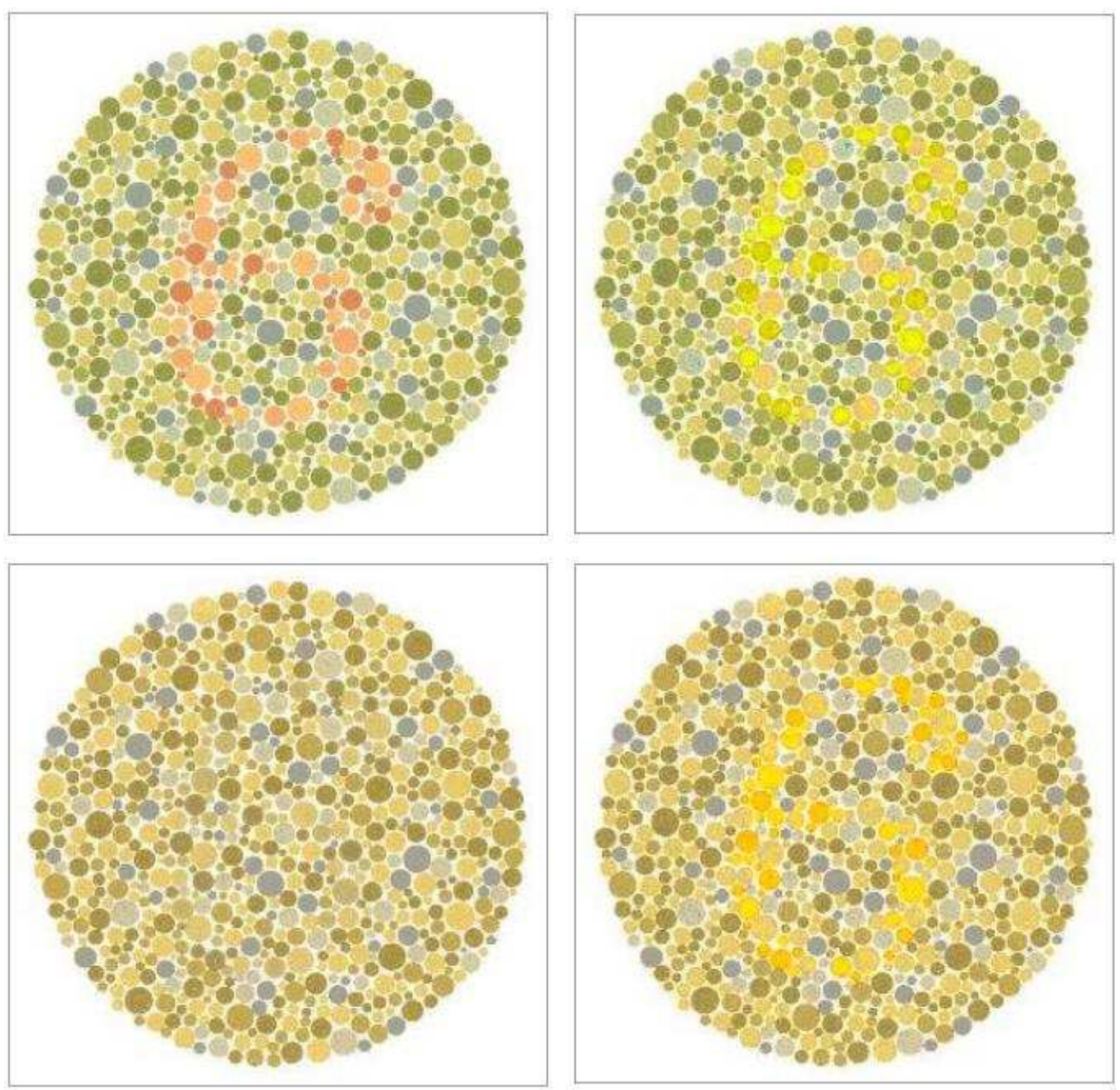

Figure 3.13: (top-left) The original image (top-right) The transformed image (bottom-left) Protanope simulation of the original image (bottom-right) Protanope simulation of transformed image

The hidden pattern is not always a number. A number of pseudoisochromatic screening test challenge the user to follow a line as shown in Figure 3.14. In this image a path is drawn using dots colored by shades of red and dots colored by shades of purple. Theoretically an individual with protanopia should not see the red line as it should vanish into the black background and also only see the blue component of the color purple because of the missing red cone. The simulation proves this theory and we can see that only the purple line will be perceived as a blue line by our color deficient user. When we perform our color transformation algorithm on this image, even the red line can be distinguished by our user which again is an improvement in information perception. 

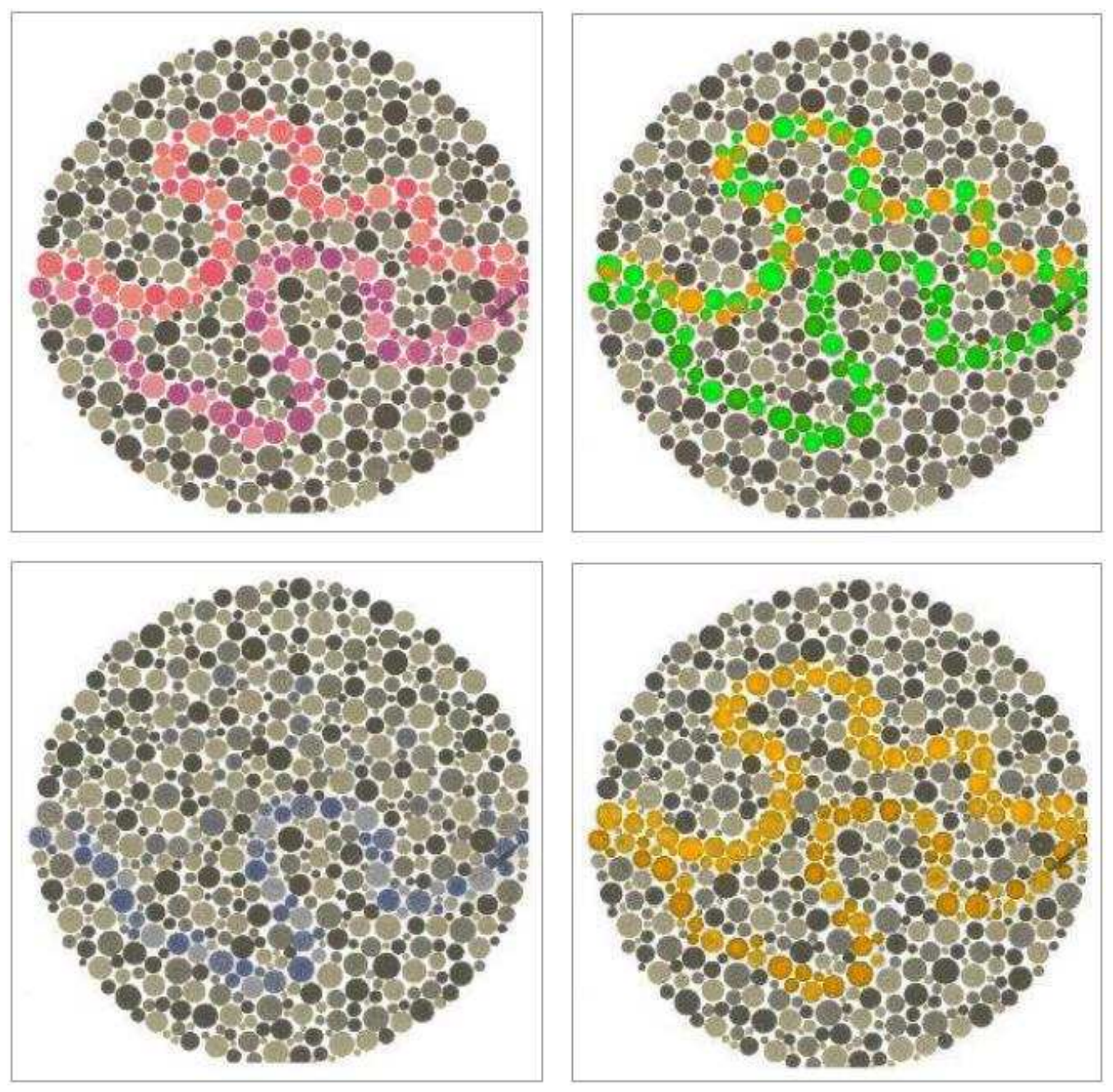

Figure 3.14: (top-left) The original image (top-right) The transformed image (bottom-left) Protanope simulation of the original image (bottom-right) Protanope simulation of transformed image

\subsection{Other Challenges}

The majority of pseudoisochromatic plates like the ones we presented in the previous section have a unanimous goal; they go to the extreme cases to detect type and severity of color vision deficiencies. As we could see in numerous examples in section 3.5, most of these plates exploit colors which would be perceived identically by some patients. Although these tests might be the hardest cases for a color blind to pass, they were not the only challenges ahead of us.

One of the facts that we had to consider during our method promotion was preventing confusion after the color transformation is done. Although this case might not happen that often, we had to cover all the bases. To clarify the problem, when we transform a color with troublesome hue into 
a safer hue there is always the risk that there would be a color with exact same hue in the original image. As we briefly explained before, we eliminate this problem by manipulating the saturation of the transforming colors and the original colors which happen to be in the same hues as the transforming colors. The best way to show this process is by looking at Figure 3.15.

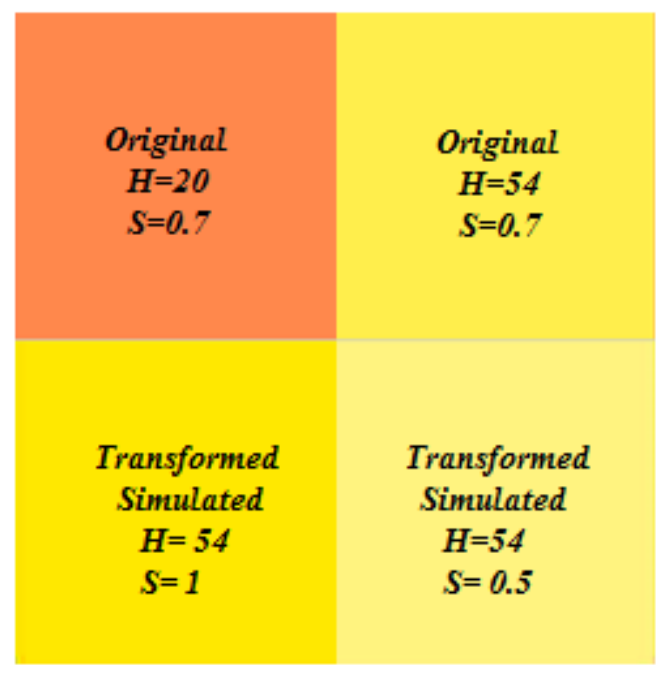

Figure 3.15: Customized color card for post-process color appearance

Let us talk a bit about what the presented picture actually stands for. On the top left of the image we have a dark orange color with hue of 20 and saturation of 0.7 . This hue is one of the hues that typically generate confusion and is perceived very differently. Therefore our algorithm automatically transforms it into another hue which can improve the information perception. The destination hue would be 54 and we show this hue with same saturation (0.7) in the top right section of the image. If we had not introduced our first and second rules about saturation manipulation, these two squares would have ended up with exactly the same color. This however is not the case.

On the bottom half of the image we can see the simulated version of the two sections on the top. We can see that the difference between bottom right and bottom left sections is completely distinguishable. Here is how the algorithm works in such a situation. The dark orange color (hue 20) which is our troublesome color gets shifted into a new hue (54) but the saturation changes into maximum saturation of one, creating the bottom left section. On the other hand, the top right section which originally has a hue of 54 with saturation 0.7 faces a decreased saturation to 0.5 resulting in bottom right section of the image. Due to this trick, we made sure that even if in a very rare scenario, the image already contains one of the hue ranges that we need for transformation, no confusion will arise.

Another challenge we deal with is complicated images like Figure 3.16. Images similar to wales flag in Figure 3.6 are ideal images as they have uniform hue and saturation in each part of the 
image. That results in uniform outcome as well and we will not see color jump in such images. Out in the real world that is not always the case. Figure 3.16 is a clear example of a complicated image with wide range of hues and wide range of saturations in each fruit.
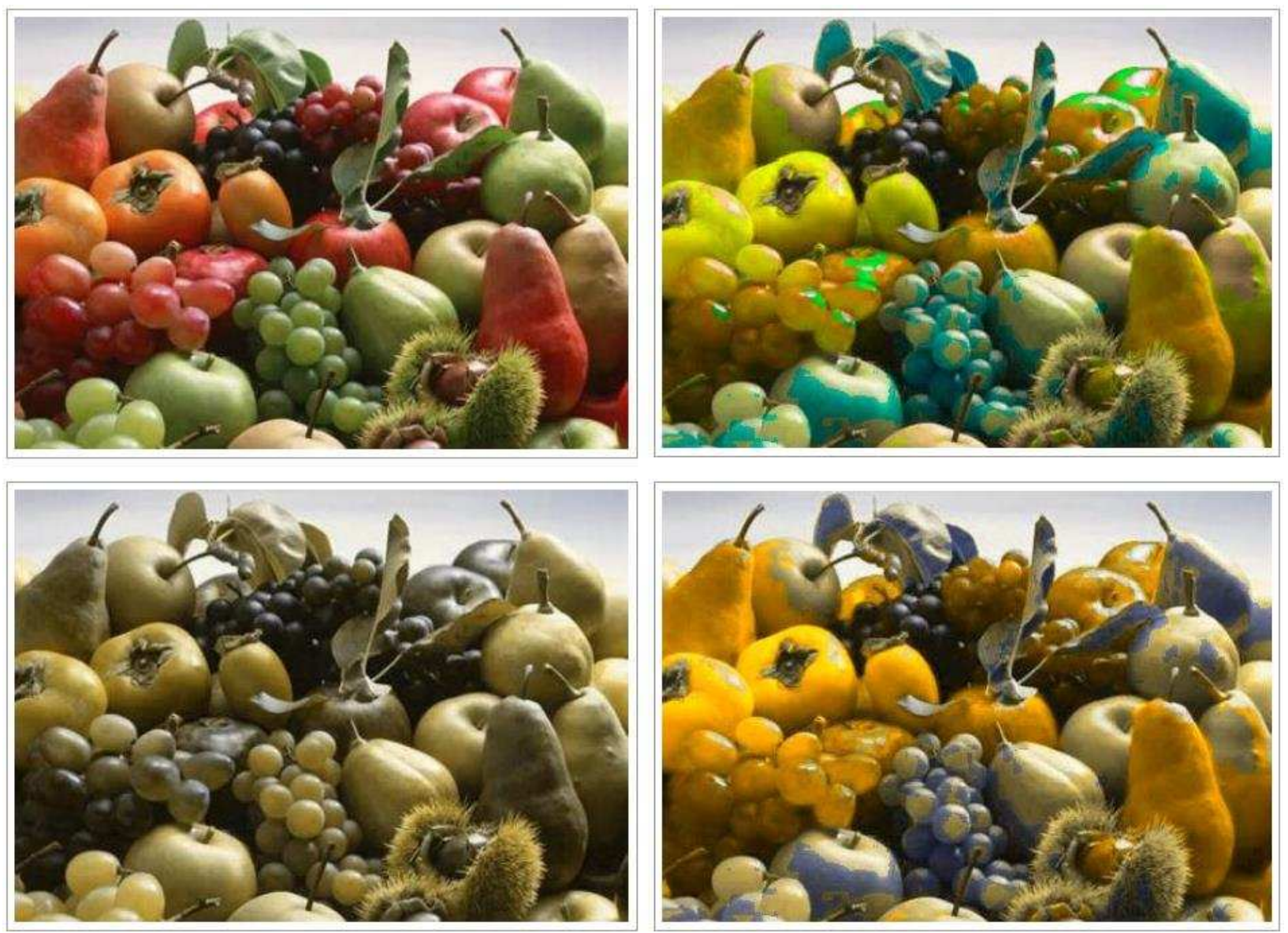

Figure 3.16: (top-left) The original image (top-right) The transformed image (bottom-left) Protanope simulation of the original image (bottom-right) Protanope simulation of transformed image

We can see that even though there are different fruits with various colors in the original image, a dichromatic patient would see a lot of them with the same color. Even though our transformed image might look a bit weird in parts of the image like on the top right pear or on the bottom grapes, it still serves its purpose. As we repeated over and over, our goal is to just improve the information perception. Clearly, our color transformation lets our patient notice that for example there are two different colors of apples or pears in the picture. Green fruits end up with shades of blue and red fruits end up with vivid yellow/orange color. In any case the viewer can easily distinguish different colors in the recolored image. We can also see that even some fruits end up with lively colors in comparison to dismal and dead colors we can see on the simulation of the original image. Overall we can say that even in the worst case scenarios of a complicated image with various hues and saturations, our algorithm gives a successful result. 


\subsection{Summary}

Color vision deficient patients have problem discriminating between certain colors. We introduced a method to recolor the image in such a way that the resulting image ends up giving more information than the original image to our dichromatic patient. This is done by manipulation of hues and saturations.

We categorized the colors into different groups. A group of colors were those which are usually perceived very differently by a CVD patient. Colors like red and green are the most prominent examples. This difference sometimes leads into a total loss of information in a picture as two different colors might appear identically or one color camouflages into background. A shift of hue into another range of hues called safe colors is what we proposed in our algorithm. During this process we sometimes had to also consider changing the saturation of some colors.

A usual problem that people with red-green color vision deficiency face is how they perceive certain colors. Since they view colors like red and green as other colors such as yellow, orange or beige, it is sometimes frustrating for them to know whether a yellow color is actually yellow or it was originally another color but perceived as yellow by them. We fixed this problem by reserving maximum saturation of one, only for transformed colors and saturation 0.5 for the original colors which share the same hue as our destination hues in the transformation process. This way the patient easily can distinguish if a color they see is actually what it originally is or if it is changed by us.

We presented a wide range of examples to show how our method can improve information perception from a digital image for a dichromatic patient. These examples range from simple uniform-hue images to complicated images with wide range of hues, saturations and intensities but what is important here is the fact that all of these images were given to one single code to be recolored. It is not like we use a tailor-made code for each image and perform a specific color transformation for them. That is one of the advantages of this method; it is a stable general approach which works for different color compositions but at the same time it is highly customizable.

Although we worked our way into a uniform code that works for most scenarios, it is only a matter of changing some variables to reach another code which works better for another type of color vision deficiency or for a specific type of image. Suppose we want to recolor a specific colored map for the color blinds. In that case we can easily configure our code in a way that it gives best result for that specific situation. The basics are all the same; just the implementation would be a little bit different.

Another advantage of our method is its speed. A lot of activities have been carried out in the field of recoloring images with the purpose of improving information perception but many of 
them use resource consuming complicated algorithm. Our algorithm however, can recolor the image in a single traversal of all pixels and instantly generate the transformed version. This could be beneficial especially for mobile applications.

In the next chapter we briefly introduce other approaches which try to solve similar problems and try to compare our results with theirs and discuss pros and cons of each method. There might be many different methods but a lot of them share the same principals. That is why we only pick the most distinct approaches and examine their results. 


\section{Chapter 4}

\section{Alternative Methods}

In Chapter 3 we discussed our proposed color transformation method that could potentially improve the amount of information a dichromatic user can perceive from digital images. We used various images to cover different possible color compositions and proved our method works by simulating how the images look like to a dichromatic eye before and after transformation is performed on the images. The real test would be asking actual color vision deficient patients to look at our transformed pictures and see if they perceive the information inside those images better than the original picture or not. This will be presented in the next chapter but for the time being, we want to compare our method with other similar methods.

As we briefly mentioned in Chapter 3, there are numerous activities in progress to improve the quality of life for the people with CVD. Among them one would be recoloring the images in a way that the resulting image can be perceived better by the patient. One approach is our presented method of color transformation. In this chapter we will focus on few other distinct approaches, introducing them, showing their results and if possible comparing their results with our results.

Among the activities we stumbled upon in this field, there were few articles where authors took the same principal as we did for their color transformation. Lai and Chang [23] and Ohkubo and Kobayashi [33] proposed similar methods for using an auxiliary system to enhance visual perception for visually impaired users. They both use hue and saturation shift in their image processing unit to improve the quality of captured images before re-projecting them on the head mounted display. However, the problem is that especially in [23] the authors proposed a simple constant 45 degree shift in the hues. Since the main application of their method would be recognizing red and green traffic lights, that might actually give appealing results in that case but out there in the real world, there are millions of different color compositions and a constant hue shift for every problematic hue in images will not give a good result. Because of this reason and 
also the fact that these articles did not present a picture of their final transformed images, we cannot go into more detail about these two approaches.

\subsection{Self-Organizing Color Transformation}

The first alternative approach we discuss here is the color transformation method proposed by $\mathrm{Yu}$ Ma et al. [5] which utilizes the redundancy of color information and changes the colors of an image into discernible ones for dichromats. This method is based on the fact that the number of colors used in a typical image may be far less than the whole range of color spectrum. In other words, there is plenty of color redundancy in the scene that can be utilized to maximize the color information transferred to dichromats. A nonlinear transformation method is proposed which uses the self-organizing map (SOM) to change the colors. Meanwhile SOM algorithm tries to maintain the neighboring relation between colors as well.

The self-organizing map (SOM) is an artificial neural network which can nonlinearly project the characteristics from a high-dimensional space onto a low-dimensional array of neurons [36, 37]. The SOM can be seen as a vector quantization algorithm for dimension reduction or clustering which uses an unsupervised learning algorithm to cluster input feature vectors [38].

Unlike conventional competition learning algorithms, the weights of the self-organizing map are determined by both the distribution of the input vectors and the topology of the neuron affinity. Consequently, the distance relations of the input feature space are preserved in the mapped space. The training process of the SOM consists of two steps. First, for each input vector, the 'winner' is determined amongst all the SOM neurons. The winner is the neuron whose weight is most similar to the input vector. Second, the weights of both the 'winner' and its neighbors are adjusted to approach the input vector. Yu Ma et al. [5] state two reasons for choosing the SOM algorithm for their color transformation method: First, the nonlinearity enables it to compress the color space better than linear ones. Second, it maintains the neighboring relations between colors. Figure 4.1 illustrates the flowchart of this method.

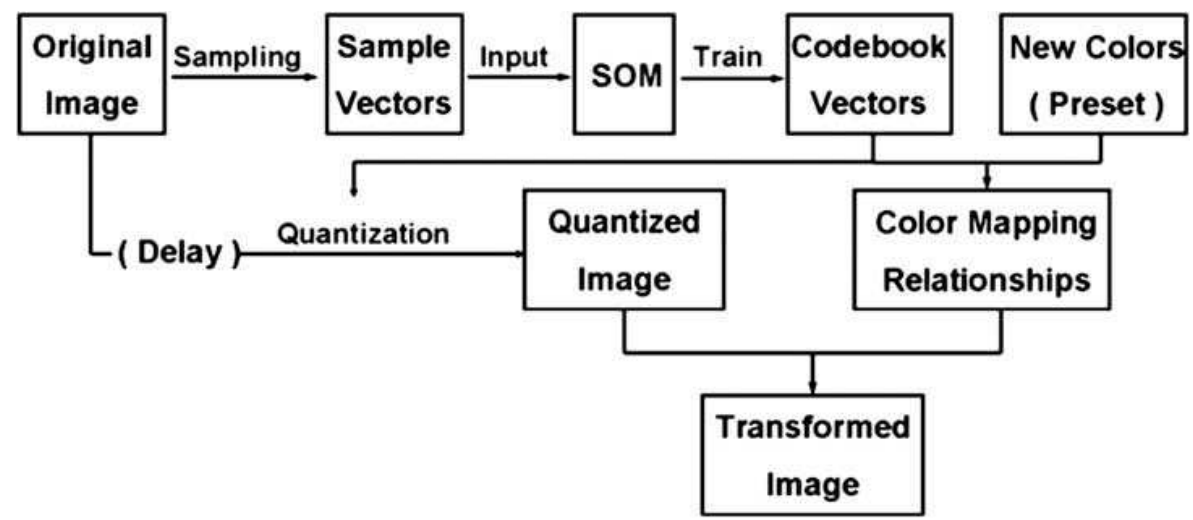

Figure 4.1: The flowchart of self-organizing color transformation method 
In a typical image, adjacent pixels (especially when located in uniform regions) may have similar colors. Because of this repetition, a preliminary sampling for the original image is executed to reduce the computational cost. This sampling is conducted in a random order and the number of samples is determined by the image size. After the self-organizing process, the weights of the SOM network are fixed. They are called "codebook vectors" or "reference vectors". Each reference vector corresponds to one color in the original color space. Meanwhile, a new color space is created with the same size and resolution as the reference color space. It has black and white in two opposing corners, blue and yellow in the other two corners, and a smooth interpolation in between, as shown in Fig. 4.2. In the next step, the reference color space is rotated to make its corner colors most closely related to the corresponding corner colors of the new color space.
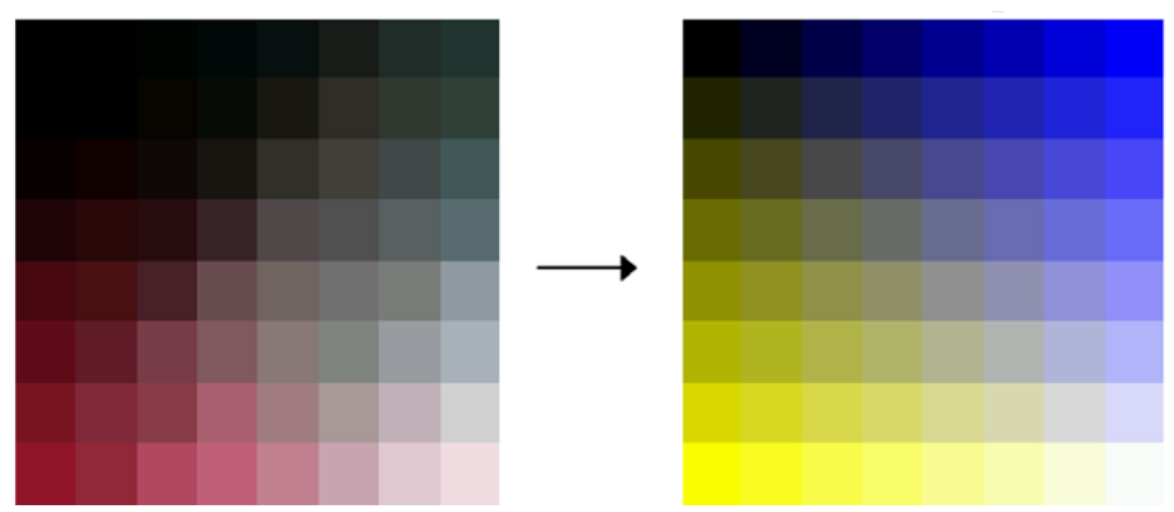

Figure 4.2: (left) The original colors of the reference vector (right) Their mapped colors

The next step is to perform the color transformation on the original image. The feature vector of each pixel (i.e. the RGB values) in the original image is compared with all the reference vectors in order to find the most similar one, whose corresponding color in the new color space is set as the transformed color for the pixel. The transformed image is obtained using this mapping process for each pixel [5].

Figure 4.3(a) shows a large image (2000 pixels by 800 pixels) containing different colors. The protanope simulation of this image is presented in Figure 4.3(b). Authors show four different blocks in Figure 4.3(c) which contain the representative colors in the scene. Figure 4.3(d) illustrates the acquired SOM reference vectors with their corresponding colors after the training. The final result is shown in Figure 4.3(e). The first thing that draws attention in this image is the fact that we did not present a protanope simulation of their final result. That is because, the image transformed by this method is a bi-color image composed of blue and yellow which are perceived perfectly normal by a protanope. 
a

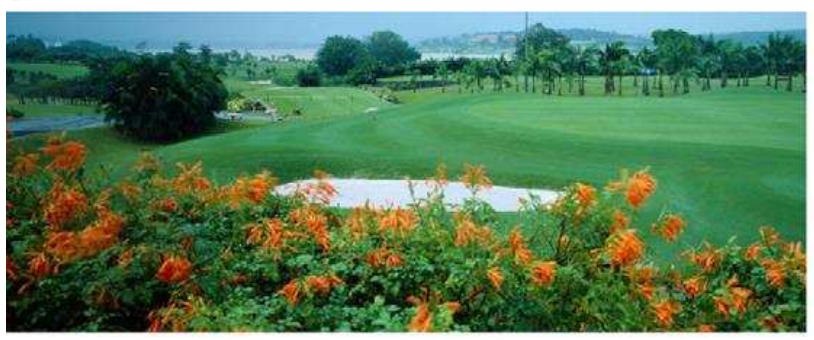

C

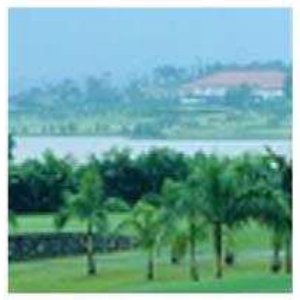

\section{d}
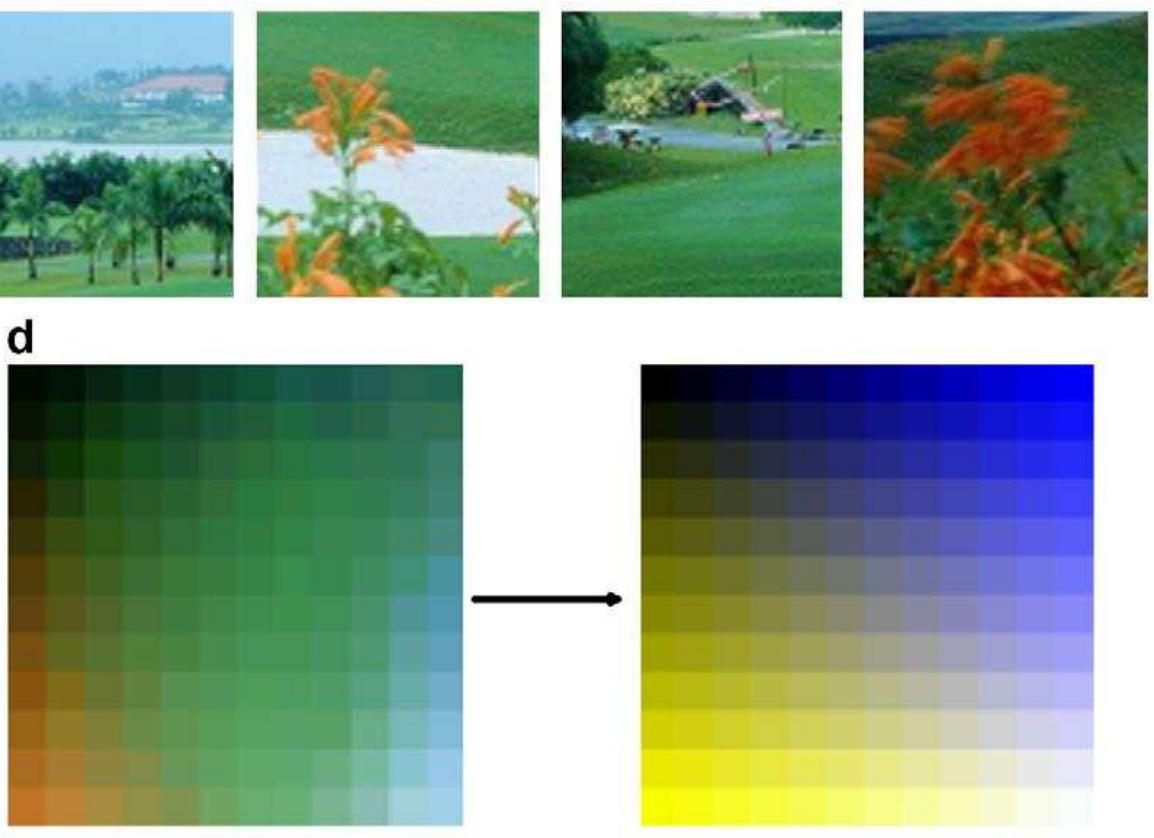

e

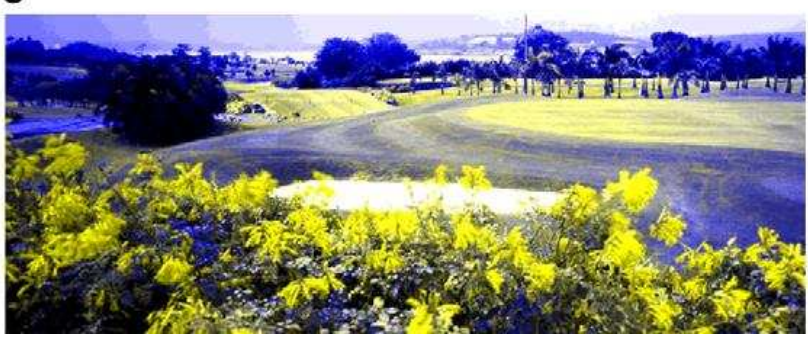

Figure 4.3: (a) The original image (b) The protanope simulation (c) Four selected sampled blocks as the input of the SOM (each 200 pixels by 200 pixels). (d) The original colors of "reference vectors" and their mapped colors (e)

(e) The final transformed image

This single image gives us a lot of insight about this method. In this method, the obvious color difference between red and green is changed to the difference between yellow and blue. It helps the protanope patient to distinguish between objects with different colors which usually look identical to them. At the same time it limits their color spectrum even more than before. As we can see in Figure 4.3(e), the whole scene is transformed into a bicolor image only consisting of yellow and blue. Of course it still serves the purpose but in some cases this method tends to limit 
the details that can be perceived. Suppose we have a large image composed of various colors. Narrowing down the color composition to only blue and yellow will result in limiting the details that user can perceive and possibly missing some information.

Another major disadvantage of this method is the human interaction factor. Although the authors claim that the SOM network needs to be trained only once when entering a new scene, the human interaction needed to choose sample blocks can be problematic. Wherever human interaction comes in action, the human error can be expected as well. We can expect that if wrong areas of the image are selected, the final result won't be appealing. Also there is always a chance that the user fails to select a part of the image that contains a unique color which consequently could result in a bad transformation once more.

One of the most important drawbacks of this method is its computational complexity which makes this method not suitable for real-time applications. For example, if we want to develop a web browser add-on or a mobile application to recolor images with one click to improve information perception for dichromatic patients, this method would definitely not fit the bill. Finally, Just like our method and many other color transformation methods, this method may also sometimes suffer from color discontinuity that does not exist in the original image.

On the bright side, this method could be beneficial when we want to recolor certain popular images like billboards, maps or books. In such cases the developer can pick the perfect samples and an ideal result will emerge. This method could definitely give better results than our method in large, complex images such as the one shown in Figure 4.3(a) but it does not pass the generality test and it is not a user friendly approach either. Finally, adaptability is a nice feature of this method. Unlike our method that has generality as its main focus, the self-organizing color transformation method uses different transformations for different scenes, rather than a fixed one.

Another very interesting image presented in [5] is illustrated in Figure 4.4(a). In the original image a green bull is embedded in the red background. As we can see in Figure 4.4(b), the protanope patients would perceive a deer from the original image. Figure 4.4(c) shows the selected blocks and 4.4(d) illustrates the final result of the self-organizing color transformation method. We show the result of our own color transformation method in Figure 4.4(e) and its protanope simulation in Figure 4.4(f). We can see that as claimed, the SOM method might give bolder result compared to our result. That being said, our result still helps the patient to perceive a bull in the image instead of previously seen deer.

Although this method might produce nicer looking images, it tends to over exaggerate certain features in the image. Authors claim that their method maintains the neighboring relations between colors. This statement is true in general but if we compare the original image in Figure 4.4(a) with their final result presented in Figure 4.4(d), we can see that for example in the 
highlighted area, green and dark brown dots are both transformed into dark blue. That might sometimes be the same case for our method as well but certainly not in this magnitude.

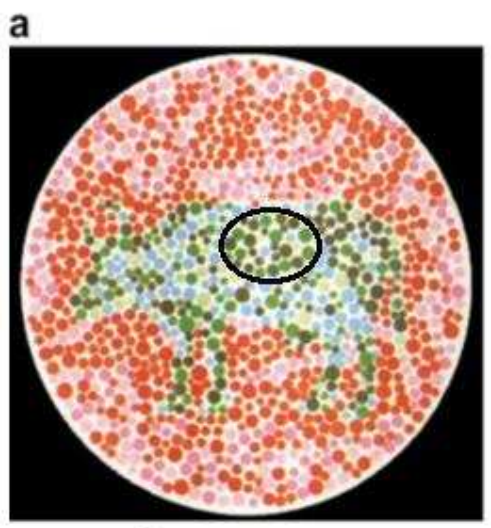

b

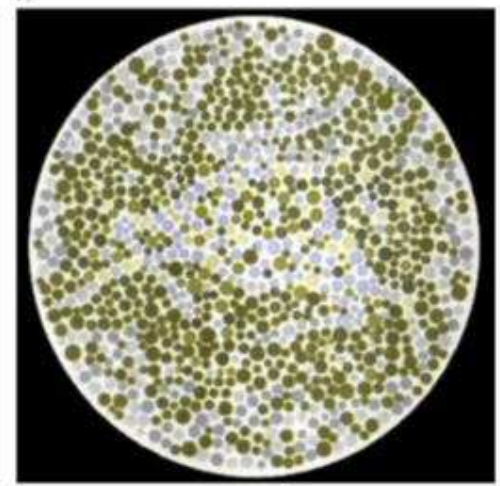

C

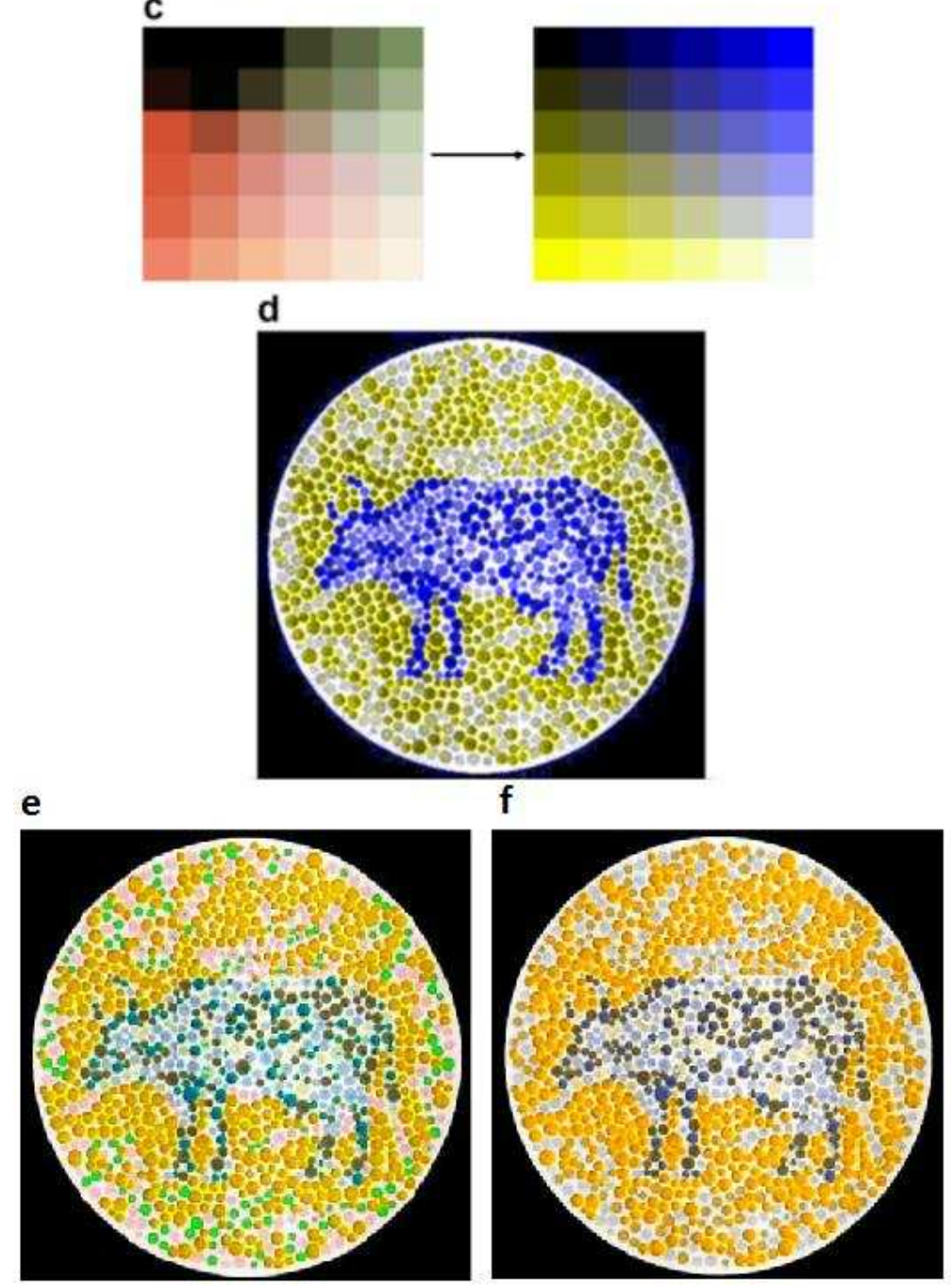

Figure 4.4: (a) The original image. (b) The protanope simulation of the original image (c) Reference vectors (d) The SOM transformed image (e) Our transformed image (f) The protanope simulation of our transformed image 


\subsection{Using an Interface}

In 2007 Jefferson and Harvey [9] presented a method to allow users to manipulate color composition of images under the control of a single variable. As we mentioned in Chapter 1, there are different types of CVD with various degrees of severity. The goal pursued in [9] is to modulate colors in the image so that when they are viewed by any CVD person, the perceived difference between any pair of colors is of the same magnitude as that perceived by a normal color viewer. The presented method permits color images to be modified interactively in a way that preserves color content for a particular class of color blind viewer.

The basic idea in Jefferson and Harvey's algorithm is to transfer the chromatic information of the defective cone across the two functioning cones in LMS space. For example, a protanope patient has no L cone. Therefore, he/she perceives no variation on the L axis; color stimuli with fixed M, fixed $\mathrm{S}$ and varying $\mathrm{L}$ collapse to the same color. Transferring this chromatic variation to the $\mathrm{M}$ and $\mathrm{S}$ cones allows the protanope to perceive the variation in color. The same fact is true in the case of deuteranopia and tritanopia for the $\mathrm{M}$ and $\mathrm{S}$ cones respectively.

In this algorithm the image data, specified as RGB tristimulus vectors CRGB, is converted to the LMS color space using CLMS $=$ TCRGB, where the elements of the matrix $\mathrm{T}$ are the LMS tristimulus values of the CRT primaries used in [13]. The CVD simulated color (C'LMS) is calculated from the original color (CLMS) using the projection rules also presented in [13]. Jefferson and Harvey [9] use an interface for their adaptation tool which is shown in Figure 4.5.

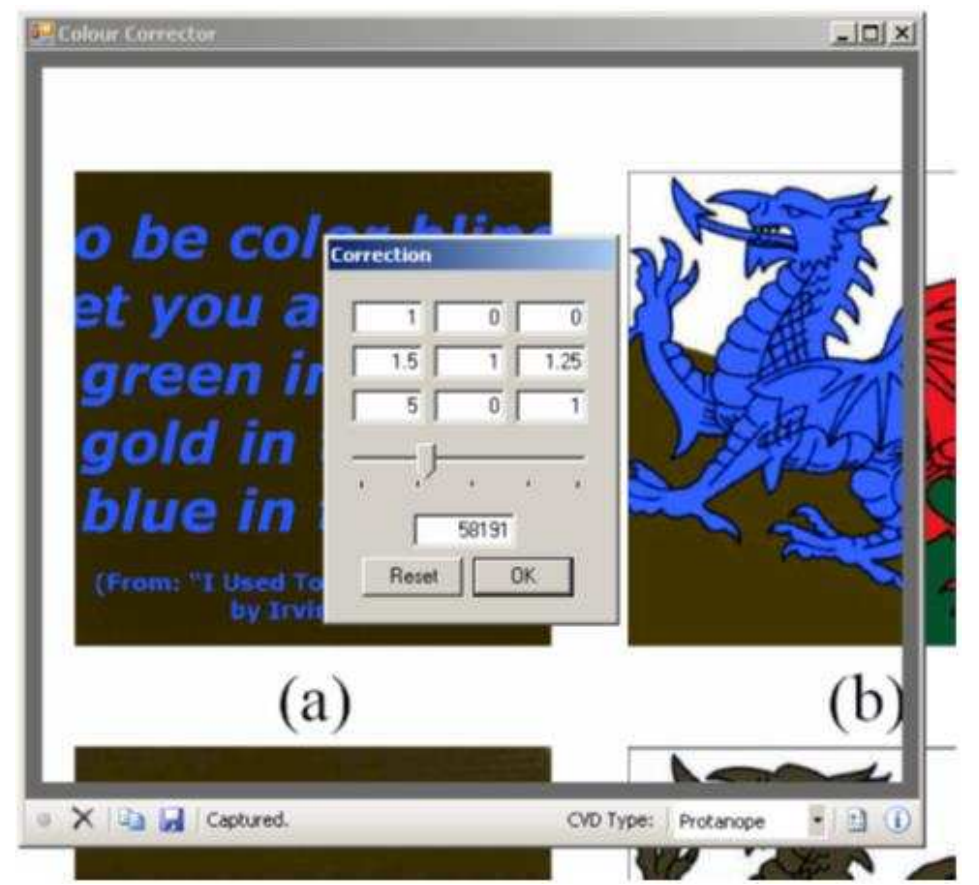

Figure 4.5: The interface for color adaptation tool presented in [9] 
This interface consists of a transparent window that can be layered over the desktop, allowing the user to recolor any region of the screen. On the bottom of this screen a small control panel has been implemented that allows users to capture, clear, copy or save the image. There is also an option to choose the type of color vision deficiency and also alternate between simulate and correction mode.

Here is how this interface works; the user places the transparent window over the part of the screen which contains the desired image and captures it. They need to specify their CVD type using the drop down menu in the control panel and then open the color correction control window by clicking the correction button. By moving the slider in this window, user can interactively recolor the captured image in real time. The parameters in this window vary between images and are dependent upon the color composition of the image and the type of CVD being corrected. Also, different users have different preferences and therefore select different parameters for the same image.

This is exactly a double-edge blade for this method. It can be nice that user can create the perfect color composition for their specific color vision but at the same time it gives room for error. The computation cost as well as the time it takes for one image to be processed might be more than our method but at the same time it gives users room to create their ideal color composition. We suppose this method could be most beneficial if the user wants to put time on fixing a specific image where computation cost is not an issue. However, as a general method we believe that our method gives results faster and easier for wider range of images without the need of external manipulation from user. Still we can count this method among the successful ones.

\subsection{Recoloring Images using Gray-scale Conversion}

As we mentioned before, observers with color deficient vision might perceive distinct colors to be indistinguishable and thus lose image details. So far we have presented our own method and two other methods for recoloring images to improve this detail perception. Another very interesting approach is introduced by Karl Rasche et al. [29, 31]. They present a method similar to what is used for converting color images into gray-scale in the case of recoloring images. However, there is a small problem which needs to be dealt with first.

During the conversion of a color image into gray-scale, colors that are pretty close in terms of luminance but have large differences in chrominance are mapped to similar shades of gray. Therefore, colors which were easily distinguishable in the original image, due to chrominance variation, might become indiscernible after a typical gray-scale conversion is performed. That is why Rasche et al. [29, 31] suggest a customized conversion for this specific task. 
They introduce an effective gray-scale conversion that tends to preserve the details in the original image by a perceptual match of relative color differences between the color and the gray-scale images. This means that the perceived color difference between any pair of colors in the original image should be proportional to their perceived gray difference. An example of this gray-scale conversion is shown in Figure 4.6. The left images show the original color image. In the middle we see a typical gray-scale conversion using the standard NTSC map to luminance. As we can see, for example in case of first row there is not much difference between red fruits and green leaves in the standard conversion as they have similar luminance and are mapped to roughly the same luminance in the resulting image. On the other hand, we can see that in the right image which is the customized conversion presented in [29, 31], fruits pop up and are far more distinguishable compared to the middle image.
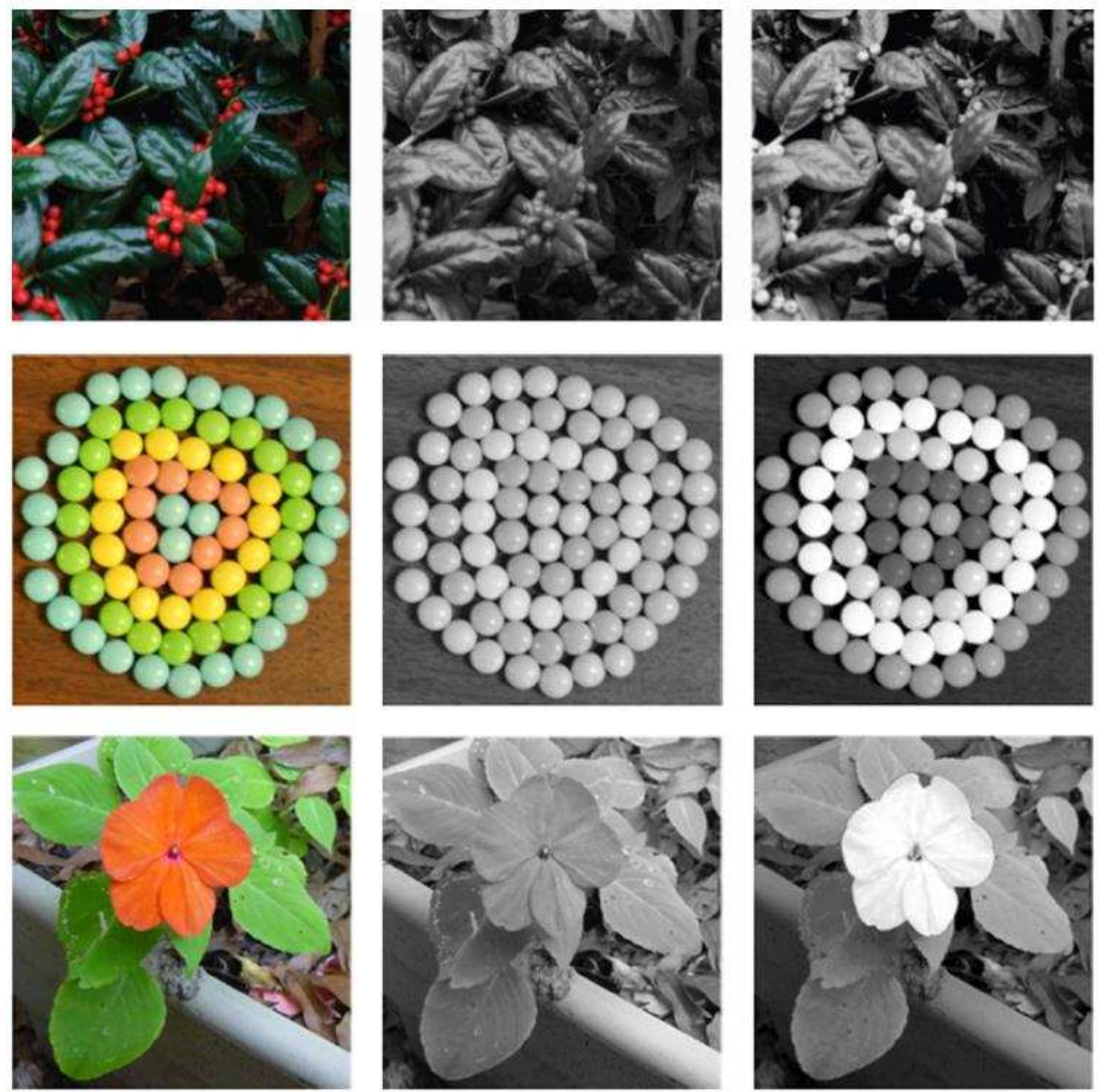

Figure 4.6: (left) Original color images (middle) A standard gray-scale conversion (right) A detail preserving grayscale conversion 
According to Rasche et al. [31], in the process of gray-scale conversion of images two conditions must be considered: First, contrasts within the image must be preserved. Colors that are already distinguishable in the original image should be represented by gray values that are also distinguishable. Second condition is to maintain luminance consistency. In a color image shadows, highlights and color gradients provide valuable information. An image which contains such cues needs to be dealt with carefully to prevent lightening shadows or darkening highlights.

The gray-scale conversion problem resembles the tone mapping of displaying high dynamic range images on a low dynamic range display as presented in [39]. In both cases, the objective is to preserve an abundance of visual information within the constraints of a limited gamut. The difference is that tone mapping is generally concerned with compression of the gamut range, whereas here the interest is in compression of gamut dimensionality.

This problem also resembles color gamut mapping for printing with a small set of inks. Usually in printing process the entire color gamut of an image might not be available. Therefore, the image must be mapped into the available gamut in a meaningful way. Stollnitz et al. [40] examine the problem of printing a color image with an arbitrary number of inks. For the case of a single ink, their solution is also to use luminance, i.e., the standard mapping. Rasche et al. [29] took advantage of this fact to present their recoloring method. They attempt to reproduce colors using only one or two basic colors. In this particular problem, colors blue and yellow have been chosen to represent the image as they are counted among safe colors for dichromats.

Figure 4.7 illustrates the result of their presented method and also comparison of their results with ours. On the top left of this image we have the original picture. Right below it we have the protanope simulation of that picture. In the middle the final result of the presented method in [29] is shown. As we can see the whole picture is transformed and recolored using only yellow and blue colors. We added our own results to the right side of this image. On the top right we have the result of our color transformation and on the bottom right we see how it is perceived by a protanope user.

The difference in this specific example is not that significant as there are two dominant colors in the original image as well. However, if we take a more complex example the final result from this method will be similar to what we saw in Figure 4.3 as this method will recolor images into a bicolor image as well. The authors claim that their method is fast to compute and easy to implement as opposed to some other similar procedures. Another advantage is that this linear method does not require tuning, in the form of a neighborhood-size parameter, which frequently appears in nonlinear methods. 

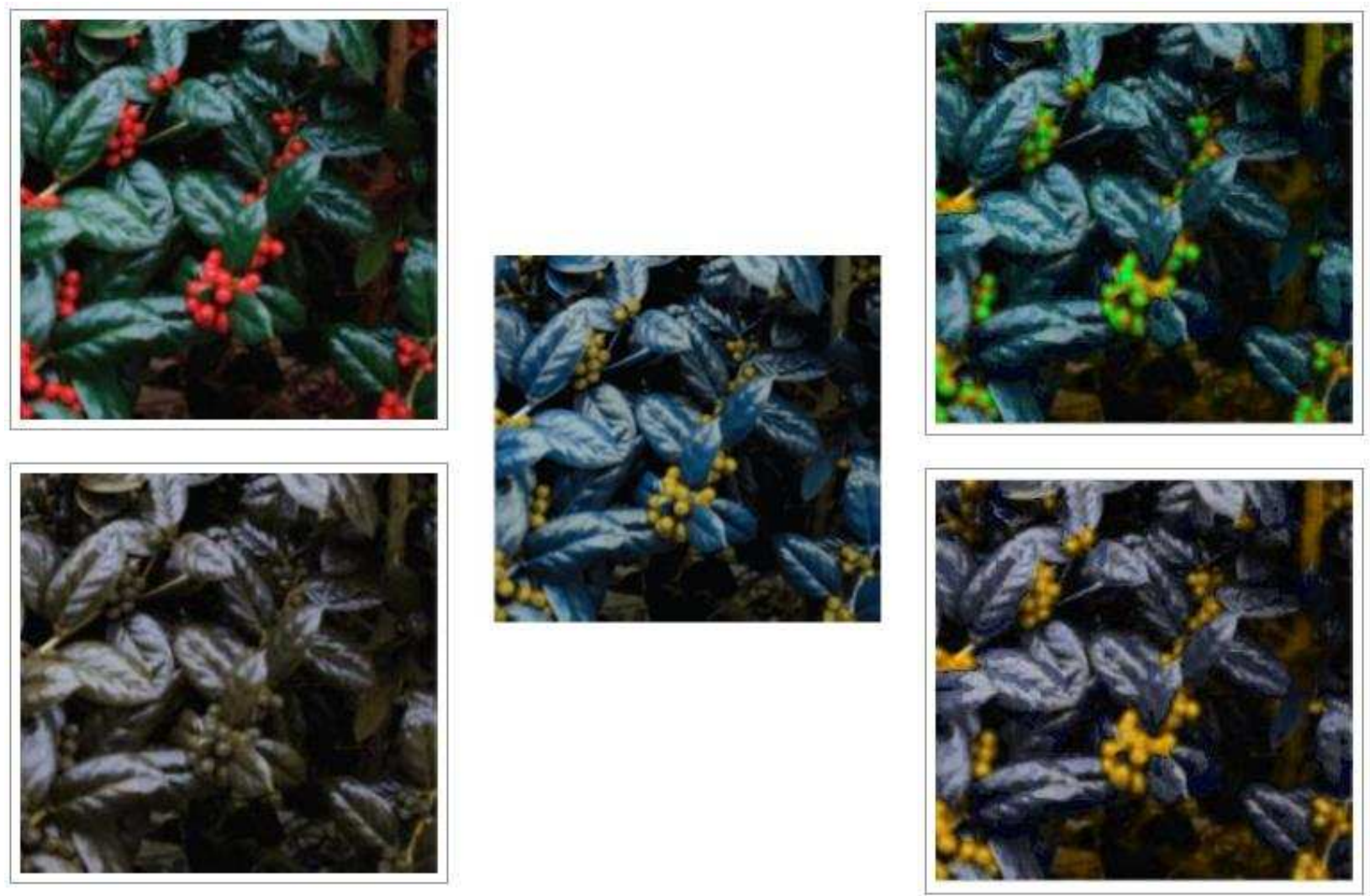

Figure 4.7: (top left) The original image (bottom left) The protanope simulation of the original image (middle) The transformed image after the process presented in [13] (top right) The recolored image after our color transformation method

On the negative side, just like many other recoloring approaches, this method sometimes produces results of questionable value. For instance, as authors state it themselves, the linear gray-scale transform also breaks down when the image contains large patches of black and white [13]. We can also see that similar to our results, sometimes we will observe unnatural colors such as blue leaves in this picture! Another limitation to this method is the fact that it has difficulties with images that exhibit smooth variation along multiple color dimensions, and it does not offer any constraints on luminance consistency [31].

Overall this method can be considered a successful method as well. However, we believe for the sheer purpose of increasing the amount of perceived information and in terms of general usage, our method works better. Suppose we apply this approach on the image of different fruits presented in the Figure 3.16; in the resulting picture after applying the recoloring process explained in this section, the color deficient user will most probably have even a harder job to distinguish different colors of the same fruits as the whole picture is recolored only with blue and yellow. Again, we believe that this method could prove to be valuable in cases where we need a 
specific image to be transformed but when it comes to global usage for wide variety of images, it might come short.

\subsection{Information Preserving Color Transformation}

All of the methods introduced so far, including our own method, are mostly concerned about how the final image is viewed by a color deficient user. The main focus in all of these methods is to generate images that are more comprehensible to color deficient users and the fact that the original image may sometimes look very unnatural to viewers with normal vision is of no importance to the majority of these methods.

In the real world, images in public places may be simultaneously observed by people with normal vision and those who have CVD. For example, in a public transportation system, many advertisements and traffic maps are delivered in colors. Without any consideration for color deficient users, they might have difficulty finding their destination using those images. On the other hand, if the sole focus is helping CVD users, the recolored images may look annoying to thousands more people with normal color vision. Huang et al. [30] present a color transformation method that aims to preserve the color information in the original images while maintaining the recolored images as natural as possible.

In order to keep the recolored image as natural as possible, Huang et al. [30] introduce three rules. First, the recolored image has the same luminance as the original image. Second, colors with the same hue in the original image still have the same hue after recoloring. Finally, the saturation of the original colors is not altered after recoloring.

Moreover, two criteria are introduced in this method, one for detail preserving and the other for preserving naturalness. For each color pair in the original domain, first the perceived color difference is calculated with respect to a person with normal vision. Then, for the corresponding color pair in the transformed color domain, the perceived color difference with respect to a person with protanopia is calculated. The key is to keep these two perceived color differences as similar as possible. Hence, an error function is defined as follows:

$$
E_{\text {detail }}=\sum_{i} \sum_{j}\left(\left|C_{i}-C_{j}\right|-\left|\operatorname{SIM}\left(T\left(C_{i}\right)\right)-\operatorname{SIM}\left(T\left(C_{j}\right)\right)\right|\right)^{2}
$$

In this formula, $\mathrm{i}$ and $\mathrm{j}$ range over the colors contained in the images, $|$.$| is a perceptual color$ difference metric, $\mathrm{T}($.$) is the recoloring function, and \operatorname{SIM}($.$) denotes the simulated color$ perception using Brettle's algorithm. Color details of the original image can be preserved by minimizing this error function. 
On the other hand, this attempt to preserve the details of the original image must be monitored carefully as dramatically modifying the color perception of the colored image might make the recolored image extremely unnatural for normal viewers. For this reason, another error function is defined as follows:

$$
E_{\text {naturalness }}=\sum_{i}\left(\left\|C_{i}-T\left(C_{i}\right)\right\|\right)^{2}
$$

In this case, i ranges over all the colors in the original image. Minimizing this error function shortens the color distance between the original colors and the corresponding remapped colors. To preserve both details and naturalness, these two error functions are combined together to form an objective function using the Lagrange multiplier with a user-specified parameter.

$$
E_{\text {total }}=\overline{E_{\text {detail }}}+\lambda \overline{E_{\text {naturalness }}} \text {. }
$$

This objective function is then minimized to obtain the optimal settings. By choosing different values of $\lambda$, users may adjust the tradeoff between details and naturalness. A larger $\lambda$ makes the recolored image more natural for normal viewers, while a smaller $\lambda$ makes the recolored image more comprehensible for color-deficient viewers.

Figure 4.8 illustrates an example presented in [30]. The color contrast between red flower and green leaves is lost for people with deuteranopic deficiency. Figure 4.8 (b) shows the recolored image resulting from Rasche's method from Section 4.3 and figure (g) is its deuteranopic simulation. We can see that although Rasche's method may create great contrast for deuteranopic people, the naturalness of the recolored image is extremely poor for people with normal vision. The same can be said about the result of our recolored image shown in Figure 4.8 (c). As we can see in Figure (h), the recolored image can be perceived with great contrast for our color deficient user, but we have a yellow flower with blue leaves which is far from the natural color of the original image.

The last two images on the right show the effect of the parameter $\lambda$ in this recent method. Figure (d) is the result of applying $\lambda=0.1$ in the objective function. The recolored image can be perceived with natural and acceptable colors for a normal viewer and the simulation in Figure (i) shows that a deuteranope can see a reasonable contrast between flower and leaves. On the other hand we can see the effect of removing naturalness from the equation in Figure (e). In this image the parameter $\lambda$ has been set to zero completely ignoring the naturalness. We can see that the resulting recolored image has orange leaves instead which is a bad result in terms of naturalness. 


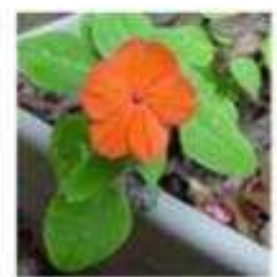

(a)

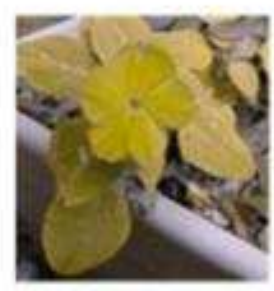

(f)

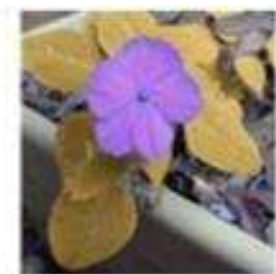

(b)

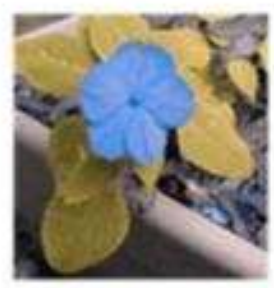

(g)

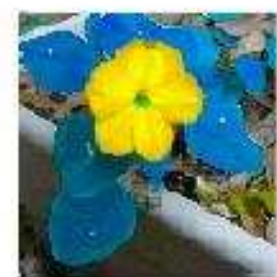

(c)

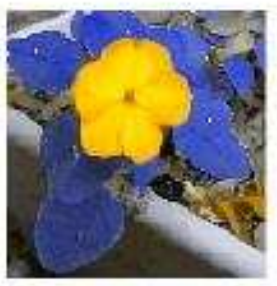

(h)

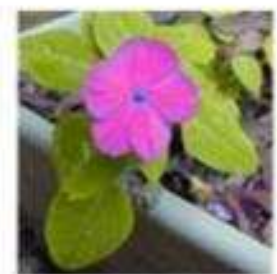

(d)

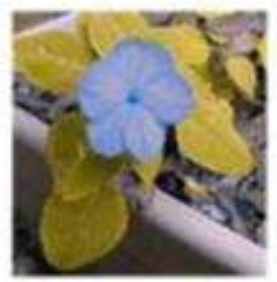

(i)

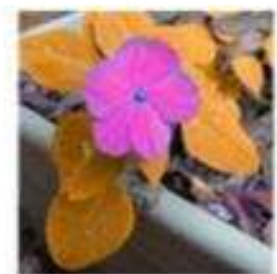

(e)

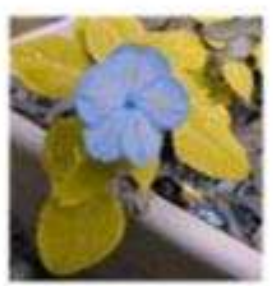

(i)

Figure 4.8: (a) The original image (b) Recolored image by Rasche's method (c) Recolored image by our method (d) Recolored image by information preserving method with $\lambda=0.1$. (e) Recolored image by information preserving method with $\lambda=0$ (f-j) The deuteranopic simulation of each image on the top row

\subsection{Summary}

In this chapter we tried to introduce some alternative methods trying to solve similar problems. We presented four distinct methods each of which emphasizing on other aspects of recoloring images. In each section we gave a short introduction on how authors tried to solve the problem and which aspects were the most important ones for them. Wherever we could, we tried to compare our own method and results with what other researchers brought to the table. We can use the concepts presented in this chapter as building blocks of a more general method which benefits from advantages of each of these methods and preventing the drawbacks of each one of them. This method, however, requires a lot more elaboration which is out of the scope of a master's thesis. 


\section{Chapter 5}

\section{Analysis of Results \& Conclusion}

As we mentioned briefly at the beginning of Chapter 3, we plan to validate our method both objectively and subjectively. In Chapters 3 and 4, we tried to see if our method, or alternative methods for that matter, can be beneficial for color vision deficient users through simulation of their vision before and after color transformation. Simulation of such kind is an acceptable and popular approach in most cases as most of the researcher might not be color blind themselves. Hence, some sort of simulation tool is needed to distinguish the changes and evaluate whether those changes are headed in the right direction or not.

First portion of this chapter is dedicated to analyzing the results of our own survey on actual color blind users. Although we could prove the effectiveness of our method through simulation tools such as the one which we explained in Chapter 2, we were motivated to validate our approach by putting it to test in the real world as well. That is why we asked a group of users with color vision deficiencies to fill out a questionnaire consisting of various test images before and after performing our color transformation method.

The second part of this chapter integrates everything we talked about in this thesis and comes to a conclusion as well as opening a path for the future work on this project. In order to reach a more generalized method that can work not only for the dichromats but also for other types of color vision deficiencies, we came up with the idea of combining our method with the personalized simulation method presented by D. R. Flatla and C. Gutwin [2] to reach a personalized color transformation method. This however, is far beyond the scope of this thesis and needs further work in the future to come to reality. 


\subsection{Experimental Results}

Although we proved the effectiveness of our proposed algorithm through the existing simulation tools, we wanted to get a feedback from users who are actually dealing with CVD. For this reason, we prepared an online questionnaire with different questions that try to figure out if the transformed images work better for CVD users or not. Over 250 entries from users from all over the world were submitted.

Since many users wanted to see how the test looked like, even if they were not color blind themselves, a lot of unfinished answers were included. We also wanted to prevent non-CVD users to submit valid entries so we devised a few trick questions that tend to spot normal users from actual color blinds. Finally, based on the answers we had to remove all the users who were blue-yellow color blind. This step was done because our method is optimized to work with redgreen colorblindness and it might have negative effect on users with blue-yellow colorblindness.

In this section, we will analyze the answers and quantitatively prove that our method actually improves the experience dichromatic users can have from a digital image. After applying all filters described in the last paragraph, we ended up with 39 valid entries.

The first point worth mentioning is the fact that the majority of color blinds don't know the type of their condition. Figure 5.2 shows the distribution of different types of colorblindness among our valid submissions. We can see that $72 \%$ of participant did not know the type of their color vision deficiency. There have also been a few requests from some participants asking us to detect the type of their colorblindness based on the answers they provided which we obliged.

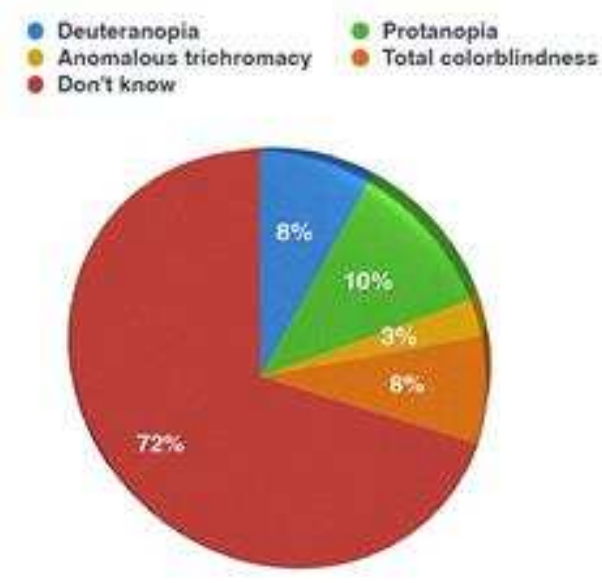

Figure 5.2: The distribution of different types of CVD among our participants. 
In the first question we asked participants to decide which of the two images presented in Figure 5.3 gives them more details. We ask them to choose the picture in which they can see more details on the dragon. Of course, none of the participants knew which image is the original image and which one is transformed by our method. The interesting result was that all 39 participants picked the transformed image over the original image. This is refreshing as our method proves to be working even for cases like total color blindness or anomalous trichromacy for which our method was not optimized.
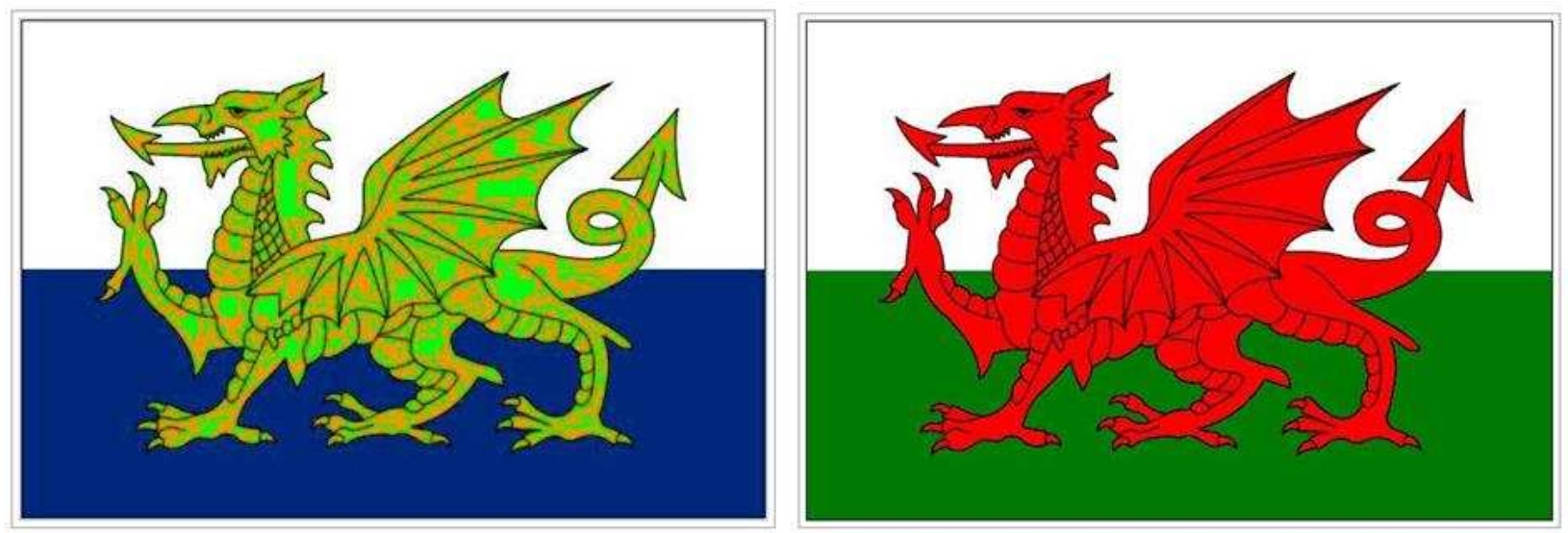

Figure 5.3: (left) Transformed image (right) Original image

In the next two questions we asked the participants which number they see on Figure 5.4(a) and which number is perceived from Figure 5.4(b). The original image was presented first and our transformed image followed it in the next question. The simulation step from chapter 3 showed that users with protanopia should perceive number ' 2 ' from the original image. However, as Figure 5.2 illustrated our participants were not limited to protanopia. Figure 5.4(c) shows the distribution of numbers that users saw from the original image and Figure 5.4(e) illustrates it on a graph.

Our goal is to see if our method can improve the perception of users from these images so we discarded the entries of users who saw number ' 42 ' from the original image and tried to see if our transformed image helps those users who got it wrong, find the right answer or not. Among the 30 users who answered 2, 4, 47 or even could not see anything from the original image, 29 of them could recognize the number ' 42 ' from the transformed image. The remaining one participant, who originally could not see anything, had the improved answer of ' 4 '. We can see this distribution in Figure 5.4(d) and (f). We also put the two images in Figure 5.4(a) and (b) together in another question and asked them which of the two images is perceived closer to the right answer which is ' 42 ' and all the users who originally got the wrong answer, except one, chose our transformed image over the original image. 

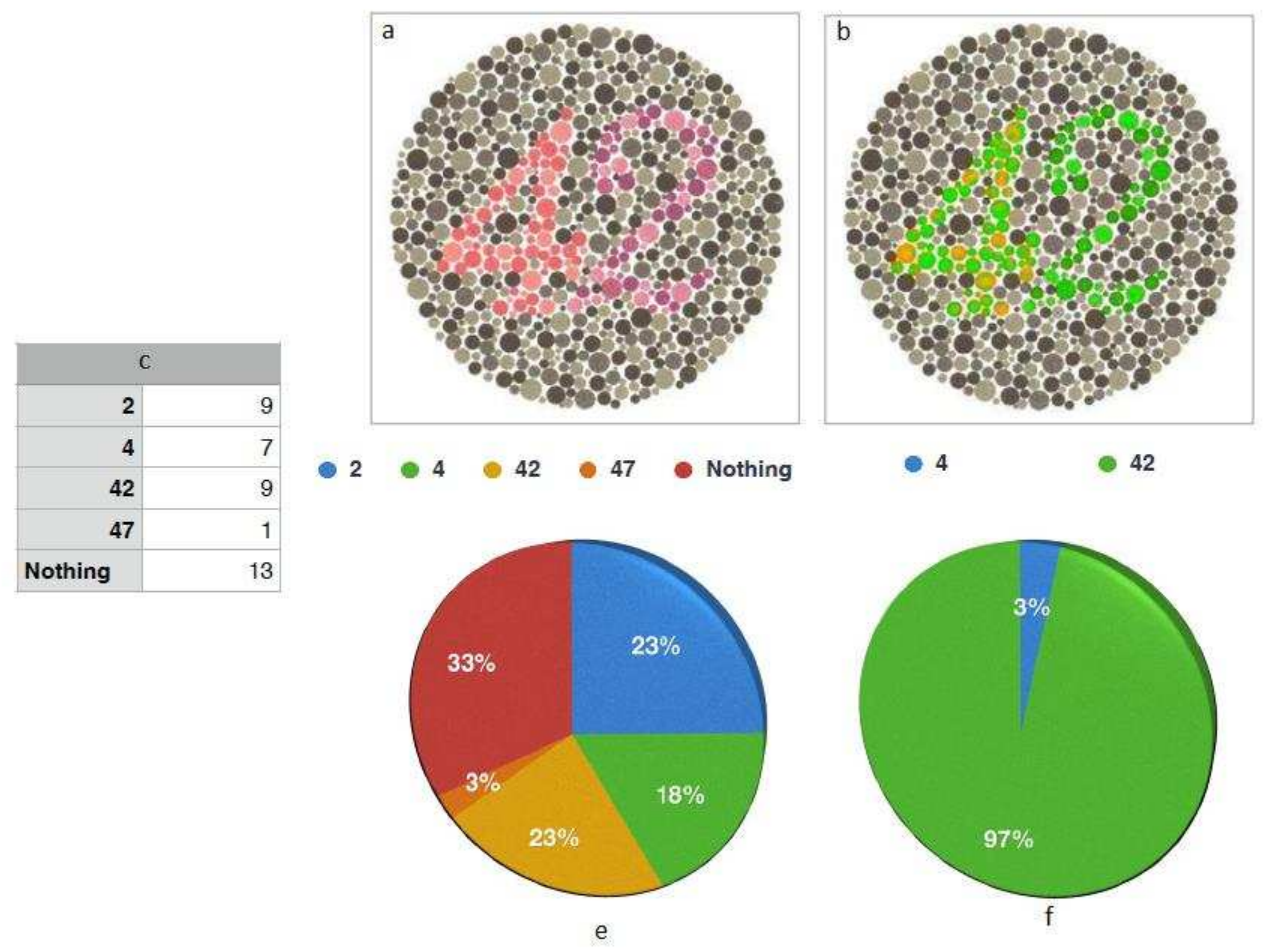

\begin{tabular}{|r|r|}
\hline \multicolumn{1}{|c|}{ d } \\
\hline 4 & 1 \\
\hline 42 & 29 \\
\hline
\end{tabular}

Figure 5.4: (a) Original image (b) Transformed image (c) Distribution of answers to the original test image (d) distribution of answers of people who originally did not see '42' (e),(f) Graphs corresponding to tables 'c' and 'd '

In another question we asked the participants to choose the image in which they can see number ' 8 ' more clearly, see Figure 5.5. One image was the original image and the other was the result of it passing through our color transformation algorithm. All 39 participants chose our result over the original image which proves that our method is very effective in color compositions like this. Figure 5.5 shows the images presented in this question.
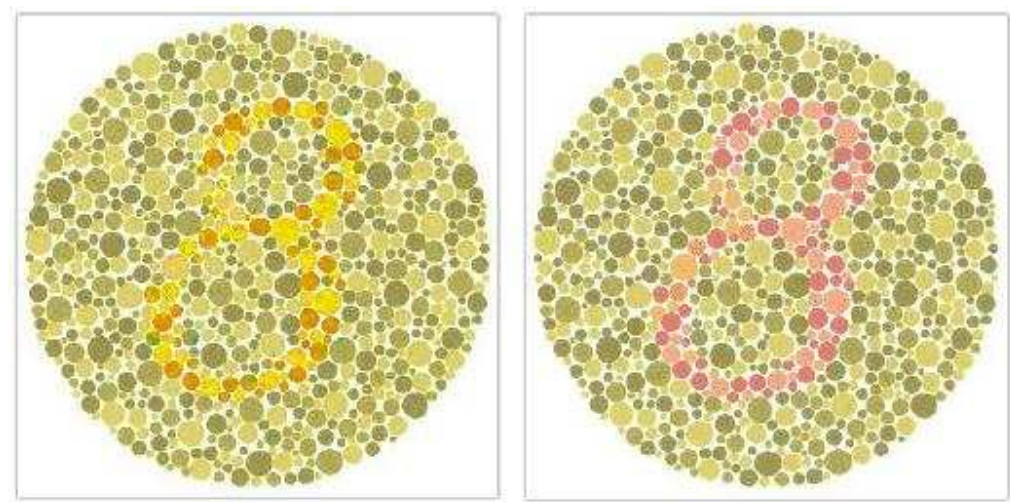

Figure 5.5: (left) Transformed image (right) Original image 
In a similar question to what we showed in Figure 5.4 we tested a totally different color composition. Again in the first question we asked about the number they could see in the original image and in the following question we asked them the same concept, this time on the transformed image, see Figure 5.6. The results to the original image showed that this image was very challenging for the majority of users and only two of them could initially figure out the number ' 5 ' among the dots. However, after asking them the same question about the transformed image all 39 participants got it right this time.
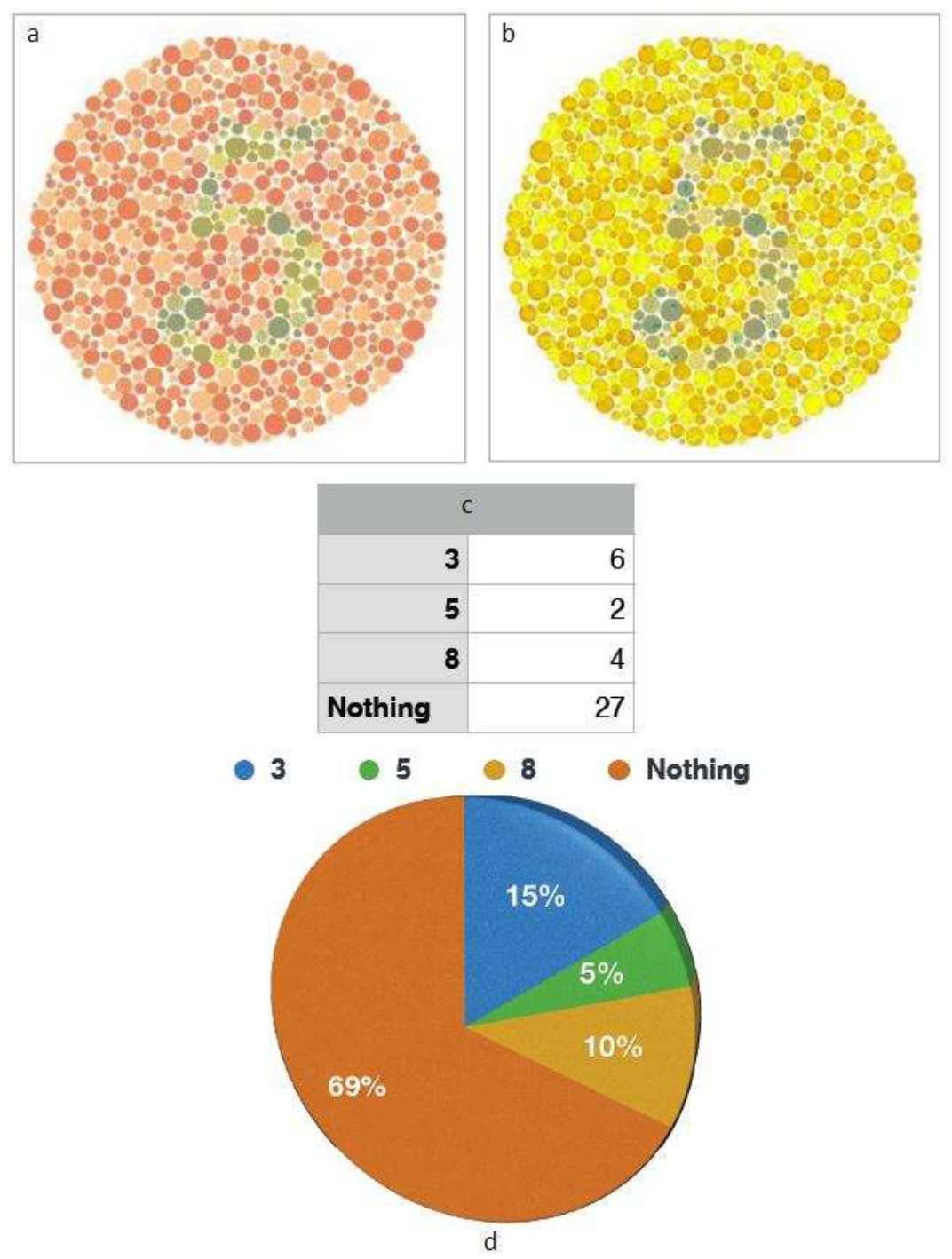

Figure 5.6: (a) Original image (b) Transformed image (c) Distribution of answers to the original test image (d) The graph corresponding to table ' $c$ '. 
Another similar test image was the one presented in Figure 5.7(a). This image proved to be very challenging for most of the participants and split answers were given to this question. Figure 5.7(a) shows the original test image and 5.7(b) is the transformed image. The table and graph in 5.7(c) are the distribution of answers based on the original image. Figure 5.7(d) is related to the results given to the transformed image. In Figure 5.7(e) we investigated the distribution of answers of those participants who did not originally pick number ' 74 ' as the answer.

We can see that in the original image only $8 \%$ of the participants could find the right answer. However, among the people who got the wrong answer, 39\% got it right after the transformation. We can also observe that only 2 participants still saw nothing in this picture. Even though the percentage of people who gave the exact right answer after transformation is only $39 \%$, the rest of the answers such as 21, 24 and 71 are also improvement to the original answer considering the fact that this particular test image is among the hardest ones to recognize. In fact even the simulation shows that it could be challenging to get it right.

On a negative side, 3 of the participants who could originally find the right answer (since they were most probably colorblind of the type yellow-blue), chose different answers on the transformed image which was totally predictable. Our method is optimized around fixing the recognition problems of people with red-green colorblindness and if a person with yellow-blue colorblindness views our transformed image it might have negative effect.

Finally, we decided to investigate the effect of our algorithm on the answers of people who could see nothing from the original image. Figure 5.7(f) shows the distribution of those 19 individuals. From this group $47 \%$ got the acceptable answer of ' 71 ', $32 \%$ found the right answer ' 74 ', $11 \%$ gave the answer ' 21 ' and only two participants could still see nothing after transformation. All and all, we can say that even in this extreme case, our method comes out strong and proves to be useful. 

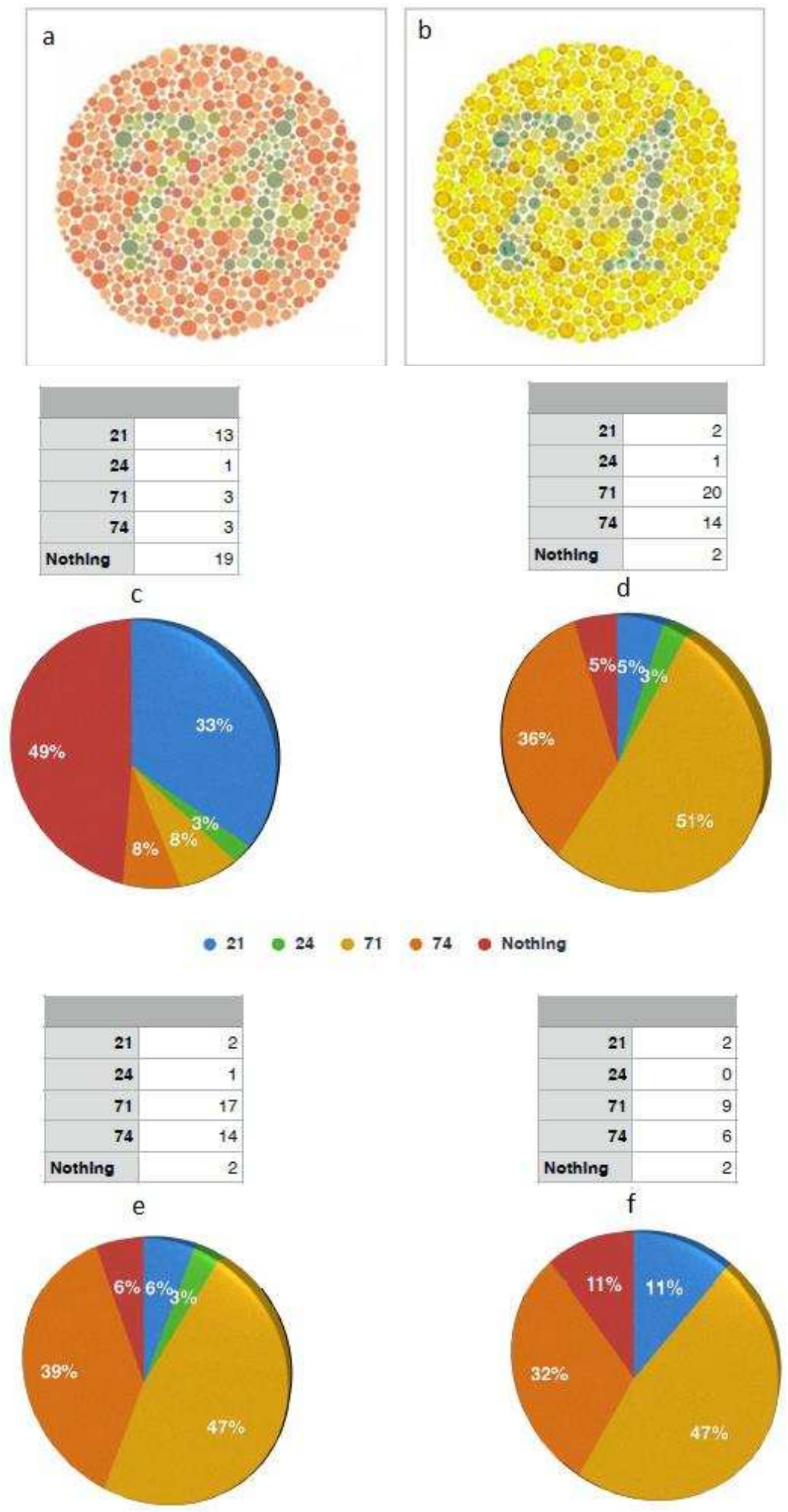

Figure 5.7: (a) Original image (b) Transformed image (c) Distribution of answers to the original image (d) Distribution of answers to the transformed image (e) Distribution of answers of those who originally did not pick '74' as their answer (f) Distribution of answers of those who could originally see nothing from the test image. 
In another question similar to what we saw in Figure 5.5 we asked the participants to tell us in which one the two images of Figure 5.8(top) they can see number 6 more clearly. Again, all 39 participants unanimously chose our transformed image over the original test image. In the final questions of this questionnaire we gave the original image of the fruits presented in Chapter 3 and the transformed version of it and asked the participants which one of these two images they would prefer, see Figure 5.8(middle).

More precisely, we asked them to choose the image in which they can see more details such as detecting different types of peers or grapes. Twenty three of the participants believed that they would choose our result in spite of having discontinuity in colors in parts of the image. In the end we put the confusing image presented in Figure 5.8(bottom) to detect who is actually colorblind and who pretends to be. Everyone, who did not see a "deer" in the original image, could either have normal color vision or yellow-blue colorblindness and in both cases they could not provide valid answers to our results. Therefore, their answers were disqualified.
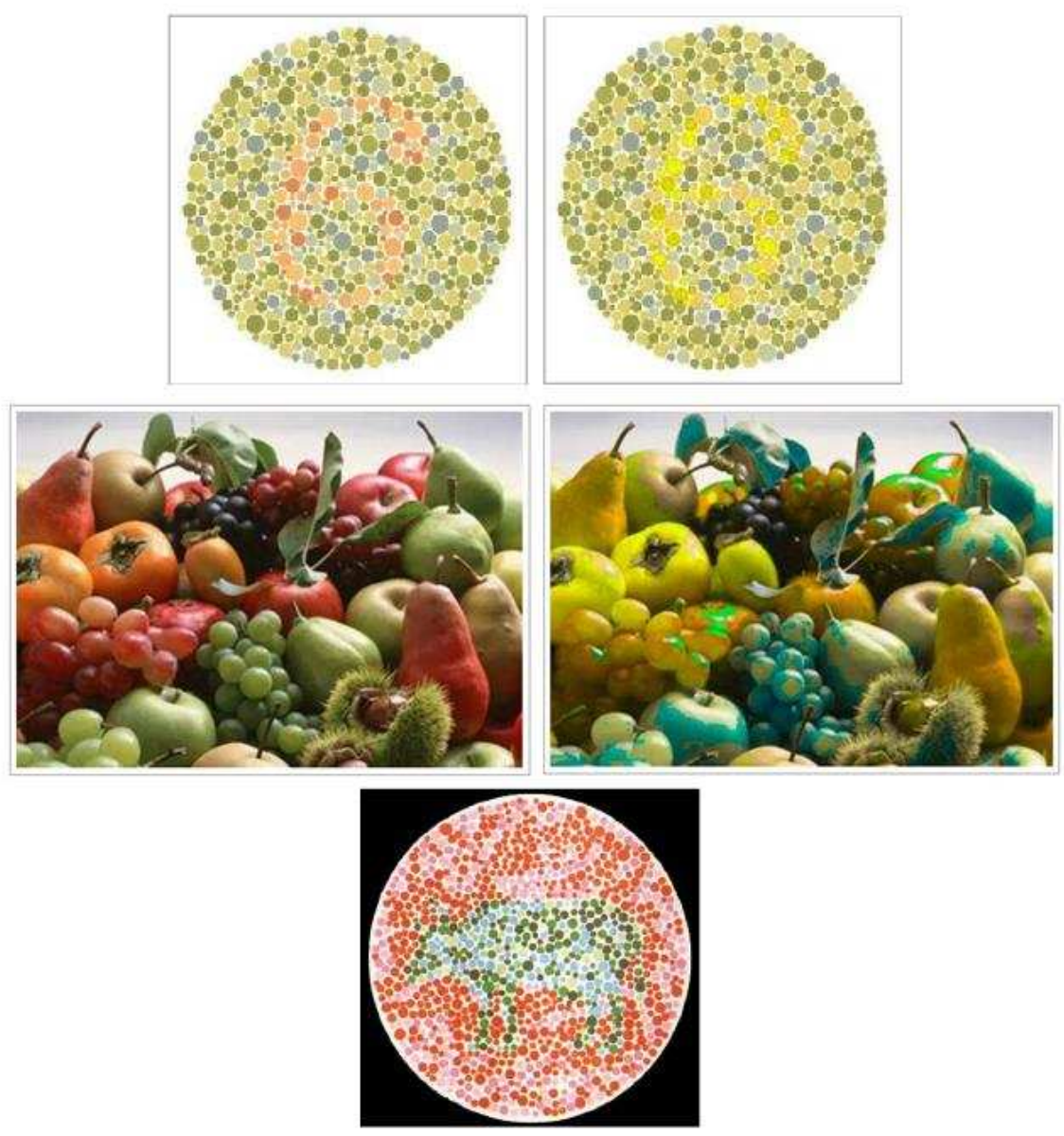

Figure 5.8: (top and middle) Comparing the original image on the left with the transformed version on the right. (bottom) The confusing image of the deer which can spot color blinds from the people with normal color vision 
In the final portion of the questionnaire, the participants were given the opportunity to comment on the project. Many of the participants cheered us up and wished us good luck on the project. Here are a few of those comments:

- This seems very interesting and is actually really helpful seeing as most of the original images I could barely recognize the shape in and then in the altered ones, the images were extremely clear (United Kingdom - Deuteranopia)

- The yellow things provide much more contrast than the usual blue adjustments for colourblind, it works really well! (Netherlands - Type unknown)

- Glad to see people taking an interest in this issue that I think is largely ignored (United Kingdom - Type unknown)

- Actually I have no idea which kind of colorblindness I have, could you tell me? (Germany - Type unknown)

\subsection{Future work}

A simulation tool similar to what we explained in Chapter 2 can serve our purpose in the case of dichromatic vision, but it certainly cannot simulate all different types of color vision deficiencies. In fact most of the approaches focus on simulating the dichromatic vision as it is a straightforward problem and does not require extra information such as degree of severity which is needed for simulating anomalous trichromacy.

An interesting approach presented by D. R. Flatla and C. Gutwin [2] tries to simulate a more exact representation of what a particular person actually sees. This approach is based on a specific empirical model of the actual color perception abilities of a person with CVD. This is actually relevant to this thesis because it opens doors for our future work on improving our method to be compatible with more types of CVD.

First let us have a look at why a personalized simulation of color vision would be beneficial over the typical simulation tools such as what described in section 5.1.1. We already mentioned the first shortcoming of the existing tools. They are mostly simulating dichromatic vision which is a severe type of CVD. However, $75 \%$ of the cases of genetically-caused CVD involve a less 
severe type of CVD (anomalous trichromacy) [2]. This results in dichromatic simulations being too extreme in most cases of CVD.

The second problem is that even if a simulation tool fixes the first problem and simulates more types of CVD, it still needs to know the specific type of CVD the user has. This is problematic because most users, as we also noticed in our experimental results, do not know the type of their condition. Moreover, the existing tools only simulate one type of CVD at a time, but there are cases where an individual has a combination of genetic and acquired CVD at the same time. The method presented in [2] addresses all these problems and creates a model that exactly captures the type and severity of the person's color vision deficiency. This could include cases such as anomalous trichromacy or multiple simultaneous deficiencies.

The process of personalized simulation of color vision introduced by D. R. Flatla and C. Gutwin [2] starts by determining the type and severity of the user's CVD. This is done by utilizing empirical measurements of user's color differentiation ability. Once the type and severity is recognized, a two-stage reduced-severity dichromatic simulation is employed to simulate the user's color vision.

Another interesting note pointed out in [2] is the fact that most common dichromatic simulation tools assume that everyone without CVD possesses identical color perception abilities, but researches show that there are variations in color perception abilities among the non-CVD population [41]. Therefore, D. R. Flatla and C. Gutwin [2] consider the color vision abilities of both the CVD individual and non-CVD viewer during their simulation.

Once the primary and secondary CVDs are identified, the amount of shift along each confusion line needs to be determined. Due to the impossibility of protanopia and deuteranopia cooccurring, this step can be simplified to identifying a "red-green" shift amount and a "blueyellow" shift amount. To find the primary CVD shift amount, the absolute difference between the major axis half-length and the average of the major and minor axes' half-lengths for the nonCVD ellipse is calculated.

This personalized simulation is claimed to be more accurate than the typical color vision simulation tools. Moreover, pairs of participants (one with CVD and another with normal vision) have been asked to discuss what they see in images of everyday scenes and they found new insights into the details hidden in the image when they looked through the personalized simulation. Now, what we can do in future is to integrate this personalized simulation method with our own method to generate a personalized color transformation method that can work with different cases of color vision deficiencies. We can also work our way towards a real-time recoloring algorithm to change the colors of the image or video sequence on the fly. Any of these ideas coming to life could potentially enhance the life of millions of people all around the world. 


\subsection{Conclusion}

We introduced a new method to help the CVD users, figure out the differences between colors within an image. In the field of color transformation, there have been quite a few different approaches to tackle the same problem. While the ultimate goal for most of these approaches is to improve the perception and eliminate the confusion, the way to reach this goal and the priorities are what separates these methods. In our case, we decided to recolor the image by taking advantage of the great facilities that HSV color model give us. Since this model decouples the color information from the grayscale information, we could simply keep the intensity constant and manipulate the hue and saturation of those colors that tend to generate confusion for our users.

The most important part of our method is to recognize the safe and troublesome colors. We found these colors by simulating the protanopic vision of a color card that we designed ourselves and comparing the distance between colors in the original color card with the colors in the simulated color card. The colors whose hues are perceived almost identically are counted as safe colors while colors whose hues are perceived dramatically different should be treated as troublesome colors. The actual hue and saturation manipulation is performed on those colors based on some pre-defined rules.

The important fact to mention here is our priority. Our main focus was on helping the color blinds see the details in the image better than before and if that meant some parts of some images might end up looking weird or unnatural to a person with a normal color vision, so be it. We tried our best to exploit the limited color spectrum that people with this kind of condition possess and emphasize on the distance between colors rather than identification of the original colors.

In order to validate our work, we took use of an online simulation tool. For each image, we simulated the way that it is seen by a dichromat originally and the way that the transformed image would be perceived. Then we judged ourselves if the perception has improved or not because of the changes that our program apply on the image. We also got feedback from 39 actual color blinds by asking them to fill in an online questionnaire. Overall, we can say that our method certainly fulfill the objectives that we had in mind and come out as a successful method which can help the color blinds a great deal. This method has a lot of room to grow. It is highly customizable and can be mixed with other methods to be able to help even larger groups of color blinds. 


\section{References}

[1] "Color Blind Essentials", Free eBook n Colblindor.com.

[2] D. R. Flatla and C. Gutwin. 2012. "So that's what you see: building understanding with personalized simulations of colour vision deficiency". In Proceedings of the 14th international ACM SIGACCESS conference on Computers and accessibility (ASSETS '12). ACM, New York, NY, USA.

[3] R. C. Gonzalez and R. E. Woods. 2008. "Digital Image Processing", Third Edition. Prentice Hall.

[4] H. PR. Albuquerque, "Color Vision Deficiency Tutorial”, By Richmond Products.

[5] Y. Ma., X. Gu., and Y. Wang. 2009. "Color Discrimination Enhancement for Dichromats Using Self-organizing Color Transformation", Information Science, Vol. 179, pp. 830-843.

[6] G. Iaccarino., D. Malandrino., M. D. Percio., and V. Scarano. 2006. "Efficient EdgeServices for Colorblind Users", In Proceedings of the 15th international conference on World Wide Web (WWW '06). ACM, New York, NY, USA.

[7] J. Nam., Y. M. Ro., Y. Huh., and M. Kim. 2005. "Visual content adaptation according to user perception characteristics". In Trans. Multi. 7, 3 (June 2005), 435-445.

[8] J. Birch. 2001. "Diagnosis of Defective Colour Vision", $2^{\text {nd }}$ editon. Oxford University Press.

[9] L. Jefferson and R. Harvey. 2007. "An interface to support color blind computer users". In Proceedings of the SIGCHI Conference on Human Factors in Computing Systems (CHI '07). ACM, New York, NY, USA. 
[10] Y. K. Kim., K. W. Kim., and X. Yang. 2007. "Real Time Traffic Light Recognition System for Color Vision Deficiencies”, In Proceedings of IEEE Xplore, pp. 76-81.

[11] G. Sharma. 2003. "Digital Color Imaging Handbook".

[12] A. McAndrew. "An Introduction to Digital Image Processing with MATLAB", Notes for SCM2511 Image Processing 1, School of Computer Science and Mathematics, Victoria University of Technology.

[13] H. Brettel., F. Viénot., and J. D. Mollon. 1997. "Computerized Simulation of Color Appearance for Dichromats". In Journal of the Optical Society of America A., 14(10), 26472655.

[14] F. Viénot., H. Brettel., L. Ott., B. M’Barek., and J. D. Mollon. 1995. "What do colourblind people see?" In Nature; 376:127-128.

[15] H. Brettel, F. Vienot, and J. Mollon. 1999. "Digital video colourmaps for checking the legibility of displays by dichromats”, In Color Research and Application 24, 243-252.

[16] V.A. Kovalev. 2004. “Towards image retrieval for eight percent of color-blind men”,In 17th International Conference on Pattern Recognition (ICPR'04), PP. 943-946.

[17] G.W. Meyer and D.P. Greenberg. 1988. "Color-defective vision and computer graphics displays", In IEEE Computer Graphics and Applications 8, 28-40.

[18] F. Viénot., H. Brettel., and J. D. Mollon. 1999. "Digital video colourmaps for checking the legibility of displays by dichromats", In Color Res. Application, 24, pp243-252.

[19] S. Kondo. 1990. "A Computer Simulation of Anomalous Color Vision". In Ohta, ed., Color Vision Deficiencies IX, Kugler \& Ghedini, 145-159.

[20] G. Machado, M. Oliveira, and L. Fernandes. 2009. "A Physiologically-based Model for Simulation of Color Vision Deficiency”, In IEEE TVCG, 15(6), 1291-1298.

[21] K. Mancuso., W. W. Hauswirth., Q. Li., T. B. Connor., J. A. Kuchenbecker., M. C. Mauck., J. Neitz and M. Neitz. 2009. "Gene therapy for red-green colour blindness in adult premates”. In Nature. Vol. 461, Issue 7265, p784-787. 
[22] G.F. Muttaqin and I.S. Suwandi. 2011. "Simulation system of color blind glasses by image processing". In Proc. ICEEI, pp.1-4.

[23] C. L. Lai and S. W. Chang. 2009. "An Image Processing Based Visual Compensation System for Vision Defects". In Proceedings of the 2009 Fifth International Conference on Intelligent Information Hiding and Multimedia Signal Processing (IIH-MSP '09). IEEE Computer Society, Washington, DC, USA.

[24] A. Tagarelli., A. Piro., G. Tagarelli., P. B. Lantieri., D. Risso and R. L. Olivieri. 2004. "Colour blindness in everyday life and car driving", In ACTA OPHTHALMOLOGICA SCANDINAVICA; 82(4):436-42.

[25] P. Capilla., M. J. Luque., and M. A. Diez-Ajenjo. 2004. "Looking for the dichromatic version of a colour vision model", In Optical Society of America Journal, Volume 6, pp. 906919.

[26] F. Viénot., and H. Brettel. 2001. "Color display for dichromats", In Proc. SPIE Vol. 4300 pp. 199-207.

[27] P. Capilla., M. A. Diez-Ajenjo., M. J. Luque., and J. Malo. 2004. "Corresponding-pair procedure: a new approach to simulation of dichromatic color perception”, In Optical Society of America Journal, Volume 21, Issue 2, pp. 176-186.

[28] D. McIntyre. 2002. "Colour Blindness: Causes and Effects". Dalton Publishing.

[29] K. Rasche., R. Geist., and J. Westall. 2005. "Detail Preserving Reproduction of Color Images for Monochromats and Dichromats". In IEEE Comput. Graph. Appl. 25, 3 (May 2005), 22-30.

[30] J. B. Huang., Y. C. Tseng., S. I. Wu., and S. J. Wang. 2007. "Information Preserving Color Transformation for Protanopia and Deuteranopia”. In IEEE Signal Processing Letters. 14, 10 (October 2007), 711-714.

[31] K. Rasche., R. Geist., and J. Westall. 2005. "Re-coloring of Images for Gamuts of Lower Dimension”. In Eurographics 2005. 24, 3, 423-432. 
[32] J. Walraven and J.W. Alferdinck. 1997. "Color Displays for the Color Blind", In Proc. IS\&T and SID 5th Color Imaging Conf., Soc. for Imaging Science and Technology, pp. 17-22.

[33] T. Ohkubo and K. Kobayashi. 2008. "A Color Compensation Vision System for Colorblind people”, In SICE Annual Conference, pp. 1286-1289.

[34] J. Birch. 2001. "Diagnosis of Defective Color Vision", Second edition. ButterworthHeinemann, United Kingdom, Edinburgh.

[35] S. Fomins and M. Ozolinsh. 2011. "Multispectral Analysis of Color Vision Deficiency Tests”, In Materials Science / Medziagotyra, 17 Issue 1, p104-108. 5p.

[36] T. Kohonen. 1989. "Self-Organization and Associative Memory", Springer-Verlag Inc., New York, NY, USA.

[37] T. Kohonen. 1979. "Self-Organizing Maps", Springer-Verlag New York, Inc., Secaucus, NJ, USA.

[38] H. Jin, W.H. Shum, K.S. Leung, and M.L. Wong. 2004. "Expanding self-organizing map for data visualization and cluster analysis", In Information Sciences, 163,157-173.

[39] J. Tumblin and H. Rushmeier. 1993. "Tone Reproduction for Realistic Images", In IEEE Computer Graphics and Applications, vol. 13, no. 6, pp. 42-48.

[40] E. J. Stollnitz, V. Ostromoukhov., and D. Salesin. 1998. "Reproducing color images using custom inks”, In Proceedings of ACM SIGGRAPH 1998, pp. 267-274. 3.

[41] J. Neitz and G. Jacobs. 1986. "Polymorphism of the Long-Wavelength Cone in Normal Human Colour Vision”. In Nature, 323, 623-625. 